\title{
Structural variants shape driver combinations and outcomes in pediatric high-grade glioma
}

Frank Dubois ( $\square$ fdubois@broadinstitute.org)

Dana-Farber Cancer Institute https://orcid.org/0000-0002-7654-6208

\section{Frank Dubois}

Dana-Farber Cancer Institute

Ofer Shapira

The Broad Institute of MIT and Harvard, Cambridge, Massachusetts 02124, USA

Noah Greenwald

Stanford https://orcid.org/0000-0002-7836-4379

Travis Zack

Dana Farber Cancer Institute

Jeremiah Wala

Dana-Farber Cancer Institute

Jessica Tsai

Dana-Farber Cancer Institute

Djihad Hadjadj

McGill University

Alexander Crane

Dana-Farber Cancer Institute

Ashot Harutyunyan

McGill University https://orcid.org/0000-0002-9050-9315

Kiran Kumar

Dana-Farber Cancer Institute

Mirjam Blattner-Johnson

German Cancer Research Center

Jayne Vogelzang

Dana-Farber Cancer Institute

Cecila Sousa

Dana-Farber Cancer Institute

Kyung Shin Kang

Dana-Farber Cancer Institute

Claire Sinai

Dana-Farber Cancer Institute

Dayle Wang 
Dana-Farber Cancer Institute

\section{Prasidda Khadka}

Dana-Farber Cancer Institute

\section{Kathleen Lewis}

Broad Institute of MIT and Harvard

\section{Lan Nguyen}

Broad Institute of MIT and Harvard

\section{Hayley Malkin}

Dana-Farber Cancer Institute

\section{Patricia Ho}

Columbia University https://orcid.org/0000-0002-4622-8188

\section{Ryan O'Rourke}

Dana-Farber Cancer Institute

\section{Rose Gold}

Dana-Farber Cancer Institute

\section{Davy Deng}

Dana-Farber Cancer Institute

Jonathan Serrano

NYU Langone Medical Center

\section{Matija Snuderl}

New York University Medical Center

\section{Karen Wright}

Dana-Farber Cancer Institute

\section{Susan Chi}

Boston Children's Hospital, Harvard Medical School

Jacques Grill

Institut Gustave Roussy

\section{Claudia Kleiman}

Jewish General Hospital https://orcid.org/0000-0002-5158-7126

\section{Liliana Goumnerova}

Boston Children's Hospital and Harvard Medical School https://orcid.org/0000-0002-2662-719X

\section{Nada Jabado}

McGill University and The Research Institute of the McGill University Health Centre

https://orcid.org/0000-0003-2485-3692

\section{David Jones}

Hopp Children's Cancer Center Heidelberg (KiTZ) https://orcid.org/0000-0002-2036-5141

\section{Mark Kieran}

Dana-Farber Cancer Institute

\section{Keith Ligon}


Dana-Farber Cancer Institute

\section{Rameen Beroukhim}

Department of Cancer Biology, Dana-Farber Cancer Institute and Harvard Medical School https://orcid.org/0000-0001-6303-3609

\section{Pratiti Bandopadhayay}

Dana-Farber Cancer Institute

\section{Biological Sciences - Article}

Keywords: Pediatric high-grade gliomas (pHGGs), diffuse midline gliomas (DMGs), pediatric high-grade glioma

Posted Date: April 14th, 2021

DOI: https://doi.org/10.21203/rs.3.rs-389596/v1

License: (c) (1) This work is licensed under a Creative Commons Attribution 4.0 International License. Read Full License

Version of Record: A version of this preprint was published at Nature Cancer on July 4th, 2022. See the published version at https://doi.org/10.1038/s43018-022-00403-z. 


\section{Structural variants shape driver combinations and outcomes in pediatric high-grade glioma}

Frank PB Dubois ${ }^{1,2}$, Ofer Shapira ${ }^{1,2}$, Noah F Greenwald ${ }^{1,2}$, Travis Zack ${ }^{1,2}$, Jeremiah Wala ${ }^{1,2}$, Jessica W

4 Tsai $^{2,3,4}$, Djihad Hadjadj ${ }^{5}$, Alexander Crane ${ }^{1,2}$, Ashot S Harutyunyan ${ }^{5}$, Kiran H Kumar ${ }^{1,2}$, Mirjam BlattnerJohnson $^{6}$, Jayne Vogelzang ${ }^{7}$, Cecila Sousa7, Kyung Shin Kang ${ }^{1,2}$, Claire Sinai ${ }^{7}$, Dayle K Wang ${ }^{2,4}$, Prasidda

Khadka $^{1,2}$, Kathleen Lewis ${ }^{2}$, Lan Nguyen², Hayley Malkin7, Patricia Ho ${ }^{1,2}$, Ryan O'Rourke ${ }^{1,2}$, Rose Gold ${ }^{1,2}$, Davy Deng ${ }^{1,2}$, Jonathan Serrano ${ }^{8}$, Matija Snuderl ${ }^{8}$, Karen D Wright ${ }^{3,4}$, Susan N Chi ${ }^{3,4}$, Jacques Grill ${ }^{9}$,

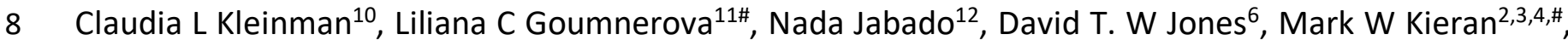
Keith L. Ligon ${ }^{2,7,13 *}$, Rameen Beroukhim ${ }^{1,2,14 *}$, Pratiti Bandopadhayay ${ }^{2,3,4 *}$

${ }^{1}$ Departments of Cancer Biology, Dana-Farber Cancer Institute and Harvard Medical School, Boston, MA, 02215, USA

${ }^{2}$ Broad Institute of MIT and Harvard, Cambridge, MA, 02142, USA

${ }^{3}$ Department of Pediatrics, Harvard Medical School, Boston, MA, 02215, USA

${ }^{4}$ Department of Pediatric Oncology, Dana-Farber Boston Children's Cancer and Blood Disorders Center, Boston, MA, 02215, USA

${ }^{5}$ Department of Human Genetics, McGill University, Montreal, QC H3A 0C7, Canada

${ }^{6}$ Hopp Children's Cancer Center Heidelberg (KiTZ);Pediatric Glioma Research Group, German Cancer Consortium (DKTK), German Cancer Research Center (DKFZ), Heidelberg, Germany.

${ }^{7}$ Department of Oncologic Pathology, Dana-Farber Cancer Institute, Harvard Medical School, Boston, MA, USA

${ }^{8}$ New York University Medical Center, New York, NY 10016, USA.

${ }^{9}$ Department of Pediatric and Adolescent Oncology and INSERM Unit 981, Gustave Roussy Institute and University of Paris Saclay, Villejuif, France

${ }^{10}$ Department of Human Genetics, McGill University, Montreal, QC H3A 0C7, Canada; Lady Davis Research Institute, Jewish General Hospital, Montreal, QC H3T 1E2, Canada

${ }^{11}$ Department of Neurosurgery, Boston Children's Hospital; Dana Farber/Boston Children's Cancer and Blood Disorders Center, Boston, MA, 02215 USA.

${ }^{12}$ Department of Human Genetics, McGill University, Montreal, QC H3A 0C7, Canada; Division of Experimental Medicine, Department of Medicine; Department of Pediatrics, McGill University, and The Research Institute of the McGill University Health Centre, Montreal, QC H4A 3J1, Canada.

${ }^{13}$ Department of Pathology, Brigham \& Women's Hospital, Boston; Center for Patient Derived Models, DanaFarber Cancer Institute; Department of Pathology, Boston Children's Hospital and Harvard Medical School, Boston, MA, 02215, USA

${ }^{14}$ Medical Oncology, Dana-Farber Cancer Institute and Harvard Medical School, Boston, MA, 02215, USA

* Current affiliation: LCG: Tromboprotea, MWK:Bristol-Myers Squibb, Boston, Devens, MA, 01434, USA; and Lawrenceville, NJ, 08648, USA.

*Corresponding authors (these authors jointly supervised this work):

Keith Ligon: keith_ligon@dfci.harvard.edu

Rameen Beroukhim: rameen beroukhim@dfci.harvard.edu

Pratiti Bandopadhayay: pratiti_bandopadhayay@dfci.harvard.edu 


\section{Abstract}

18

20

22

24

26

28

30

32

34

36

38

Pediatric high-grade gliomas (pHGGs), encompassing hemispheric and diffuse midline gliomas (DMGs), remain a devastating disease. The last decade has revealed oncogenic drivers including single nucleotide variants (SNVs) in histones. However, the contribution of structural variants (SVs) to gliomagenesis has not been systematically explored due to limitations in early SV analysis approaches. Using SV algorithms, we recently created, we analyzed SVs in whole-genome sequences of 179 pHGGs including a novel cohort of treatment naïve samples-the largest WGS cohort assembled in adult or pediatric glioma. The most recurrent SVs targeted MYC isoforms and receptor tyrosine kinases, including a novel SV amplifying a MYC enhancer in the IncRNA CCDC26 in 12\% of DMGs and revealing a more central role for MYC in these cancers than previously known. Applying de novo SV signature discovery, we identified five signatures including three (SVsig1-3) involving primarily simple SVs, and two (SVsig45) involving complex, clustered SVs. These SV signatures associated with genetic variants that differed from what was observed for SV signatures in other cancers, suggesting different links to underlying biology. Tumors with simple SV signatures were TP53 wild-type but were enriched with alterations in TP53 pathway members PPM1D and MDM4. Complex signatures were associated with direct aberrations in TP53, CDKN2A, and RB1 early in tumor evolution, and with extrachromosomal amplicons that likely occurred later. All pHGGs exhibited at least one simple SV signature but complex SV signatures were primarily restricted to subsets of $\mathrm{H} 3.3^{\mathrm{K} 27 \mathrm{M}} \mathrm{DMGs}$ and hemispheric pHGGs. Importantly, DMGs with the complex SV signatures SVsig4-5 were associated with shorter overall survival independent of histone type and TP53 status. These data inform the role and impact of SVs in gliomagenesis and mechanisms that shape them. 


\section{Introduction}

Pediatric high-grade gliomas (pHGGs), encompassing diffuse midline gliomas (DMGs) and hemispheric tumors, represent the most common cause of cancer-related deaths in children age 0-14 years ${ }^{1}$. Over the last decade, several landmark papers have revealed recurrent single nucleotide variants (SNVs) in the core histones H3.3 and $\mathrm{H} 3.1$ including $\mathrm{H} 3.1^{\mathrm{K} 27 \mathrm{M}}, \mathrm{H} 3.3^{\mathrm{K} 27 \mathrm{M}}$, and $\mathrm{H} 3.3^{\mathrm{G} 34 \mathrm{R}}$ mutations, co-occurring with alterations in the TP53 signaling pathway and receptor tyrosine kinases $(\mathrm{RTKs})^{2-9}$. Such information has been generally derived from exome or targeted sequencing panels, limiting the ability to characterize structural variants (SVs). As such the role of such events in pHGG is underexplored.

SVs represent connections, or rearrangements, between distant genomic loci and are critical drivers in other cancers. They underlie all somatic copy-number alterations (SCNAs) except whole chromosome gains and losses, thereby altering more of the genome than any other genetic alteration. A single SV can have long-range effects on dozens to hundreds of genes. In some cases, SVs result in extrachromosomal amplifications (otherwise known as double minutes $)^{10}$ that can lead to hundreds of copies of an oncogene per cell ${ }^{11-15}$. Moreover, the effects of SVs on cellular fitness often result from changes in chromatin structure such as disruption of topologically associated domains (TADs) and gene-enhancer interactions ${ }^{16-21}$. Therefore, unlike SNVs, the SVs with the largest effects on selection are often outside of the exome and require whole-genome sequencing (WGS) for their characterization. Moreover, these distant effects raise challenges to interpreting the effects of individual SVs.

Both the frequency with which SVs recur at individual loci, and the mechanisms by which they are generated, can vary widely across cancers. Recently, we and others have developed analytic tools to detect both the recurrent, likely oncogenic SVs, and patterns of SVs (SV signatures). These signatures can indicate the mechanisms by which SVs are generated ${ }^{22-26}$, and recent efforts have begun to characterize them in breast ${ }^{26}$, ovarian ${ }^{27,28}$ and other cancer types ${ }^{23}$. However, unlike SNVs, whose signatures have been characterized across tens of thousands of exomes, no consensus catalog of SV signatures exists, and the relationships between currently described SV signatures and other cancer types has not been well-explored. For example, high rates of tandem duplications have been associated with deficiencies in homologous recombination (HR) only in tumors with very high SV burdens ${ }^{23,29-31}$. It remains unclear if these associations translate to other tumor types, including pHGGs, and which other SV signatures and associated variant-generating processes exist.

The differences across lineages indicate a substantial role for epigenetics in shaping the SVs that are observed in cancer $^{22,23}$. Mutations in core histones were first reported in $\mathrm{pHGGs}^{8,9}$, highlighting the role of epigenetic dysregulation in these tumors. pHGGs therefore offer a unique perspective on the relationships between patterns of SVs and different alterations in chromatin. Associations between patterns of SVs and other molecular and clinical characteristics of these tumors are also largely unknown.

One obstacle to the genomic characterization of DMGs has been a lack of pre-treatment tissue. Due to their location, most often in the pons, biopsies were traditionally avoided. A concern in characterizing post-treatment samples is that treatment-most often involving ionizing radiation-might itself alter the SV patterns detected in these tumors. Recently, however, pre-treatment biopsies have been performed more widely due to landmark clinical trials showing their safety ${ }^{32-35}$.

We leveraged pre-treatment biopsies from these studies, as well as novel and published data from both pretreatment biopsy and autopsy samples of DMGs and hemispheric tumors, to assemble the largest WGS cohort to 


\section{Results}

90

92

date from pHGGs. We then applied recently developed tools to sensitively detect SVs, identify recurrent and likely driver events, and stratify pHGGs based upon mechanistically informative SV signatures. We further detected genetic events and differences in clinical outcome associated with patterns of SVs across the genome.

\section{Significantly recurrent SVs}

In order to characterize SVs in pHGG including samples whose genomes had not been altered by treatment, we assembled a cohort of pHGG WGS data from 179 children including 61 hemispheric tumors and 118 diffuse midline gliomas, or DMGs. Of these, 61 are novel to this study (Supp. Table 1). Among the DMGs, 84 (71\%) were from pretreatment biopsies including 33 obtained from the first multi-institutional North American clinical trial to incorporate diagnostic biopsies ${ }^{35}$. The tumor purity of the pre-treatment biopsies was comparable to autopsy samples (median: 0.8 vs. $0.78, p=0.5$ ) (Ext. Data Fig. 1A). This represents the largest cohort of pretreatment DMGs with whole genome sequencing to date.

In analyses of somatic copy-number alterations (SCNAs), we were able to detect a new recurrent amplification in 8q24.21, 2 MB telomeric to MYC. In total, 28 tumors (16\%) exhibited absolute copy-numbers (CNs) > 3.7. In more than half of these (15/28), the amplicon excluded MYC itself, such that they defined a peak region of amplification that did not encompass MYC (Ext. Data Fig. 1C). All but one of these were DMGs, representing a significant enrichment $(p=0.0016)$. A separate peak was also detected that did encompass MYC without the telomeric region, due to two tumors with extrachromosomal amplicons that linked MYC to other loci. The new amplification peak may not have been detected in prior array- and exome-based studies because it encompasses only $200 \mathrm{~kb}$ and lies entirely outside of the exome ${ }^{3}$. Aside from this amplicon, the results of SCNA and SNV recurrence analyses largely agreed with those detected in prior studies ${ }^{3-7}$. These include high frequencies of $\mathrm{H} 3.3^{\mathrm{K} 27 \mathrm{M}}(50 \%)$ or $\mathrm{H} 3.1^{\mathrm{K} 27 \mathrm{M}}$ (12\%) mutations, reflecting the enrichment for midline glioma in this study, and other known drivers of pHGG (Ext. Data Fig. 1B).

We also made the novel finding that several previously-described regions were recurrently amplified together to high levels, such as 2p25.1 and the MYCN locus at 2p24.3. This pattern of apparently linked SCNAs in distinct genomic loci suggests underlying recurrent SVs. We therefore comprehensively catalogued SVs using an assemblybased method $^{36}$ that is capable of detecting complex and short SVs that are not easily detected using standard alignment-based methods. In total, we detected 15,485 SVs, averaging 87 per tumor, including 1482 (10\%) that were 10-300 bp in span, a "blind spot" in prior analyses ${ }^{36}$.

To distinguish recurrent SVs, we took two approaches based upon methods that we recently developed ${ }^{37}$. In the first, we conducted a "1D" analysis to detect loci with significantly recurrent SV breakpoints (SRBs). In the second, we conducted a "2D" analysis to detect pairs of loci that are recurrently juxtaposed by SVs ("significantly recurrent juxtapositions", or SRJs). SRBs might not appear within SRJs if they connect to a dispersed set of loci. Conversely, breakpoints within SRJs may not be SRBs; the finding of recurrent juxtapositions between two loci increases our sensitivity for SRJs whose individual breakpoints might be insufficiently frequent to be noted as SRBs. 
We identified SRBs in five TADs (Supp. Table 3 or Fig. 1A, and Ext. Data Fig. 1D) and two SRJs (Fig. 2A; Ext. Data

128

130

132

134

136

138

140

142

144

146

148

150

152

154

156

158

160

162

164

166

168

170

Fig. 3A). The most significant SRB was within the MYC TAD, in the long non-coding RNA (IncRNA) CCDC26. The same locus was also identified as a component of an SRJ connecting two loci at the telomeric end of the MYC TAD. The remaining SRBs corresponded to SVs within the TADs of the receptor tyrosine kinases (RTKs) MET ( $q=0.0025)$, EGFR ( $q=0.029$ ), and PDGFRA ( $q=0.032$ ), as well as an SV within the TAD of the transcription factor ID2. This latter SRB was also a component of the second SRJ, which connected the loci of ID2 and MYCN. We describe each of these in more detail below.

\section{Rearrangements within the InCRNA CCDC26 within the MYC TAD activate MYC by amplifying an interacting enhancer}

Our 1D and 2D analyses both nominated rearrangements within the MYC TAD as among the most significantly recurrent SVs in pHGG. A total of 28 SVs were identified within this TAD. Among these, six were complex rearrangements that amplified the MYC protein coding sequence, connecting the amplicon to locations outside of the MYC TAD. An additional seven rearrangements were dispersed deletions or low-quality SV calls, which we classified as potential passenger events or artifacts. The remaining 15 represented a tandem duplication centering on intron 1 of the IncRNA CCDC26, with a median span of $216 \mathrm{kbp}$ and a minimal common region of amplification (MCR) of only $42 \mathrm{~kb}$ (Fig. 1B).

The $2 \mathrm{Mb}$ region telomeric to MYC has been shown to contain MYC enhancers in hematopoietic lineages ${ }^{38}{ }_{-}^{39}$, neuroblastoma ${ }^{40}$, and lung and endometrial carcinomas ${ }^{41}$, but with lineage specific enhancer locations ${ }^{38,41}$. We therefore hypothesized that the CCDC26 amplicon promotes oncogenesis by amplifying an associated enhancer. We analyzed previously published $\mathrm{H} 3 \mathrm{~K} 27$ ac enhancer tracks generated from $\mathrm{H}^{\mathrm{K} 27 \mathrm{M}}$ mutant and wild-type $\mathrm{pHGGs}^{42}$ and adult glioblastomas ${ }^{43}$ and observed H3K27ac enhancer peaks within the MCR of the CCDC26 amplicon (Figure 1C, Ext. Data Fig. 2A). We also validated the presence of the CCDC26 associated enhancer in an independent dataset that leveraged ATAC-seq to profile the chromatin landscape of an independent cohort of $\mathrm{pHGGs}^{44}$. This enhancer also appears to be present in normal neural tissue available through ENCODE ${ }^{45,46}$. In contrast, enhancer maps from cells derived from hematopoietic and lung tissues did not show peaks at this location (Ext. Data Fig. 2A). We conclude that the CCDC26 amplicon centers on a neural lineage-specific enhancer peak.

Although this enhancer is present across neural tissues, histone mutant DMGs exhibit 31\% more H3K27ac binding at this locus than $\mathrm{H}^{\mathrm{WT}}$ gliomas, constituting the fourth most differential super enhancer between these groups ( $q$-value $=0.05 ; p=0.001)^{42}$. Intriguingly, the CCDC26 amplicon within our dataset also occurred almost exclusively in $\mathrm{H}^{\mathrm{K} 27 \mathrm{M}}$ gliomas (present in $14 / 97 \mathrm{H} 3^{\mathrm{K} 27 \mathrm{M}}$ vs. $1 / 82 \mathrm{H} 3^{\mathrm{G} 34 \mathrm{R}}$ mutant or $\mathrm{H} 3^{\mathrm{WT}}$ tumors; $\mathrm{q}=0.0018$ ). These data indicate that the SRB in CCDC26 amplifies an enhancer which is present in the whole neural lineage but shows a quantitative enrichment specifically in $\mathrm{H}^{\mathrm{K} 27 \mathrm{M}}$ gliomas.

This lineage-specific enhancer also appears to interact directly with MYC. We interrogated Hi-C data from an $\mathrm{H} 3.3^{\mathrm{K} 27 \mathrm{M}}$ mutant $\mathrm{PHGG}$, two patient-derived $\mathrm{H} 3.3^{\mathrm{K} 27 \mathrm{M}}$ mutant cell lines, and iPSC-derived neural progenitors, and found a prominent interaction peak between the MCR of the CCDC26 amplicon and the MYC promoter in all of them (Fig. 1D; Ext. Data Fig. 2B). We also observed increased MYC expression in $\mathrm{pHGGs}$ with this amplicon relative to samples without any SCNAs in the MYC TAD (Fig. 1E; $p=0.04$ ), supporting the hypothesis that the amplicon targets a MYC enhancer. Moreover, these CCDC26-SV samples had similar MYC expression as samples with amplicons encompassing the MYC coding region $(p=0.85)$. To functionally validate enhancer activity of the 
H3K27ac peak in the CCDC26 amplicon, we generated two enhancer reporter systems (E1 and E2), each encompassing slightly more than one half of the overall enhancer sequence, with a small region of overlap. The E1 subset showed significant enhancer activity ( $p=0.019, n=3$, T-test) compared to the reporter construct without insert. (Fig. 1F-G).

These results indicate that the most significantly recurrent SV in histone mutant pHGGs amplifies a neural-lineage MYC enhancer that is augmented in the $\mathrm{H} 3^{\mathrm{K} 27 \mathrm{M}}$ context, thereby increasing MYC expression.

\section{Activation of MYCN through enhancer amplification and hijacking}

182

184

186

188

190

192

194

Somatic enhancer amplification also seemed to play a role in the activation of MYCN in PHGG. The SRJ connecting ID2 with MYCN represents a set of highly complex SVs in tumors with high-level amplifications within this region on chromosome 2 (Fig. 2B). ID2 is a transcription factor that is well-recognized to play essential roles in regulating neural differentiation ${ }^{47,48}$. Accordingly, we observe the ID2 locus to be associated with an H3K27ac enhancer that is present across all analyzed pHGG tumor samples (Ext. Data Fig. 3B). The MCR of the MYCN-ID2 amplification contains both the ID2-associated enhancer peak and the coding sequence of MYCN (Ext. Data Fig. 3B). The SVs result in juxtaposition of the enhancer in ID2 with $M Y C N$, reducing the distance between the two from the normal $7 \mathrm{Mbp}$ to less than $700 \mathrm{kbp}$ (Fig. 2C). These data suggest these SVs hijack the ID2 enhancer to activate MYCN.

We also identified four pHGGs with $M Y C N$ amplifications that did not connect to ID2. However, these latter "localized MYCN" amplicons always encompassed more of the immediate neighborhood to MYCN than the complex MYCN-ID2 amplicons above. In contrast to MYCN-ID2 amplicons, which only contained a small fraction of the MYCN TAD (23\% on average), localized MYCN amplicons contained most of this TAD (60\% on average, $\mathrm{p}=0.03$, T-test), including several enhancers that are not in the MYCN-ID2 amplicons (Fig. 2F).

The high-level MYCN amplicons show typical characteristics of extrachromosomal amplicons (EC-amps), reaching copy numbers of $50-300$ per cell. Other oncogenes with absolute copy numbers of more than 10 have been shown to be amplified on double minutes in various cancer types ${ }^{14,49}$. While projections on the linear reference genome resulted in complex patterns that are typical of EC-amps ${ }^{15,50}$, it was possible to construct simple circular amplicons containing MYCN and, in the MYCN-ID2 cases, ID2 (Fig. 2D). Indeed, these circular amplicons represent an optimal solution to explain the joint copy number and SV profiles in this region. The ID2-MYCN amplicons appeared to reflect multiple subclonal amplicons including some containing additional oncogenes such as MDM4 (Fig. 2E). In contrast, pHGGs with localized MYCN amplicons showed significantly fewer SVs between the MYCN TAD and other TADs (Fig. 2G and Ext. Data Fig. 3C).

We conclude that $M Y C N-I D 2$ rearrangements are likely to be an example of enhancer hijacking, bringing a strong enhancer in ID2 next to MYCN on amplicons without the endogenous elements of the MYCN TAD, whereas amplifications of MYCN without ID2 also amplify enhancers within the MYCN TAD.

Significantly recurrent breakpoints around RTKs also suggest extrachromosomal amplicons

The remaining SRBs all involved receptor tyrosine kinases (RTKS) that are known to be amplified and oncogenic in pHGG: PDGFRA, EGFR, and MET $T^{3-7,51}$. These loci, along with MYC and MYCN, were also the only regions with high- 
level amplifications recurrent in at least three patients (Fig. 3A). Similar to our findings from the MYC isoforms, the RTK SRBs also showed both simple-SVs that presumably amplify local enhancers (Fig. 3B, Ext. Data Fig. 4C) and SVs that appeared to reflect complex extrachromosomal amplicons that integrate distant sites and reach as many as 200 copies (Fig. 3 C and E)

222

Overall, 35/179 tumors showed at least one amplicon (>50 kbp in size) with an absolute copy number greater than ten. Among 34 of these 35 tumors, the high-level amplicons contained at least one well-known oncogene, and apart from the coding sequence of the oncogene, they recurrently incorporated the same genomic loci around the oncogene (exemplified in Ext. Data Fig. 4A-B for PDGFR and EGFR). The high-level amplicons in several pHGGs also contained multiple oncogenes from distant locations (Fig. 3D-E). In some cases, we detected SVs connecting these oncogenes with supporting reads that allowed for the reconstruction of circular extrachromosomal amplicons containing oncogenes from different chromosomes (Fig. 3E). This might suggest either synchronous chromothripsis of two chromosomes or translocation of one oncogene next to another with subsequent circularization. These data suggest that only a subset of pHGGs develops high-level amplicons, which recurrently contain the same (presumably regulatory) sequences in addition to the target oncogene.

To further understand the structure of these amplicons, we first focused on high-level amplicons containing PDGFRA. These amplicons span more than $2.5 \mathrm{Mbp}$ and are super-imposed upon low-level amplicons of the surrounding region, often starting from the centromere. The amplicons included $K I T$ in $80 \%$ of cases and reached KDR in $60 \%$. All but one $(14 / 15,93 \%)$ of amplicons in the PDGFRA TAD amplified the PDGFRA coding sequence itself, often to the highest copy numbers reached in the region (Ext. Data Fig. 4A). The sole exception harbored amplification of a short sequence centromeric to PDGFRA that contains H3K27ac enhancers (Fig. 3B) that have been shown to interact with the PDGFRA promoter in $\mathrm{pHGGs}^{19}$ and adult GBMs, ${ }^{52}$ suggesting use of enhancer amplification to activate PDGFRA. Indeed, this region was included in the PDGFRA amplicon in nearly all tumors (14/15, 93\%) (Ext. Data Fig. 4A). The exception appeared to contain an in-frame fusion of NMU and PDGFRA, suggesting an alternative mode of PDGFRA activation. These data suggest that SVs in pHGG recurrently incorporate an upstream enhancer-rich region into high-level PDGFRA amplicons.

The high-level EGFR and MET amplicons also extended beyond the RTK coding sequence to recurrently involve associated enhancer elements (Ext. Data Fig. 4B-D). The EGFR amplicons showed a clear skew towards the enhancer region in SEC61G, which drives EGFR expression in extrachromosomal amplicons in adult GBM ${ }^{21}$. Both EGFR and MET amplicons showed subclonal SVs within the coding sequence, potentially allowing for expression of alternate transcripts. In two tumors, these resulted in the EGFRvIII variant (one is exemplified in Fig. 3E). Similar to the MYCN-ID2 SVs, EGFR amplicons tended to have a highly complex subclonal structure with incorporation of distant oncogenes (Fig. 3E). MET amplicons also skewed towards a region including enhancer elements in CAPZA2 (Ext. Data Fig. 4D). However, MET amplicons exhibited less subclonal diversity, with simpler circular amplicons containing only MET and its associated enhancers. We conclude that the RTK amplicons are shaped by the epigenetic machinery necessary to drive expression of these oncogenes.

\section{SV signatures relate to genetic and epigenetic tumor states}

To identify the mechanisms underlying the SVs in these tumors, we applied a Bayesian NMF-based analysis ${ }^{24}$ to detect SV signatures, based upon their spans, complexity (abundance of neighboring SVs), and rearrangement type (deletion, duplication or inversion). We identified only five such signatures (Fig. 4A), broadly corresponding 
to simple short ( $<30 \mathrm{kbp}$ ) duplications (SVsig1: "Simple Short Dups"); mixed simple-SVs (all sizes and types except small duplications, SVsig2: "mixed simple"); simple interchromosomal rearrangements (SVsig3: "Simple Interchromosomal"); complex interchromosomal events (SVsig4: "Complex Interchrom.") and complex large SVs (>=1 Mbp, all types, SVsig5: "Complex Large").

We next looked for possible causes and consequences of the pHGG SVsigs signatures by testing for associations between the activity of each SVsig and presence of recurrent and known oncogenic ${ }^{53}$ genetic events (Supp. Table 2). The Complex-SV signatures (SVsigs4-5) were most closely associated with TP53 disruption and oncogenic amplifications encompassing MYC, MYCN, PDGFRA, and EGFR - which often themselves represented complex-SVs - and losses of $C D K N 2 A / B$ and PTEN. These signatures were also anticorrelated with oncogenic mutations in PPM1D and ACVR1 (Supp. Table 4 and Fig. 4B). Simple Interchrom. was associated with TERT promoter mutations. Notably, we observed complex-SV signatures in pHGGs with high SV counts (rho $=0.68, \mathrm{p}<2.2 \mathrm{e}-16$, Spearman's) and disrupted DNA damage response (DDR), and found simple tandem duplications to be enriched in pHGGs with lower SV counts. This contrasts with prior results from several adult cancers, where tandem duplications were

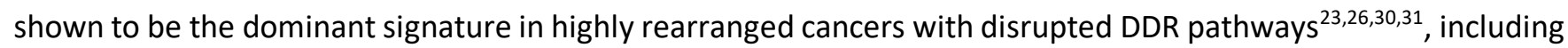
homologous recombination (HR)/BRCA ${ }^{23,54}$. Indeed, none of the genes previously implicated in tandem duplication signatures or loss of HR in adults ${ }^{23,54}$ reached a significant level of association with any signature in this cohort.

The complexity of SVsigs appeared to relate to the epigenetic status of the tumors in which they occurred, and specifically which histone underwent modification. $\mathrm{H} 3.1^{\mathrm{K} 27 \mathrm{M}}$ tumors almost exclusively exhibited the simple SVsigs (SVsigs1-3), harboring significantly fewer gliomas with SVsigs4-5 than H3.3 ${ }^{\mathrm{K} 27 \mathrm{M}}$ tumors $\left(\mathrm{q}=10^{-4}\right)$. H3.3 $3^{\mathrm{G} 34 \mathrm{R}}$ pHGGs tended to exhibit the complex SVsigs while histone wild-type pHGGs had varying SV complexity, but these did not rise to statistically significant levels of enrichment.

Taken together, we conclude that SV signatures associate with distinct genetic variants in pHGG.

288

\section{Associations between SV and SNV signatures}

We next asked whether pHGGs separate into subsets with different DNA damage and damage response characteristics, based on patterns of both SVs and SNVs. Evaluation of SNVs in isolation revealed 14 SNV signatures, including signatures similar to known aging, APOBEC, HR-deficiency and hypermutation SNV signatures $^{25}$ (Supp. Figs. 1-3). Two of these SNV signatures were correlated with SV signatures (Fig. 5A). An SNV signature resembling COSMIC signature 13 (SBS13), thought to be caused by APOBEC mutagenesis especially in

single-stranded DNA around replication forks and sites of DNA damage and repair ${ }^{55,56}$, was associated with Complex-SV signatures ( $\mathrm{q}=2 \times 10^{-16}$ for Complex Large and $10^{-13}$ for Complex Interchrom. signatures). A second SNV signature, resembling COSMIC signature SBS3, was most closely associated with the Mixed Simple-SV signature $\left(q=10^{-13}\right)$ and also with Complex-SV signatures $\left(q=2 \times 10^{-7}\right.$ for Complex Large and $10^{-8}$ for Complex Interchrom.). SBS3 has been associated with HR deficiency ${ }^{25}$.

The entire pHGG cohort separated into three groups reflecting different amplitudes of the five SV and 14 SNV signatures (Fig. 5B-C). The largest cluster ("Complex-SV") was dominated by Complex-SV signatures $\left(\mathrm{q}<4.1 \times 10^{-20}\right.$,

Fig. 4C) and was enriched for TP53 inactivation (q<0.1, Fig 5D), high-level RTK and MYC/MYCN amplicons ( $q<0.2)$ and depleted for $A C V R 1, P P M 1 D$, and $H 3.1^{K 27 M}$ mutations ( $q<0.1$ ). In contrast, the second largest cluster ("SimpleSV") was dominated by the Simple-SV signatures ( $q=0.03$ ), lacked TP53 disruption $(q<0.1)$, and was enriched for 
PPM1D mutations $(\mathrm{q}<0.1$ ). This cluster also contained the hypermutant samples with their distinct SNV signatures,

lacked most SCNAs, and seemed to be driven instead by a combination of SNVs including ACVR1, PPM1D, H3.1 $1^{\mathrm{K} 27 \mathrm{M}}$, and PIK3CA mutations. The smallest cluster ("Intermediate") was enriched with the HR-deficiency SNV signature SBS3 $\left(q=6.9 \times 10^{-5}\right)$ and the Mixed Simple-SV signature $\left(q=4.1 \times 10^{-7}\right)$ and harbored an intermediate number of SVs compared to the other two clusters (Fig. 5E). Despite being TP53 mutant in many cases, tumors in this cluster showed fewer copy-number variants overall with the exception of high-level PDGFRA amplifications. All three clusters included both hemispheric and midline gliomas, although the Intermediate cluster was modestly enriched for the latter ( $q=0.13$, Supplementary Table 1 ). These data suggest that distinct variant generating processes shape pHGG genomes.

\section{Genomic contexts of recurrent SVs and SV signatures}

Different histone mutations are known to be associated with distinct recurrent SNVs and SCNAs; we confirmed these known relationships and expanded them to include two novel associations with SVs ${ }^{3,57}$ (Fig. 6A and B). The significantly recurrent SV in CCDC26 resulting in MYC enhancer amplification was enriched in $\mathrm{H} 3.3^{\mathrm{K} 27 \mathrm{M}}$ gliomas (q $=0.008$ ) and $\mathrm{H} 3.1^{\mathrm{K} 27 \mathrm{M}} \mathrm{pHGGs}$ were enriched for a focal deletion of $C D K N 2 C$ with breakpoints in the adjacent gene $\operatorname{FAF1}(\mathrm{q}=0.04)$.

We also found that H3.3 ${ }^{\mathrm{K} 2 \mathrm{M}}$ pHGGs divided into two groups based on SV complexity. Most exhibited high Complex-SV signature activity, but $42 \%$ did not. These two $\mathrm{H} 3.3^{\mathrm{K} 27 \mathrm{M}}$ groups also exhibited distinct combinations of genetic alterations in known cancer-related genes. We evaluated the statistical significance of these differences by calculating Jaccard distances between genetic profiles across 369 variants in genes from the Cancer Gene Census $^{53}$ for every pair of tumors. H3.3 ${ }^{\mathrm{K} 27 \mathrm{M}}$ Simple-SV pHGGs exhibited short distances from each other, as did H3.3 ${ }^{\mathrm{K} 27 \mathrm{M}}$ Complex-SV pHGGs; however, the distances between the two groups was much greater $\left(\mathrm{q}<6 \times 10^{-8}\right.$; Fig. 5C). Indeed, these H3.3 ${ }^{\mathrm{K} 27 \mathrm{M}}$ Simple-SV pHGGs were as different from the H3.3 ${ }^{\mathrm{K} 27 \mathrm{M}}$ Complex-SV pHGGs as the H3.1 ${ }^{\mathrm{K} 27 \mathrm{M}}$ Simple-SV pHGGs were (Ext. Data Fig. 5A). The H3.3 ${ }^{\mathrm{K} 27 \mathrm{M}}$ Simple-SV pHGGs showed a variant pattern resembling H3.1 ${ }^{\mathrm{K} 27 \mathrm{M}}$ DMGs (Ext. Data Fig. 5B). Mutations in PPM1D, ACVR1, and PIK3CA, gains of 1q encompassing MDM4, and amplifications in CCDC26, encompassing MYC enhancers, were enriched in these tumors relative to

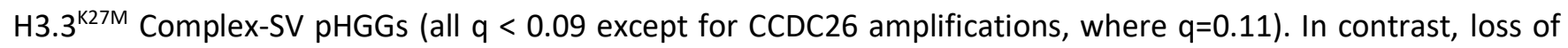
TP53 (by SNV or SCNA) and amplifications of PDGFRA and MYC were depleted (all $\mathrm{q}<0.05$ ). These data suggest that the propensity of pHGGs to develop Complex-SVs influences the combination of driver alterations they accrue, even within groups defined by their histone mutations.

Across the DMGs with more than 20\% Complex-SV signature activity ("H3 ${ }^{\mathrm{K} 27 \mathrm{M}}$ Complex-SV", including H3.3 $3^{\mathrm{K} 27 \mathrm{M}}$ $342[\mathrm{n}=43]$ and $\left.\mathrm{H} 3.1^{\mathrm{K} 27 \mathrm{M}}[\mathrm{n}=3] \mathrm{DMGs}\right)$, the TP53 pathway was inactivated almost universally through direct disruption of TP53 (44/46 cases, 96\%; Fig 6A). In contrast, the majority of DMGs with less complex signature activity ("H3 ${ }^{\mathrm{K} 27 \mathrm{M}}$ 344 simple-SV", H3.3 ${ }^{\mathrm{K} 27 \mathrm{M}}[\mathrm{n}=30]$ and H3.1 $\left.{ }^{\mathrm{K} 27 \mathrm{M}}[\mathrm{n}=21]\right)$ lacked such direct TP53 disruption (37/51, 73\% TP53 ${ }^{\mathrm{WT}} ; \mathrm{q}=$ $\left.2.3 \times 10^{-5}\right)$, but appeared to suppress the TP53 pathway through other mechanisms. Mutations in PPM1D were 346 more prevalent in this group, though still in a minority $\left(7 / 30 \mathrm{H3} .3^{\mathrm{K} 27 \mathrm{M}}, 2 / 21 \mathrm{H3} .1^{\mathrm{K} 27 \mathrm{M}}, 20 \%\right.$ in total; vs $1 / 46 \mathrm{H} 3^{\mathrm{K} 27 \mathrm{M}}$ complex tumors; $q=0.008$ ). It is possible that gains of $1 q$, encompassing MDM4, also served to suppress the TP53 pathway in these tumors. Although 1q spans approximately 2580 genes, we observed two sources of evidence that their prevalence in Simple-SV DMGs related to MDM4 and TP53 pathway suppression. First, MDM4 was these 1q gains were the only arm-level SCNAs that anti-correlated with disruption of TP53 $\left(q=2.8 \times 10^{-6}\right.$ in $\mathrm{H}^{\mathrm{K} 27 \mathrm{M}}$ - 
DMGs; $q=0.0003$ across all pHGGs, Ext. Data Fig. 5C-D) apart from gains of chromosome $2(q<0.0025)$. In contrast, seven of the other thirteen significantly recurrent arm-level SCNAs were positively correlated with TP53 disruption in $\mathrm{H}^{\mathrm{K} 27 \mathrm{M}}$-DMGs (all $\mathrm{q}<0.018$ ), presumably due to the role of TP53 in generating aneuploidies ${ }^{58,59}$. Indeed, across $\mathrm{TP} 3^{\mathrm{WT}} \mathrm{H} 3^{\mathrm{K} 27 \mathrm{M}} \mathrm{DMGs}$, gains of $1 \mathrm{q}$ were among the most common genetic events, observed in $85 \%$ of tumors $(16 / 20$ $\left.\mathrm{H} 3.3^{\mathrm{K} 27 \mathrm{M}}, 17 / 19 \mathrm{H3} .1^{\mathrm{K} 27 \mathrm{M}}\right)$, as opposed to $31 \%$ of TP53-disrupted H3 ${ }^{\mathrm{K} 27 \mathrm{M}}$ DMGs $\left(\mathrm{p}=1.4 \times 10^{-6}\right)$. No pHGGs in our cohort exhibited focal, high-level amplifications of MDM2. These data suggest that direct disruption of TP53 contributes to a different pattern of SVs compared to other mechanisms of TP53 pathway inactivation, which occur primarily through alterations of PPM1D and MDM4.

Focusing on the tumors that harbored significantly recurrent SVS (SRSVs), we observed two groups. One group contained the tumors with high-level amplicons of the coding sequences of the oncogenes PDGFRA, EGFR, MET, $M Y C$, and MYCN ("Onc-amp"). In contrast, the second group amplified presumed enhancer elements within the TADs of these oncogenes without amplifying their coding sequences ("TAD-amp"). The Onc-amp pHGGs exhibited significantly higher activity of the Complex-SV signatures $\left(p=4 \times 10^{-7} ; \mathrm{Fig}\right.$. 7A-B). Intriguingly, the two groups also

harbored inactivating alterations in different DNA damage response genes (Fig. 7A). Onc-amp pHGGs were enriched for TP53 SNVs (69\% of Onc-amp vs. $18 \%$ of TAD-amp pHGGs, $\mathrm{q}=0.01$ ) and RB1 deletions (23\% of Oncamp vs. $0 \%$ of TAD-amp pHGGs, $q=0.16)$. In contrast, TAD-amp pHGGs were enriched with PPM1D SNVs ( $29 \%$ of TAD-amp vs. $0 \%$ of Onc-amp pHGGs, q=0.03) and gains of $1 q$ encompassing MDM4 (71\% of TAD-amp vs. $34 \%$ of Onc-amp pHGGs, $\mathrm{q}=0.16)$. In sum, alterations in TP53 and RB1 associate with Complex-SV signatures and highlevel amplifications of oncogene coding sequences, while PPM1D SNVs and 1q gains more frequently occur with Simple-SV signatures and amplifications of enhancer elements near oncogenes.

\section{Temporal evolution of genetic variants}

These data raise the possibility that alterations in DNA-damage responses (DDR) not only shape the processes that generate SVs but also the types of driver alterations they exhibit in MYC, MYCN and RTKs. Specifically, we considered two hypotheses in regards to tumor evolution. First, disruption of DDR could be an early event that activates the Complex-SV generating process and culminates in the development of a specific class of genetic events including the high-level RTK and MYCN amplicons described earlier. Alternatively, both disruption of the DDR and the high-level oncogene amplification could happen later in tumor development as a consequence of the complex SVs involving these cancer genes.

Notably, we observed no effects of therapy on SV patterns, suggesting that the SVs occurred during gliomagenesis. Although radiation treatment has been shown to induce DNA breaks ${ }^{60}$, we found no differences in the number of SVs per sample (median 35 vs $42 ; q=0.6$ ) or in the activity of the Complex-SV signatures (median $24 \%$ vs $28 \%$; $\mathrm{q}=0.7$ ) between pre-treatment biopsy and autopsy samples (Supp. Figs. 4A-B).

We therefore performed a timing analysis reflecting the relative ordering of mutations and SCNAs during gliomagenesis ${ }^{61}$. We found that the focal amplification of the MYC enhancer in CCDC26 is one of the earliest variants in the TAD-amp samples (Fig. 7C), occurring earlier than alterations in PPM1D and 1q/MDM4 gain. In contrast, the amplification of the oncogenes' protein coding sequences in the Onc-amp samples happened after the loss of the tumor suppressor genes TP53, RB1 and CDKN2A/B (Fig. 7D). These data suggest that simple tandem duplications can arise in tumors without major disruptions of DDR, potentially contributing to tumor initiation, 
whereas the creation of the complex high-level amplicons of MYC isoforms and RTKs requires prior direct genetic disruption of TP53, RB1, or CDKN2A/B.

398

Prior studies have shown histone mutations to be the initiating event in the pHGGs in which they occur $8,9,42,51,62-$

${ }^{64}$. However, the studies investigating pHGG evolution in human tumor tissues were limited to exomic alterations in fewer than 15 patients ${ }^{65-68}$. Our cohort of whole genome sequencing data from 179 patients increased our 402 power to detect differences in timing of genetic events within each histone subgroup. Among SNVs, we confirmed the findings of these previous studies ${ }^{65-68}$ including that $\mathrm{H}^{\mathrm{K} 27 \mathrm{M}}$ mutations are the earliest mutations, followed by 404 SNVs in ACVR1 and TP53 in H3.1 $1^{\mathrm{K} 27 \mathrm{M}}$ and H3.3 $3^{\mathrm{K} 27 \mathrm{M}}$ gliomas, respectively. (Ext. Data Fig. 6). This large cohort of WGS samples also provided an unprecedented opportunity to time focal SCNAs based on the ratio of SNVs acquired 406 before and after each change in each copy number ${ }^{61}$. We found that losses of TP53, CDKN2A/B, and RB1 precede RTK amplifications across each of the H3.3 $3^{\mathrm{K} 27 \mathrm{M}}, \mathrm{H} 3.3^{\mathrm{G} 34 \mathrm{R}}$ and $\mathrm{H} 3^{\mathrm{WT}}$ subgroups of pHGG. In H3.3 $3^{\mathrm{K} 27 \mathrm{M}}$ DMGs, the 408 simple amplifications of the MYC enhancer in CCDC26 were early events while the complex amplifications of the MYC coding sequence occurred later in tumor development.

\section{Complex-SV DMGs are associated with shorter survival}

We suspected that the fundamental differences in SV-generating processes across DMGs could associate with clinical phenotypes including survival. First, we confirmed the known association ${ }^{57,69}$ between the H3.1 ${ }^{\mathrm{K} 27 \mathrm{M}}$ mutation and longer overall survival (OS) compared to H3.3 ${ }^{\mathrm{K} 27 \mathrm{M}}$ (9.3 vs 16.1 months; $\mathrm{p}=0.0004$; Fig. 6D top), and the lack of association between TP53 mutation and OS within H3.3 ${ }^{\mathrm{K} 27 \mathrm{M}} \mathrm{pHGGs}(\mathrm{p}=0.72$; Fig. $6 \mathrm{D}$ bottom). To address SV signatures specifically, we also investigated the correlations between the numeric values of the combined Complex-SV signature and OS. Across all DMGs, this Complex-SV signature was significantly anti-correlated with OS (Fig. 8A; $p=0.001$ ).

We also found the combined Complex-SV signature to be significantly associated with shorter survival in a multivariate Cox regression analysis of DMGs that controlled for the known predictors of survival ${ }^{3,57}$ (Histone-SNV and age) and for TP53 status (Fig. 8B; $\mathrm{p}=0.038$ ). This analysis confirmed a significantly increased hazard ratio for $\mathrm{H} 3.3^{\mathrm{K} 27 \mathrm{M}}$ compared to both $\mathrm{H} 3.1^{\mathrm{K} 27 \mathrm{M}}$ and $\mathrm{H} 3^{\mathrm{WT}} \mathrm{DMGs}$, and a lack of significant associations between TP53 disruptions and OS in multi-variate analyses as previously described ${ }^{57}$. However, associations with age did not reach significance, probably due to our low representation of the under-three and over-ten age groups. While all patients with DMGs in our study died from their disease, the combined effects of these factors caused survival differences of several months. For example, children with DMGs with at least $20 \%$ Complex-SV activity survived a median of 9.6 months, about 3 months less than the 12.3 -month survival of children with less than $20 \%$ ComplexSV activity (Fig. 8C).

\section{Discussion}

Over the last decade, several landmark studies ${ }^{4-7}$ have revealed tremendous insights into the recurrent driver combinations of SNVs and SCNAs in $\mathrm{pHGG}^{3}$, including the sentinel discovery of the role of histone mutations as a founding alteration that shapes the genetic, epigenetic, and clinical features of these tumors ${ }^{8,70}$. However, the genome-wide landscape of SVs has not been described across a large cohort of pHGGs, particularly treatmentnaïve DMGs. We addressed this by leveraging novel analytical tools developed by our group to sensitively detect 
both individual and recurrent SVs ${ }^{22,36}$ and a large cohort of treatment-naïve DMGs made available by the DIPGBATS trial ${ }^{35}$, the first US multi-institutional trial to incorporate pontine biopsies at diagnosis. This analysis revealed novel structural variant signatures that distinguish pHGGs with predominantly simple vs complex SVs, and that these signatures, together with histone mutation status, determine the recurrent driver combinations and clinical outcomes. Among these are novel recurrent SVs, including a tandem duplication in $12 \%$ of all DMGs that colocalizes with a MYC enhancer within the IncRNA CCDC26.

Our analyses of structural variants in PHGG highlight a previously underrecognized role for MYC pathway activation in this tumor type. MYC is the most frequently amplified gene in all of cancer, with focal amplifications observed in $15 \%$ of tumors ${ }^{58,71}$. In contrast, similar MYC amplifications are only present in $5 \%$ of all pHGGs ${ }^{3}$. The discovery of recurrent amplification of the MYC enhancer in CCDC26 in pHGGs, without amplification of the MYC coding sequence itself, starts to address this discrepancy. The difference in how MYC is activated in pHGGs also extends to the location of $C C D C 26$ duplication. While tissue-specific amplifications of MYC enhancers have been previously reported in other cancers ${ }^{41}$, the CCDC26 duplication is in a novel location apparently driven by differences in enhancer landscapes across cell types. Altogether, when adding in high-level MYCN amplifications as well, we observe $14 \%$ of all pHGGs to harbor SVs predicted to activate MYC pathways. Given this high rate, the role of MYC activation in regulating cell-fate decisions and enhancing glioma formation in both histone-mutant and wild-type gliomas requires further study.

Although both Simple- and Complex-SV pHGGs activate MYC signaling pathways, they do so in strikingly different ways. While pHGGs with Simple-SV signatures amplify only the MYC enhancer, pHGGs with Complex-SV signatures contain high-level amplicons of both the MYC coding sequence and the enhancer regions - often extrachromosomal, as indicated by their circular topology. In this respect, MYC serves as an example for other oncogenes, including MYCN, PDGFRA, EGFR and MET. Extrachromosomal amplicons that contain both oncogenes and recurrent regulatory elements have also been observed in adult high-grade gliomas and other cancers ${ }^{13,21,72}$. However, their regulatory elements are different from pHGGs and appear to reflect the exquisite tissue specificity of these regulatory loci ${ }^{20}$.

Extrachromosomal amplicons (also known as double minutes, or DMs) have been shown to originate as byproducts of chromothripsis ${ }^{11}$. Our data suggest that, in $\mathrm{pHGG}$, they often contain multiple oncogenes from different chromosomes. Based on the proposed theory of their origin, an extrachromosomal amplicon with oncogenes from different chromosomes would therefore either require simultaneous chromothripsis of two chromosomes or need to develop sequentially by a less-clear mechanism. Our data also suggest that within a pHGG, the extrachromosomal amplicons often have multiple variants, which could correlate to different descendants of the initial DM as observed in recent mechanistic work ${ }^{11}$ and a prior long-read sequencing study of a single tumor from this study ${ }^{15}$. In cases where two oncogenes are integrated into a DM that is subsequently amplified, the number of copies of each oncogene should be identical. However, we find that pHGGs often exhibit different amplification levels of these oncogenes, suggesting sequential incorporation into the amplicon. The exact mechanism for this remains elusive and could range from sequential chromothripsis events ${ }^{11}$ to reversible DM integration in proximity to oncogenes as observed in neuroblastoma ${ }^{12}$, or possibly deletions within the DMs. It is tempting to speculate that the rapid evolution and optimization of $\mathrm{DMs}^{14,50}$ could contribute to the rapid, lethal growth of pHGGs and their poor response to any available therapies, similar to adult $\mathrm{GBM}^{73}$. Developing RTK inhibition is still a promising therapeutic goal in $\mathrm{pHGG}^{4,74,75}$, but our study highlights that understanding how these extrachromosomal amplicons evolve might inform how pHGGs become resistant. 
As the largest study of WGS data within a glial lineage, we expanded on the SV signatures that have been detected in other cohorts, allowing us to explore the contribution that glial lineages make to the repertoire of SV signatures. SV-signature analysis approaches were recently published for breast ${ }^{26}$, ovarian ${ }^{27,28}$ and other cancer types ${ }^{23}$. Intriguingly, we observe distinct SV patterns and associations with genetic variants compared to those other lineages. While tandem duplications have previously been associated with homologous recombination (HR) deficiency with alterations of BRCA1/2, TP53, CDK12 and CCNE1 across breast ${ }^{29,30}$, ovarian and hepatocellular cancers $^{31}$, this did not extend to pHGGs. The tumors in our cohort that were enriched with tandem duplications the Simple-SV tumors - were associated with none of these, and in fact tended to lack mutations in TP53 in favor of alterations of PPM1D and MDM4. However, the original associations were found in adult cancer types with markedly higher numbers of $\mathrm{SVs}^{23}$ and different genetic drivers than $\mathrm{pHGG}$. The contribution of lineage context and pHGG specific variants including the histone mutations to the formation of tandem duplications requires further study.

One association with histone mutations that we did observe was between $\mathrm{H} 3.3^{\mathrm{K} 27 \mathrm{M}}$, Complex-SV signatures, and loss of TP53. While the association between TP53 disruption and a higher SV burden has been described previously ${ }^{76,77}$, the reason for its association with $\mathrm{H} 3.3^{\mathrm{K} 27 \mathrm{M}}$ mutations over $\mathrm{H} 3.1^{\mathrm{K} 27 \mathrm{M}}$ is not understood. Similar to pioneering work in medulloblastoma showing that TP53 disruption precedes and might facilitate survival after chromothripsis $^{78}$, we also found TP53 disruption to be an early event in tumors with complex SVs. Notably, while almost all DMGs with Complex-SV signatures were TP53 disrupted, not all TP53-disrupted H3.3 ${ }^{\mathrm{K} 27 \mathrm{M}}$ DMGs showed Complex-SV signatures. Therefore, additional features might determine whether a PHGG acquires Complex-SV signatures. Additionally, hemispheric pHGGs with Complex-SV signatures were frequently TP53 ${ }^{\mathrm{WT}}$ but tended to harbor early loss of CDKN2A/B. This indicates that while TP53 loss and $\mathrm{H} 3.3^{\mathrm{K} 27 \mathrm{M}}$ are tightly correlated with Complex-SV signatures they are neither necessary nor sufficient, either alone or in combination, for the generation of the Complex-SV signatures in pHGG.

We found a reportable alteration of a known cancer gene in $98.3 \%$ (176/179) of patients, significantly expanding the share of patients with identified potential driver variants compared to prior studies that were based largely on exome sequencing. Many of the alterations that we observed were in non-coding regions of the genome, targeting regulatory elements such as enhancers. Moreover, use of whole-genome sequencing data allowed us to determine which patients had Complex- or Simple-SV signatures, which itself is associated with survival, even when controlling for histone and TP53 status. These findings indicate that both research and clinical sequencing of these tumors should encompass the whole genome.

We are in the process of transferring all novel sequencing data to dbGaP. Once this process is complete the data will be available at phs002380.v1.p1

Code Availability

Publicly available software was used as indicated in the methods. Custom code to connect and reformat the outputs of the publicly available software as well as code to generate the figures is available upon request. 


\section{Acknowledgements}

530 We thank and acknowledge the children and their families who made this research possible through their participation in pHGG clinical trials and tissue banking protocols. We also thank the many clinicians and 532 researchers who led the DIPG BATS trial. We would also like to thank the members of the Ligon, Beroukhim and Bandopadhayay Laboratories, in addition to Ruolin Liu, Justin Rhoades, Matthew Leventhal, Justin Hwang, 534 Jonathan So, David Mayhew, Jonathan Rennhack, Mehdi Touat, Andrew Cherniack, Beatrice Rondelli and Sophie Polo for their support and helpful discussions.

536 The authors would like to thank and acknowledge the following funding sources:

The German Research Foundation (DFG) (research fellowship supporting FD), Fund for Innovative Cancer 538 Informatics (RB), the Gray Matters Brain Cancer Foundation (RB), The Bridge Project of MIT and DanaFarber/Harvard Cancer Center (RB, KLL), The Sontag Foundation (RB), The V Foundation for Cancer Research (PB), 540 Michael Mosier Defeat DIPG Foundation (PB), The ChadTough Foundation (PB), The St. Baldrick's Foundation (RB, $\mathrm{PB}, \mathrm{JWT}$ ), Prayers from Maria Foundation (PB, MWK), Pediatric Brain Tumor Foundation ( $\mathrm{PB}, \mathrm{RB})$, Friends of DFCl

542 ( $P B, R B$ ), The Giving for Gabi Fund (PB), McKennaClaire Foundation (PB, MWK), We Love You Connie Foundation (PB, RB, MWK, NJ), Imagine for Margo and Lemos Family (JG), The Pussycat Foundation Helen Gurley Brown 544 Presidential Initiative (JWT, PB), Canadian Institutes of Health Research (NJ, CLK), Zach Carson Fund (MWK, RB, PB), Ellie Kavalieros DIPG Fund (MWK, RB, PB), Ryan Harvey Fund (MWK, RB, PB), Mikey Czech DIPG Foundation 546 (MWK, RB, PB), Caroline Cronk Fund (MWK, RB, PB), Markoff Art in Giving Foundation (MWK, RB, PB), Brock Fleming Fund (MWK, RB, PB), Stop and Shop Pediatric Brain Tumor Program (MWK, RB, PB, KDW, SNC), the DIPG 548 Collaborative (MWK, RB, PB), The Cure Starts Now (RB)

FD, MWK, KLL, PB, RB conceived the project. FD, OS, NG, TZ, JW, JT, DH, AC, AH, KK, MBJ, KSK, CS, DW, PK, HM, $552 \mathrm{PH}, \mathrm{RR}, \mathrm{RG}, \mathrm{DD}, \mathrm{JS}, \mathrm{MS}, \mathrm{KW}, \mathrm{SC}, \mathrm{JG}, \mathrm{CK}, \mathrm{LG}, \mathrm{NJ}, \mathrm{DJ}, \mathrm{MWK}, \mathrm{KLL}, \mathrm{RB}, \mathrm{PB}$ acquired samples, generated and analyzed the data. FD, RB, PB wrote the manuscript, all authors edited the manuscript. KLL, PB and RB supervised the overall 554 study.

\section{Conflict of Interest}

RB and PB receive grant funding from the Novartis Institute of Biomedical Research for an unrelated project and RB receives grant funding from Merck. P.B consults for QED Therapeutics, R.B. consults for and owns equity in Scorpion Therapeutics, reports consulting or advisory role for Novartis, Merck (I), Gilead Sciences (I), ViiV Healthcare (I); research funding from Novartis; patents, royalties, other intellectual property-Prognostic Marker for Endometrial Carcinoma (US patent application 13/911456, filed June 6, 2013), SF3B1 Suppression as a Therapy for Tumors Harboring SF3B1 Copy Loss (international application No. WO/2017/177191, PCT/US2017/026693, filed July 4, 2017), Compositions and Methods for Screening Pediatric Gliomas and Methods of Treatment Thereof (international application No. WO/2017/132574, PCT/US2017/015448, filed 1/27/2017). KLL reports grants and personal fees from BMS, grants from Amgen, personal fees and other from Travera LLC, personal fees from Rarecyte, grants from Tragara, grants from Lilly, grants from Deciphera, grants from X4, grants from Charles River Labs, grants from Specicare, outside the submitted work; has a patent US20160032359A1 pending. MWK is now an employee of Bristol-Myers Squibb. LCG is the Co-Founder of Thromboprotea Inc. All the remaining authors declare no competing interests. 


\section{Methods}

570

\section{Sample acquisition}

572 This study includes published ${ }^{4-7}$ data available under EGAS00001000575, EGAS00001001139, EGAS00001000572 and EGAS00001000192. Novel data was generated from samples obtained from the DIPG-BATs clinical trial, the Dana-Farber Tissue Bank or collaborating institutions, under protocols approved by the institutional review board of the Dana-Farber/Harvard Cancer Center with informed consent. DNA and RNA were extracted from single DMG cores, pHGG biopsies, and autopsy samples using Qiagen AllPrep DNA/RNA extraction kits. Previously published pHGG whole genome sequencing (WGS) data $^{4-7}$ and, if present, paired RNA-seq were acquired from public repositories.

Whole-genome sequencing

Library preparation for paired end whole genome sequencing (WGS) was performed as previously described ${ }^{17}$. In brief, genomic DNA was fragmented and prepped for sequencing (to 60X depth for tumors and 30X depth for normal samples) on an Illumina HiSeq 2000 instrument. Reads from both novel and published data were aligned to the reference genome hg19/ GRCh37 with BWA ${ }^{79}$, duplicate-marked, and indexed using SAMtools and Picard. Base quality score was bias adjusted for flowcell, lane, dinucleotide context, and machine cycle and recalibrated, and local realignment around insertions or deletions (indels) was achieved using the Genome Analysis Toolkit. All paired samples underwent quality control testing to ensure accuracy of tumor-normal pairs.

\section{SNV and SCNA analyses}

590 SNVs were detected using Mutect $2^{80}$ and filtered for common sequencing artifacts, gnomad SNPs, and SNVs present in a panel of whole genome-sequenced normal samples from blood from this cohort $(n=175)$ and TCGA $(n=5)$. Significance of recurrent SNVs in non-hypermutant samples (SNV counts $<100,000 /$ sample) was determined with MutSigCV ${ }^{81}$. SCNAs were called using the GATK4 somatic CNV pipeline with normalization against a panel of blood normals from 184 samples (174 from this cohort, 10 from TCGA) based on the Tangent method ${ }^{82}$. Purity and ploidy were determined using ABSOLUTE ${ }^{83}$. All somatic copy number alteration (SCNA) calls were purity- and ploidy-adjusted as previously described ${ }^{84}$. Significantly recurrent SCNAs were identified using GISTIC $2.0^{85}$ with the following parameters: amp_thresh $=0.5$; del_thresh $=0.7$; arm_peel=0; broad_length_cutoff $=0.5$; cap=3.6; conf_level=0.99; max_sample_segs=3500; and qv_thresh=0.1. Analyses were performed using the https://firecloud.terra.bio platform.

600

\section{Structural variant detection and significance analysis}

SVABA ${ }^{36}$ was used to call SV in paired tumor normal mode with default parameters. In addition to filtering germline variants against the paired normal, telomers and centromeres were blacklisted. The significance of recurrence analysis was performed separately for breakpoints (1D) and juxtapositions (2D) analogous to the previously published approach for pan-cancer ${ }^{22}$ and adapted to the pHGG context.

606 For the significance analysis of recurrent breakpoints (1D) as previously described ${ }^{22}$, the genome was binned into $50 \mathrm{kbp}$ bins with $500 \mathrm{bp}$ overlap. The bins were annotated by naming them after the TAD they were located in, with 608 the name of the TAD derived from the Cosmic Cancer Gene Census ${ }^{53}$ gene in the TAD. Germinal zone TAD boundaries from GSE $77565^{86}$ were used as the closest available normal neural progenitor. Eligible territory ${ }^{87}$ was

610 defined by masking low-complexity genomic loci based on https://github.com/lh3/sgdpfermi/releases/download/v1/um35-hs37d5.bed.gz. Only one SV per sample per bin was counted. Fishhook ${ }^{88}$ was

612 used to calculate a background model for the likelihood of a breakpoint in each bin, based on six covariates: replication timing (from http://mskilab.com/fishHook/hg19/RT_NHEK_Keratinocytes_Int92817591_hg19.rds), GC 
content (from http://hgdownload.cse.ucsc.edu/goldenPath/hg19/gc5Base/hg19.gc5Base.txt.gz), presence of SINE elements (http://www.repeatmasker.org), fragility

616 https://data.broadinstitute.org/pcawg6sv/1D_covariates/fragile_genes_smith.hg19fp.txt (adapted from ${ }^{89}$, mappability (from http://hgdownload.cse.ucsc.edu/goldenPath/hg19/encodeDCC/wgEncodeMapability/), and

618 heterochromatin status. ChromHMM for the caudate nucleus was chosen for the heterochromatin track based epigenetic similarity to pHGG (Ext. Data Fig. 2A). These data were downloaded from 620 https://egg2.wustl.edu/roadmap/data/byFileType/chromhmmSegmentations/ChmmModels/coreMarks/jointM odel/final/ E068_15_coreMarks_mnemonics.bed.gz and subset to ['8_ZNF/Rpts', '9_Het', '15_Quies',

622 '14_ReprPCWk'] to define heterochromatin. All these covariates have been repeatedly shown to be predictive of the probability of a SV occurring in the bin $^{22,23,88,90}$ and $p$-values for the influence of each individual covariate on 624 the model ranged from $2 \times 10^{-16}$ to 0.21 . $p$-values reflecting the significance of breakpoint enrichment were then calculated and corrected for multiple hypothesis testing using the Benjamini-Hochberg procedure ${ }^{91}$. If several 626 bins within one TAD were significant, they were considered as one significant locus and linked to any COSMIC Cancer Gene ${ }^{53}$ gene in that TAD.

Recurrent juxtapositions (the 2D analysis; Ext. Data Fig. 3A) were detected using a background model determined from 2658 cancers across several dozen cancer types ${ }^{22}$ and the same binning scheme (5583 bins with a median span of $467 \mathrm{kbp}$ and interquartile range of $347 \mathrm{kbp}$ ). Only one SV from each sample was allowed to contribute to connections between any two bins (a "tile"). Only SVs with at least four supporting reads and a span of $>1 \mathrm{kbp}$ were included in this analysis. p-values reflecting the significance of enrichment of SVs within each tile were corrected for multiple hypothesis testing using the Benjamini-Hochberg procedure ${ }^{91}$. Only significantly recurrent juxtapositions which did not occur at the same nucleotide position, had a mean SvABA-assigned quality score of larger than 20, included at least one SV detected with post-assembly (ASDIS or ASSMB) evidence, occurred in more than two samples, and had a q-value smaller than 0.05 were considered for further analysis.

\section{SV signature analysis}

640 SV signature analysis followed previously published approaches ${ }^{23,26}$, with slight modifications for overall lower SV counts in pediatric tumors. In brief, SVs were stratified according to the span between the two breakpoints (0$30 \mathrm{kbp}, 0.03-1 \mathrm{Mbp},>1 \mathrm{Mbp}$, interchromosomal); read orientation (deletion, duplication, inversion and interchromosomal); and whether they were clustered or simple based on the comparison between local SV density and the overall SV density in the sample, as determined by clusterSV ${ }^{23}$. This resulted in a count matrix of 20 SV feature combinations $x 179$ pHGGs, which was analyzed with Bayesian NMF using SignatureAnalyzer ${ }^{24,25}$ code to determine the optimal, most informative number of signatures and signature activities in each sample.

\section{RNAseq analysis}

RNAseq data were available for 112 of the 179 tumors ( 57 sequenced de novo and 55 previously published ${ }^{4,6}$ ). For de novo samples, cDNA libraries were prepped as previously described ${ }^{17}$ using the Tru-Seq Strand Specific LargeInsert kit, and sequenced to a depth of 50 million paired ends using Illumina Hi-Seq machines. Reads from both de novo and published data were aligned to the hg19 reference genome using STAR ${ }^{92}$ and quantified with RNA$\mathrm{SeQC}^{93}$ following the GTEX analysis pipeline ${ }^{94}$. Counts were normalized using the VST transform as implemented in DESeq $2^{95}$ and batch-corrected with COMBAT ${ }^{96}$ as implemented in sva ${ }^{97}$.

Previously published H3K27ac ChIP-seq sequencing data from primary DMGs were downloaded from GSE128745 ${ }^{42}$. Peaks were called using MACS2 ${ }^{98}$ callpeak with -B -SPMR to save the fragment pileup per million 
660

662

664

666

668

670

672

674

676 Data processing

Additional Hi-C files for NPCs (neural progenitor cells) were downloaded 678 which were transformed into bigwig files with rtracklayer and visualized together with copy number and SV calls in gtrack. Additional bigwig files for adult GBM H3K27ac, pHGG ATAC seq and non-cancerous or non-brain tissues were downloaded from GSE54792 ${ }^{43}$, GSE126319 44 and the Encode project ${ }^{45,46}$ respectively.

Hi-C

Library generation and sequencing

In-situ Hi-C libraries were generated from 5 million cultured H3.3 ${ }^{\mathrm{K} 27 \mathrm{M}}$ glioblastoma cell lines HSJ-019 and HSJ-031 as well as from a $\mathrm{H} 3.3^{\mathrm{K} 27 \mathrm{M}}$ primary tumor HSJ-031 following published protocols ${ }^{99}$ with minor modifications. Briefly, in situ Hi-C consists of 7 steps: (1) crosslinking cells with formaldehyde, (2) digesting the DNA using a 4cutter restriction enzyme (e.g., Dpnll) within intact permeabilized nuclei, (3) filling in, biotinylating the resulting 5 '-overhangs and ligating the blunt ends, (4) shearing the DNA, (5) pulling down the biotinylated ligation junctions with streptavidin beads, (6) library amplification and (7) analyzing these fragments using paired end sequencing. Quality control (QC) for efficient sonication was performed through the combination agarose DNA gel electrophoresis and for appropriate size selection using the Agilent Bioanalyzer on final amplified libraries, followed by low-pass sequencing on the Illumina HiSeq 2500 ( 30M reads/sample) to assess quality of the libraries using percent of reads passing filter, percent of chimeric reads, and percent of forward-reverse pairs.

from www.synapse.org/\#!Synapse:syn12979101 (registration required; Data Download - Study “iPSC-HiC") ${ }^{100}$. Analysis of $\mathrm{Hi}-\mathrm{C}$ generated and downloaded fastq files was performed using Juicer and the associated Juicer Tools $^{101}$. Contact maps were generated using Juicer with the following parameters: -s Dpnll -g hg19. Map resolution was determined by using Juicer's "calculate_map_resolution.sh" script. Hi-C contact maps and associated annotations were visualized using Juicebox ${ }^{101}$. The HIFI algorithm ${ }^{102}$ was used to process $5-k b$ resolution $\mathrm{Hi}-\mathrm{C}$ data to obtain higher accuracy estimates of interaction frequencies, using the following parameters: bandSize $=1000$, outputNormalized, boundaryKS=1000.

\section{Luciferase reporter}

Cell lines.

The tumor tissue derived pHGG cell line DIPG13 ${ }^{103}$ was a gift from the Michelle Monje lab. Cell lines were grown in ultra-low attachment flasks in a 1:1 ratio of Neurobasal A (Gibco) and DMEM/F-12 (Gibco) and one percent of each HEPES Buffer Solution 1M, Sodium Pyruvate Solution 100nM, MEM Non-Essential Amino Acids Solution $10 \mathrm{mM}$, Glutamax-I Supplement, and Penicillin/Streptomycin solution. The culture medium was supplemented with epidermal and fibroblast growth factor (H-EGF \& H-FGF; StemCell Tech, Inc.) at $20 \mathrm{ng} / \mathrm{mL}$, platelet derived growth factors (H-PDGF-AA \& H-PDGF-BB StemCell Tech, Inc.) at $10 \mathrm{ng} / \mathrm{mL}$, Heparin Solution (0.2\%; StemCell Tech, Inc.) at $2 \mathrm{ug} / \mathrm{mL}$, and 50X B-27 Minus Vitamin A (Invitrogen). Cells were regularly passaged every 2-4 days to avoid confluence and neurospheres were dissociated into single cells at the time of passage using Accutase (StemCell Tech, Inc.) to prevent overly tight neurospheres.

\section{Cell line authenticity and mycoplasma surveillance}

SNP-based fingerprinting assays were performed at regular intervals to ensure authenticity of cell-lines. All cell lines were routinely monitored for mycoplasma infection using the MycoAlert Mycoplasma Detection Kit (Lonza), following the manufacturer's protocol.

reads track. The resulting bdg-files were used to calculate fold enrichment and q-value tracks with macs 2 bdgcmp 
A lentiviral firefly luciferase reporter system was constructed from pGL4.26 (Promega) and the pLKO.1 backbone via Gibson Assembly. The pLKO.1 backbone was digested with FastDigest ${ }^{\circledR}$ KflI and EcoRI. The minimal promoter firefly reporter cassette was PCR amplified from pGL4.26 using the lucminP primer set (Supp. Table 6) using NEB Q5 polymerase. These two fragments were assembled into the lentiviral firefly luciferase reporter using the NEBuilder HiFi DNA Assembly Cloning Kit according to manufacturer's instructions. The DNA sequence in the H3K27ac peak in the consensus CCDC26-SV amplicon was split into two fragments (E1/E2) and PCR amplified from DIPG13 genomic DNA with the primers listed in Supp. Table 6 using NEB Q5 polymerase. The resulting E1 and E2 fragments were cloned into the vector, upstream of the minimal promoter in the lentiviral firefly luciferase reporter system using KPN1 and NHE1 restriction sites. The lentiviral constitutively active pLX313-Renilla construct was obtained from addgene (Plasmid \# 118016) to serve as intrinsic control. All reporter experiments were conducted in the DIPG13 cell line constitutively expressing Renilla luciferase from this pLX313-Renilla construct.

Viral Production

HEK-293T cells were cultured in T75 tissue culture treated flasks in DMEM (Gibco) supplemented with 10\% FBS (Gemini Bio). Lipofectamine-3000 (Invitrogen) was used to transfect with plasmid of interest in addition to packaging plasmids VSV-G and psPAX2, according to manufacturer's protocol. Media was replaced with DMEM supplemented with 20\% FBS six hours after transfection. Media was harvested 24-48 hours post-transfection and virus concentrated (20x) using Lenti-X Concentrator (Takara Bio) per manufacturer's protocol.

Lentiviral infection

Cells were dissociated and plated in a 12-well tissue culture plate at a density of 1.5 million cells $/ \mathrm{mL}$. Concentrated

virus was added to the media and the cells were centrifuged for 120 minutes at 2000 RPM and $30 \mathrm{C}$. Following centrifugation, cells were harvested and placed into T-75 ultra-low attachment flasks. Selection $(1 \mu \mathrm{g} / \mathrm{ml}$ puromycin for the firefly reporter, $300 \mu \mathrm{g} / \mathrm{ml}$ for pLX313-Renilla) was added the following day to achieve survival of $40-80 \%$ in the infected conditions.

\section{Luciferase Reporter Readout}

730 The Dual-Glo Luciferase Assay System (Promega) was used following manufacturers protocols for all measurements. In brief, the firefly reading for each reporter condition was first normalized to the constitutive

732 Renilla luciferase signal. The final reporter values are gives as fold change of the E1 or E2 normalized firefly luciferase signal compared to the empty lentiviral luciferase reporter four days post spinfection (two days post 734 puro selection).

Visualization and reconstruction of complex MYCN and RTK amplicons $\mathrm{JaBbA}{ }^{90}$ was used to generate cancer genome graphs using SVABA SV, GATK CNV and absolute purity and ploidy 738 as inputs. The cancer genome graphs combined with ChIP-seq tracks were visualized in gGnome (https://github.com/mskilab/gGnome) / gTrack (https://github.com/mskilab/gTrack), which were also used to 740 calculate distances between loci in the cancer genome. To infer the structure of possible extrachromosomal amplicons the genome graphs were subsetted to only segments with $\mathrm{CN}>20$ and all circular paths in the genome graphs were reconstructed with the gGnome walks() function.

744 To determine which genomic loci were recurrently incorporated into the amplicons, the distribution of amplicons around the oncogene was evaluated using methods analogous to previously published approaches ${ }^{20,21}$. First, the TADs adjacent to the amplified oncogene were divided into $10 \mathrm{kbp}$ windows. The average copy number per $10 \mathrm{kbp}$ 
window was calculated in all tumors with an amplicon of $\mathrm{CN}>5$ anywhere in the TAD of the oncogene (using the germinal zone TAD boundaries from GSE $77565^{86}$ ). Likewise, among tumors with an amplification of $\mathrm{CN}>5$ anywhere within the TAD of the oncogene, the fraction of tumors with an amplification in each $10 \mathrm{kpb}$ window was determined. If the fitness advantage provided by the amplicons resulted only from their inclusion of the oncogene, one would expect a symmetric normal distribution with the peak at the location of the coding sequence of the oncogene for both mean amplification level and fraction of tumors with amplifications in the 10kbp windows around the oncogene. The location of likely enhancer elements, necessary to drive expression of the amplified oncogene, was inferred from the direction of the skew of the observed distribution compared to the expected symmetric normal distribution.

\section{SNV signature analysis}

758 De novo SNV signature extraction was performed using Bayesian NMF in SignatureAnalyzer, as previously described ${ }^{24}$. The resulting SNV signatures were compared to the COSMICV3 SBS signatures using cosine similarity to annotate known etiologies and signature names.

Signature integration and definition of signature clusters with similar variant generating processes

To better understand the information contained in each of the five SV and 14 SNV signatures, consensus clustering was applied to the tumor $x$ signature proportion matrix, comprising the 19 values representing the proportion of each of the SV and SNV signatures out of all SV and SNV signatures in each tumor. The proportions for each signature were median-centered across all tumors before consensus clustering with the ConsensusClusterPlus $R$ package ${ }^{104}$ using the parameters: reps $=1000$, pltem $=0.9$, pFeature $=0.9$, clusterAlg="hc",distance="spearman". The resulting three most stable and informative clusters were named "Complex-SV", "Intermediate" and "Simple-SV", after the signature with the highest enrichment in the cluster.

\section{Comut plots and variant combination matrix}

An annotated list of all SNVs, SCNAs and SVs was created for all tumors in this cohort. SNVs were annotated using Oncotator ${ }^{105}$. SVs were annotated and linked to a gene based on if the SV breakpoints where located in exons of the gene (named 'coding SV'), intronic ('intron SV') or in the TAD of the gene ('flank SV'). Absolute purity- and ploidy-adjusted copy number (CN) was determined for every gene using the width-weighted mean $\mathrm{CN}$ from all segments overlapping the gene.

To create the variant combination matrix, this list of all variants in all genes from all tumors was subsetted to only contain variants in genes from the Cancer Gene Census ${ }^{53}$, in addition to genes which showed significantly recurrent variants in this cohort. For SNVs, the variant classification was simplified to truncating_snvs = ("Nonsense_Mutation", "Frame_Shift_Del", "Frame_Shift_Ins", "Splice_Site", 'Start_Codon_SNP', 'START_CODON_SNP', 'Translation_Start_Site') and missense.snvs = ("Missense_Mutation", "In_Frame_Del", "Stop_Codon_Del", 'DE_NOVO_START_IN_FRAME', 'DE_NOVO_START_OUT_FRAME', 'Nonstop_Mutation',

784 'In_Frame_Ins', 'START_CODON_INS'). SCNAs of genes with a ploidy- and purity-adjusted copy number of $<0.2$ were annotated as 'homdel', CN > 5.4 as amp, CN > 10 as ExChr_amp and amplifications covering only parts of a gene with a $\mathrm{CN}>3.1$ as 'part.amp' based on the $\mathrm{CN}$ histogram across all tumors defining recurrent $\mathrm{CN}$ states. Using the $\mathrm{R}$ data.table dcast function on the gene variants and samples, binary matrix of samples $\mathrm{x}$ variants was created.

788 A genetic variant had to recur in at least three samples (excluding the hypermutant samples for SNVs) to be kept in the variant $x$ sample matrix. For SV in the TAD of a gene ('flank SV') this threshold was increased to at least ten because of their higher occurrence probability based on the large size of TADs compared to genes. The presence or absence of the GISTIC peaks in each sample was used to incorporate the SCNAs of lower amplitude. 
This procedure resulted in the binary variant combination matrix of 176 unique tumors by 353 variants (Three 794 tumors showed no recurrent variants) which was used as the basis for all enrichment and variant clustering analyses. Additionally, 16 significantly recurrent arm level SCNAs were included for enrichment analyses but excluded from the variant clustering as they are highly correlated with the GISTIC peaks.

798 The cbioportal ${ }^{106,107}$ oncoprinter was used to visualize the variants in the tumor samples. The order of the columns representing the tumor samples within each subgroup was determined by hierarchical clustering (HC). $\mathrm{HC}$ with one minus spearman rank correlation metric was applied on the signature activities in each tumor for Fig. 4B and hc with one minus cosine similarity metric on the respective subsets of variant combination matrix for Fig. $5 \mathrm{~A}$ and Fig. $6 \mathrm{~A}$ with average linkage in all cases. Genes of interest were manually selected based on the variants with the highest enrichment in the subgroups.

\section{Variant timing analysis}

806 Previously published approaches ${ }^{61,108}$ were leveraged to evaluate if a potential driver variant occurs early or late in tumor development. The palimpsest ${ }^{109} \mathrm{R}$ package was applied to determine single patient timing of SNVs, as 808 previously described ${ }^{108}$. In brief, SNVs were classified into clonal vs. subclonal based on their cancer cell fraction (CCF, calculated from the variant allele fraction adjusted by local copy number and purity/ploidy for the respective sample). SNVs overlapping with SCNA could further be timed into early or late depending on if they happened before or after the SCNA ${ }^{61}$. MutationTime ${ }^{61}$ was used to determine the timing of SCNAs in individual patients, including clone clusters as input. Mobster ${ }^{110}$ was used to define the clone clusters based on the distribution of the absolute ${ }^{83}$ CCFs. The resulting molecular time for the SCNA segments was assigned to the GISTIC peaks present in the respective sample with a width-weighted mean and categorized based on the timing quarters. For each subgroup, in addition to the timed GISTIC peaks, single patient timed SNVs in consensus cancer genes were tallied into winning tables reflecting the frequency of this variant being an early event using published code ${ }^{108}$. In the resulting winning tables each variant competes against all other variants in the table. For each comparison the earlier variant wins. The BradleyTerryScalable R package was applied to estimate the winning probability across the subgroup for each variant, which in this setting is a measure for the probability of this variant being an early event in the tested subgroup. The Bayesian maximum a posteriori probability (MAP) estimate was used to fit the model as previously described ${ }^{108}$. To control for outlier samples the analysis was performed on 100 random samples of $70 \%$ of each subgroup. The resulting distributions for the strength parameters (on log scale) were plotted for variants recurrent at least three times in the tested subgroup.

\section{Survival analysis}

826 Univariate correlations for differences in survival were analyzed using the Kaplan-Maier method and significance was determined with a log-rank test. Spearman rank correlation tests were used to determine correlations between overall survival and the complex-SV signature activity. This was possible because all children with DMGs died within the observed period, resulting in an absence of censored data. Variables included in the multivariate analysis (Cox-model) were Histone-SNV, age, TP53 status combined Complex-SV signature activity.

\section{Statistical analysis}

All statistical analyses were performed in R 3.6.3. Unless otherwise indicated, statistical comparisons were

performed using Fisher's exact tests or Wilcoxon tests, as appropriate. $p$-values $<0.05$ were considered significant. Multiple testing was accounted for by using false discovery rate ${ }^{111} q$-values unless otherwise indicated. In all box 
836 plots the boxes represent the range between the $25^{\text {th }}$ and $75^{\text {th }}$ percentiles and the central line indicates the median.

838 
Figures and figure legends:
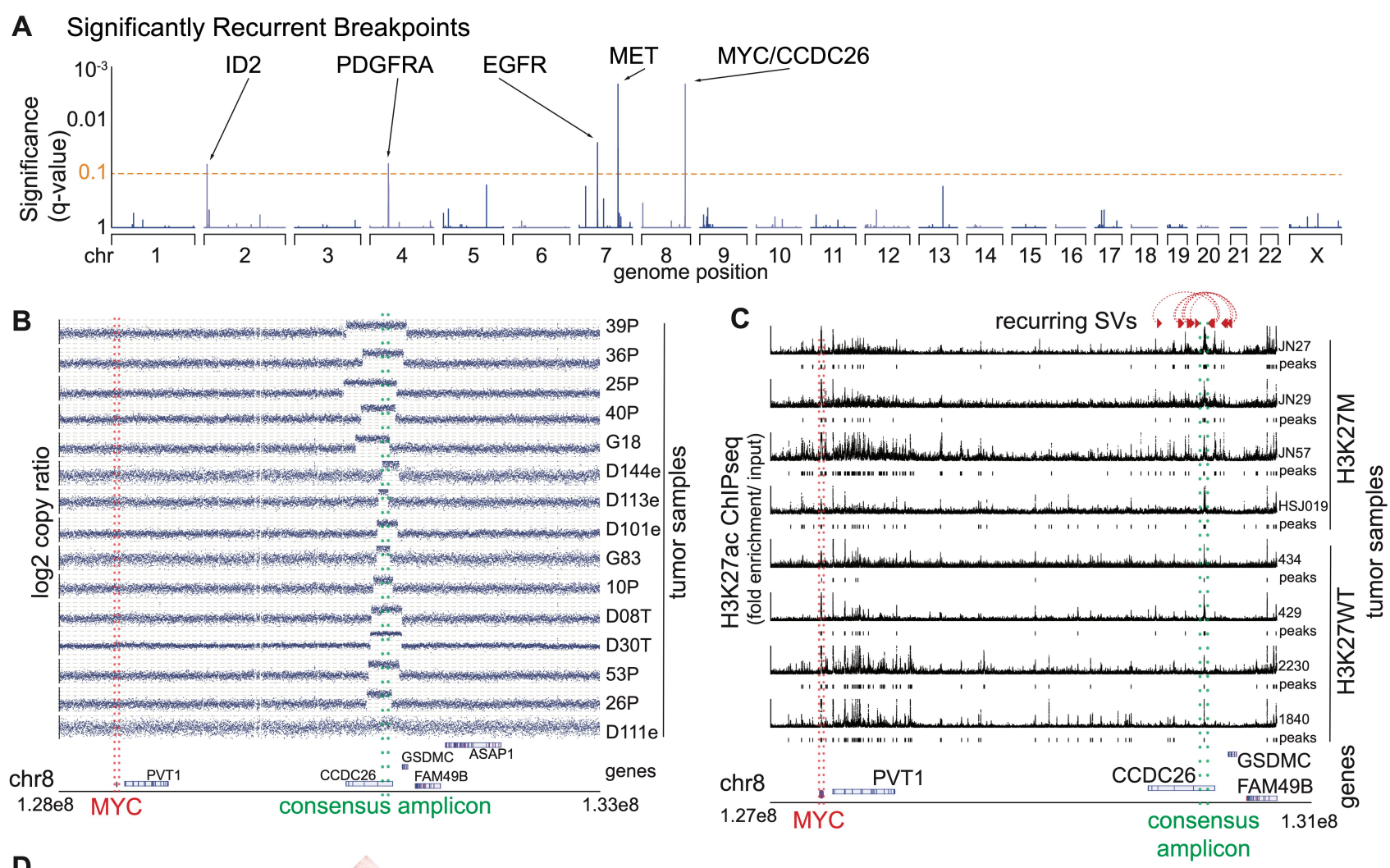

D
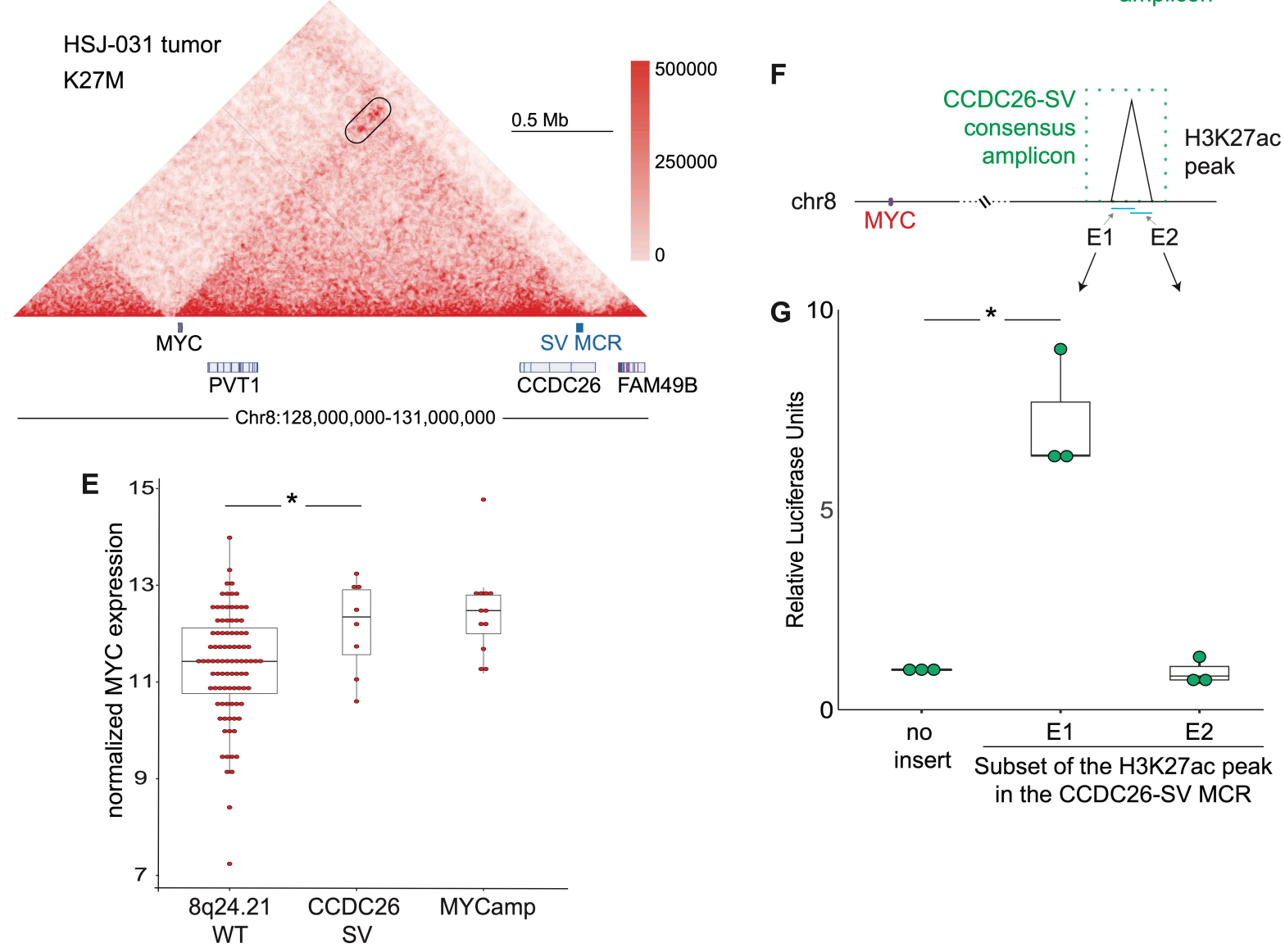

Figure 1 
Significantly recurrent breakpoints within CCDC26. (A) Significance (multiple hypothesis corrected q-values, 844 vertical axis) of recurrent breakpoints (genomic positions on the horizontal axis) across the 179 pHGG genomes. (B) Copy-number profiles across the MYC TAD for the 15 tumors with the recurrent CCDC26 SV. (C) H3K27ac ChIP846 seq tracks within the TAD containing MYC (red lines) and CCDC26, for four H3 ${ }^{\mathrm{K} 27 \mathrm{M}}$ and four H3K27 ${ }^{\mathrm{WT}}$ pHGGs tumors. Significantly enriched peaks (q-value < 0.01) are indicated below each H3K27ac ChIP-seq track. The CCDC26 848 amplicon boundaries for individual samples are indicated by the paired red arrows at the top. The consensus amplicon is indicated by the green dotted lines, and centers on an H3K27ac peak. (D) Hi-C heatmap across the 850 MYC-CCDC26 locus from a midline glioma. Increasing interaction frequencies are indicated by brighter shades of red. The black oval outlines the frequent connections between MYC and the H3K27ac-enriched region within 852 CCDC26. The minimal common region of amplification (SV MCR) is indicated at the bottom. (E) Normalized MYC expression in DMG samples with wild-type copy-number profiles at 8q24.21 ( $n=92)$, CCDC26-SVs $(n=8)$ or 854 amplifications of the MYC coding sequence $(n=12)$. *denotes a $p<0.05$ as determined by Wilcoxon rank sum test. (F) Schematic illustrating the luciferase reporter used to validate the enhancer in CCDC26, showing the positions 856 of the E1 and E2 sequences with respect to the enhancer within CCDC26. (G) Normalized luciferase activity in DIPG13 cells following transduction of the E1 and E2 enhancer reporters or empty vector controls. Values 858 represent the average of four technical replicates in each of three independent experiments. * denotes $p=0.019$, T-test (unpaired, two-sided). 
A

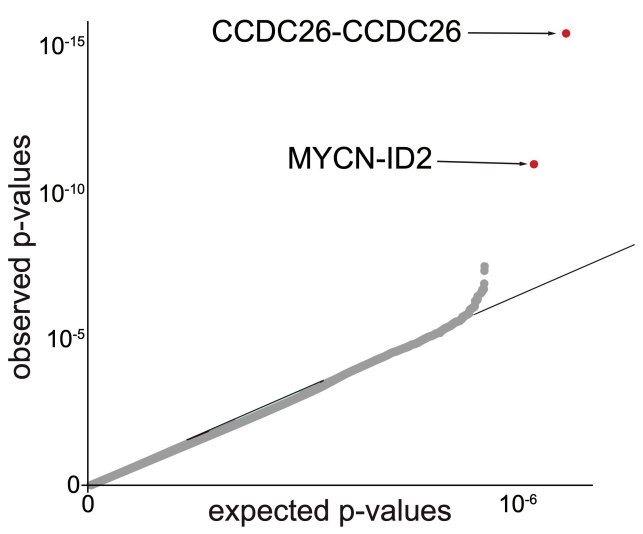

C

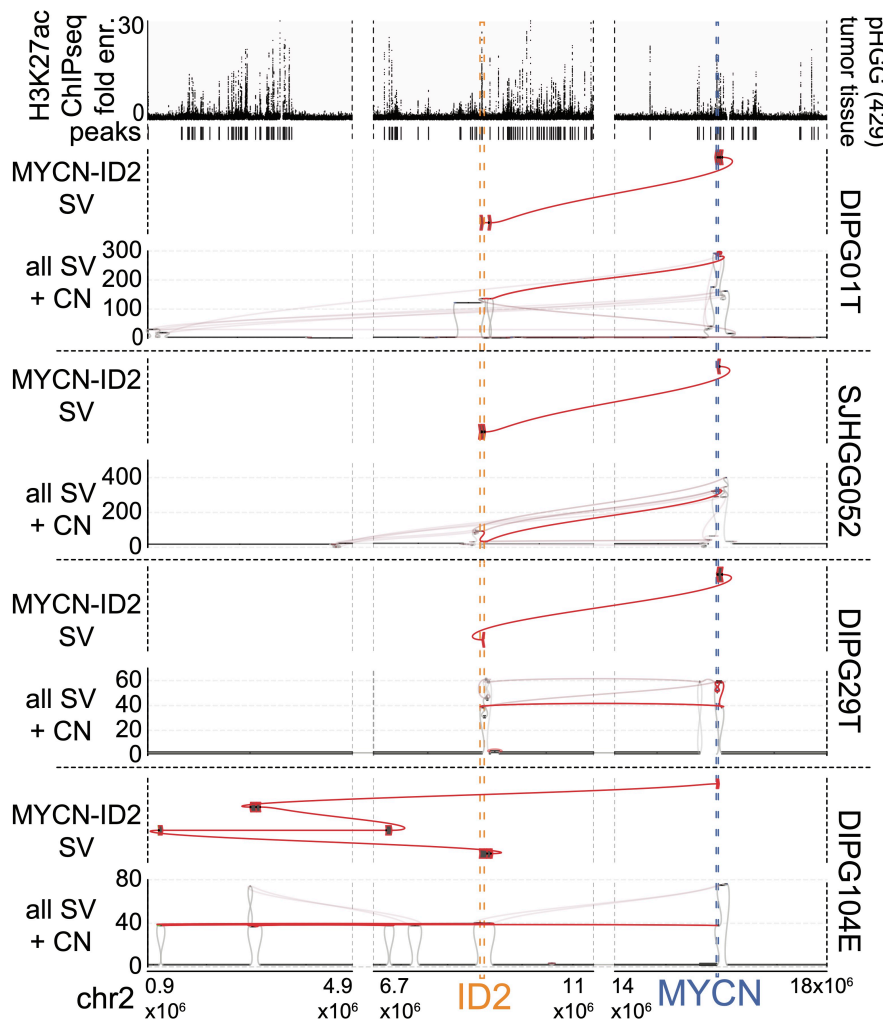

F

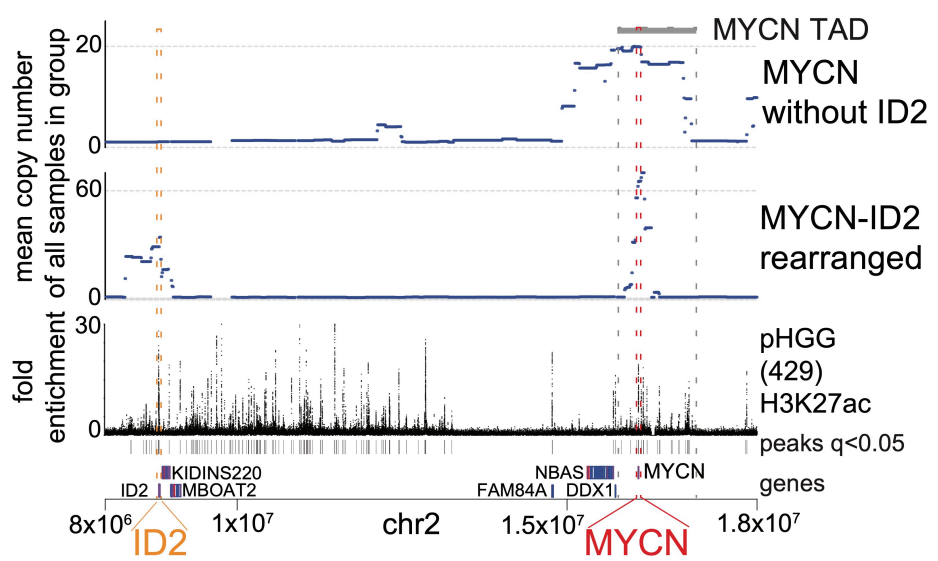

B

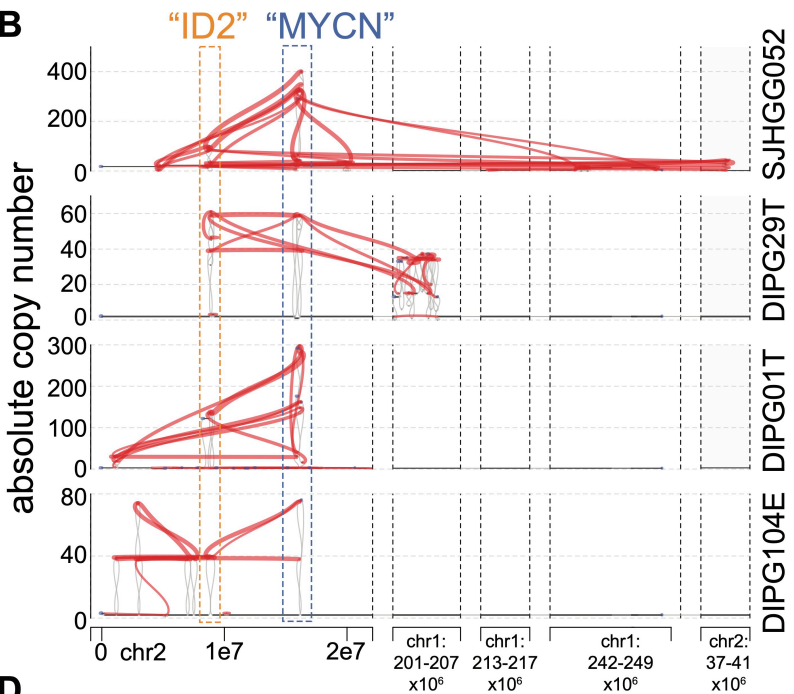

D

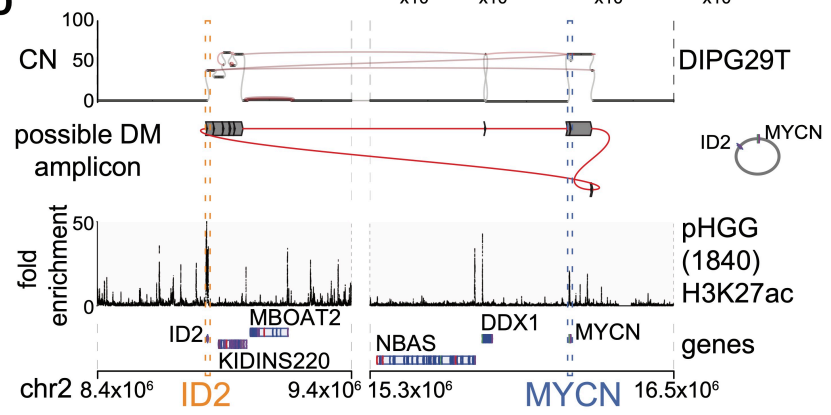

E
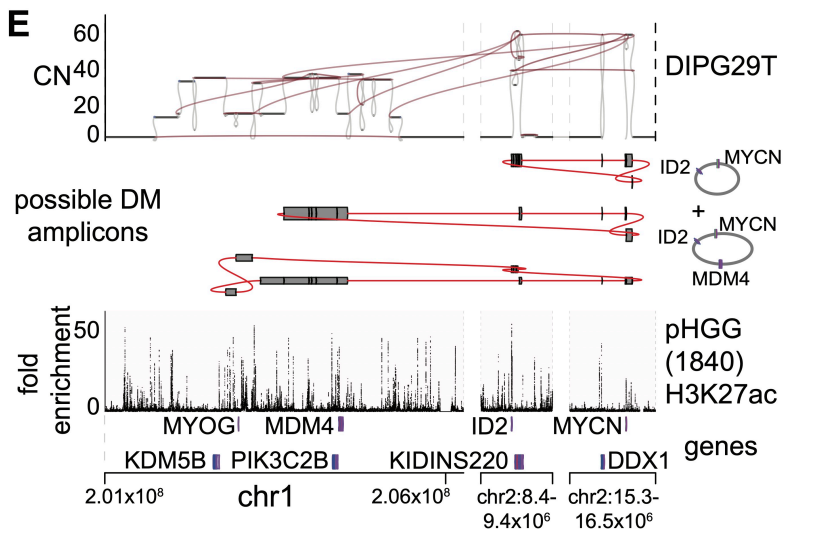

G

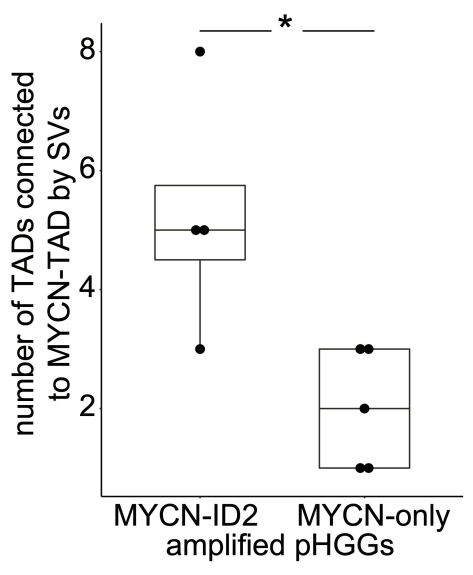




\section{Figure 2}

864 Significantly recurrent juxtapositions: MYCN-ID2. (A) Quantile-Quantile plot indicating the significance of juxtapositions between pairs of genomic loci. SVs that reached statistical significance are depicted in red. (B) SVs 866 (red lines) involving MYCN and ID2 in samples with MYCN-ID2 rearrangements, and the number of copies at each connected locus (vertical axis). The dashed boxes indicate loci encompassing ID2 and MYCN. (C) SV maps as in 868 panel B, with SVs juxtaposing ID2 and MYCN highlighted in red. The top track indicates H3K27ac marks in a pHGG without a known MYCN-ID2 rearrangement, showing a strong enhancer within ID2. In each case, the MYCN-ID2 870 juxtaposition reduces the somatic distance MYCN and ID2 (each indicated by a dashed line) from $7 \mathrm{Mbp}$ to less than 700kbp. (D-E) Examples of possible reconstructions of circular extrachromosomal amplicons containing $872 M Y C N$ and ID2 from a single DMG. (D) The simplest possible circle connecting MYCN and ID2. The top track shows copy-number and SVs, the middle track indicates the reconstructed topology, and the bottom track shows 874 H3K27ac binding at the indicated loci in a different pHGG. (E) The chr1 loci connected to the MYCN-ID complex in this DMG. Short-read reconstructions allow for several extrachromosomal amplicons incorporating MYCN-ID2 and

876 MDM4. The difference in the copy number could be explained either by a mix of amplicons containing respectively MYCN-ID2 alone and MYCN-ID2-MDM4 or by more complex amplicons incorporating multiple copies of MYCN878 ID2 for each copy of MDM4. (E) MYCN amplicons in tumors without ID2 amplification (top track) incorporate a larger fraction of the MYCN TAD (60\%) relative to MYCN amplicons in tumors with MYCN-ID2-SV (second track; $88023 \%, p=0.03, T$-test). As a result, the former tend to include more loci with H3K27ac enrichment (third track) from the MYCN-TAD; significantly enriched H3K27ac peaks (q-value <0.01) are indicated with black bars between the 882 H3K27ac fold enrichment and the gene track. (F) MYCN-ID2 amplified pHGGs contain significantly more SVs between the MYCN-TAD and other TADs than pHGGs amplifying only MYCN and its neighborhood. $(p=0.03$, 884 Wilcoxon rank sum test). 


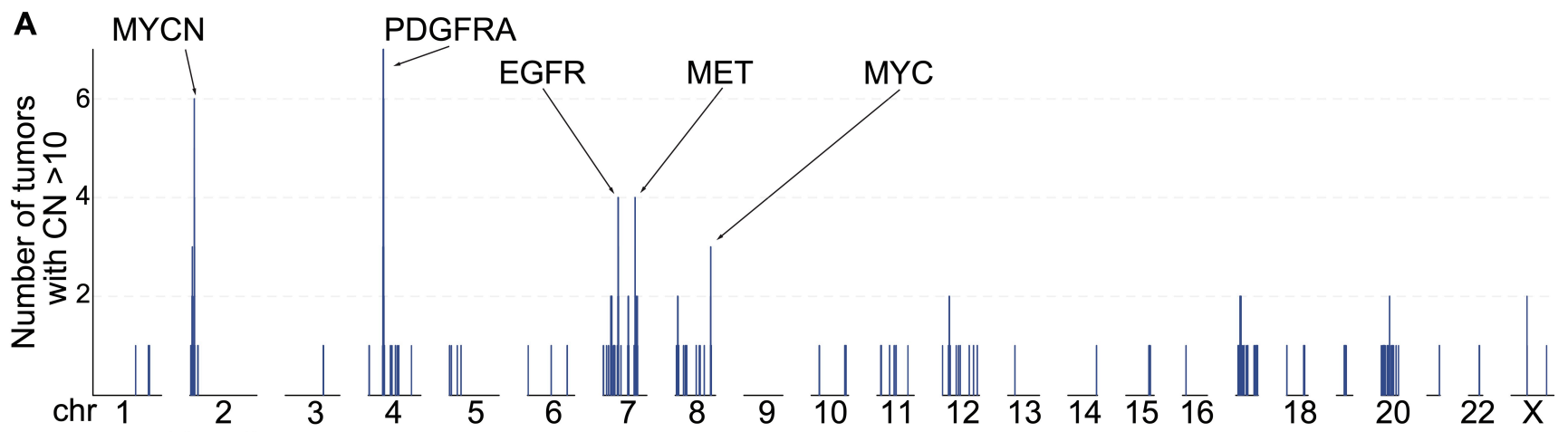
segment location

B simple amplicon including a candidate enhancers

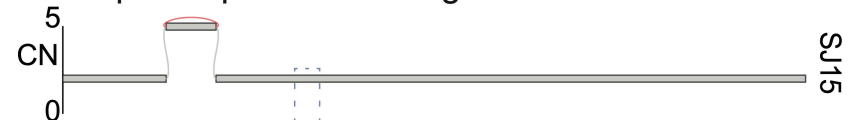

C complex amplicon including PDGFRA exons

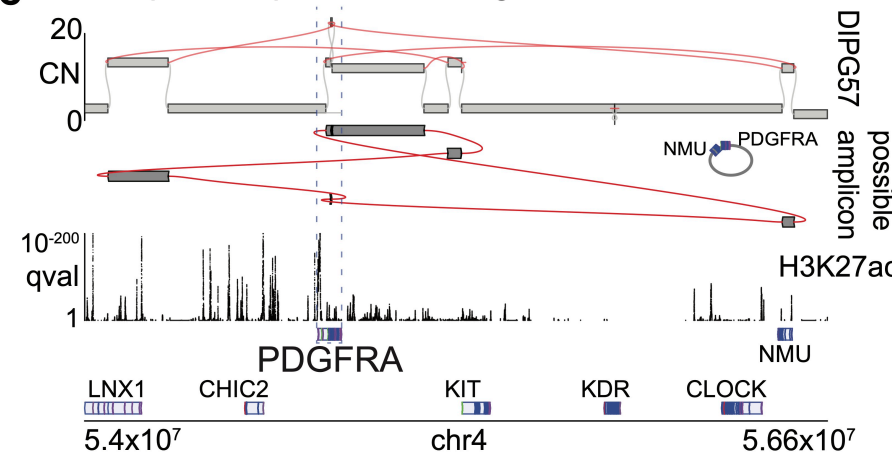

E complex amplicon of EGFR and CCNE1
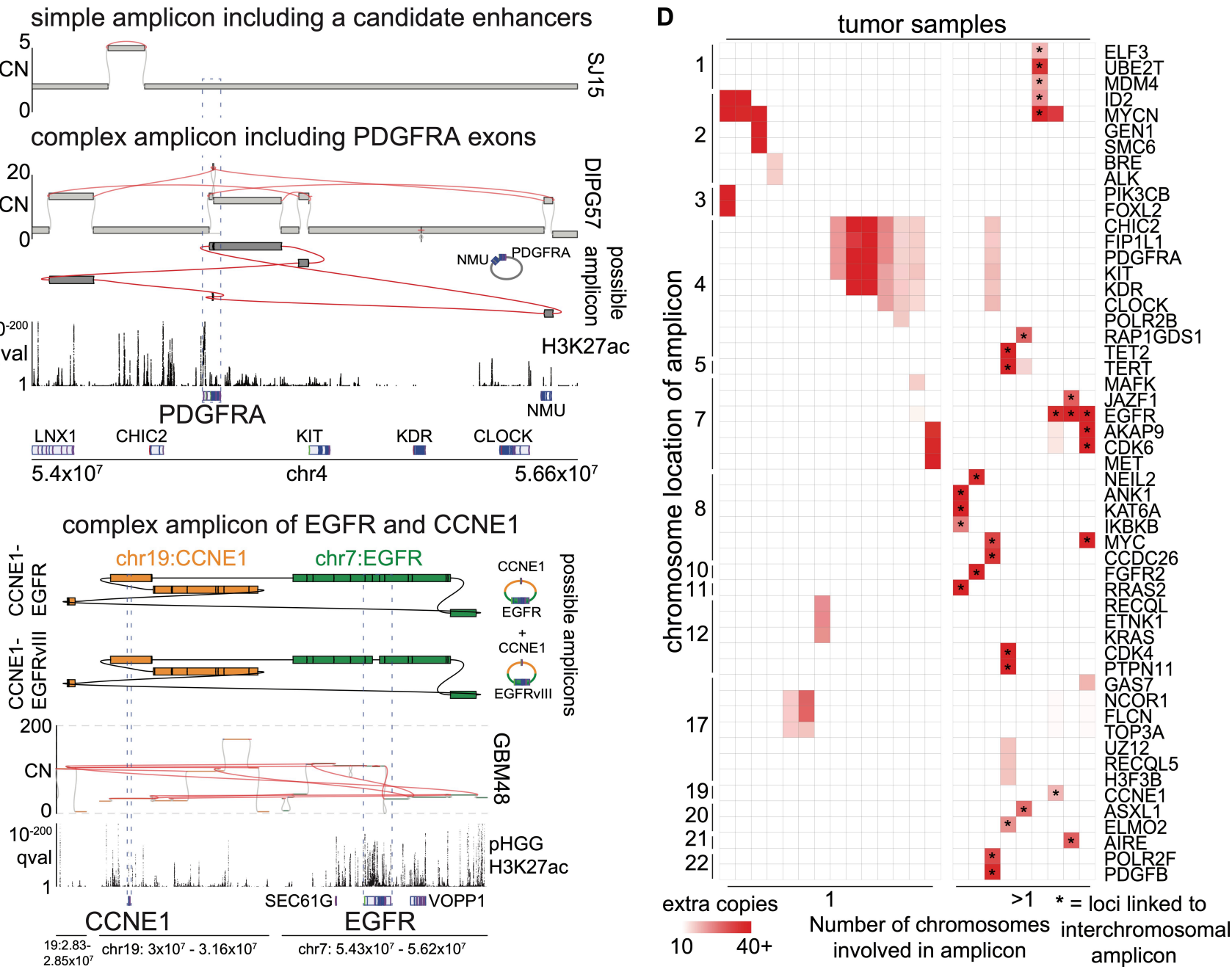

$888 \quad$ Figure 3

High-level amplicons. (A) For each genomic locus (horizontal axis), the number of tumors containing a high-level 890 ( $C N>10$ ) amplicon is indicated (vertical axis). SRBs are highlighted the top. (B-C) Simple and complex-SVs exhibit distinct mechanisms to active PDGFRA. The top and bottom tracks indicate copy-numbers and the significance of 892 H3K27ac enrichment (as calculated from eight pHGGs), respectively. SVs are highlighted in red. Selected gene loci are indicated on the bottom. (B) A simple amplicon of a region with known ${ }^{19,52}$ PDGFRA enhancers. (C) A complex 894 high-level PDGFRA amplicon, displayed as in panel (B) with the addition of a track (second from top) indicating the topology of the amplicon. The complex-SV cluster around PDGFRA connects several segments on chr4, which are amplified to ten absolute copies. The SV calls support the reconstruction of an extrachromosomal amplicon incorporating PDGFRA exons and these segments. (D) Cancer genes involved in high-level amplicons (>10 copies) 
898 within the cohort. 9/23 tumors (grouped on right) contain high-level amplicons encompassing loci from two or more chromosomes. These linked loci are marked by *. The color of each cell represents the number of extra 900 copies due to the amplicon. (E) Example of a tumor with an extrachromosomal amplicon including two oncogenes from different chromosomes. This tumor shows a cluster of SVs connecting the EGFR and CCNE1 loci. The regions 902 of both oncogenes are amplified to different $\mathrm{CNs}$ but in both cases reach several dozen absolute copies. (top) The complexity of the SVs allows for the reconstruction of several possible extrachromosomal amplicons. The CN 904 differences in the bulk profile (middle) could be explained by either a mix of different circles or by more complex circles incorporating some segments repeatedly. The SV calls also reveal that a small fraction of the EGFR 906 amplicons in this patient already show the EGFRvIll variant. The bottom two tracks show the genes of interest at the location and q-value H3K27ac track calculated from eight pHGG tumors. 
A
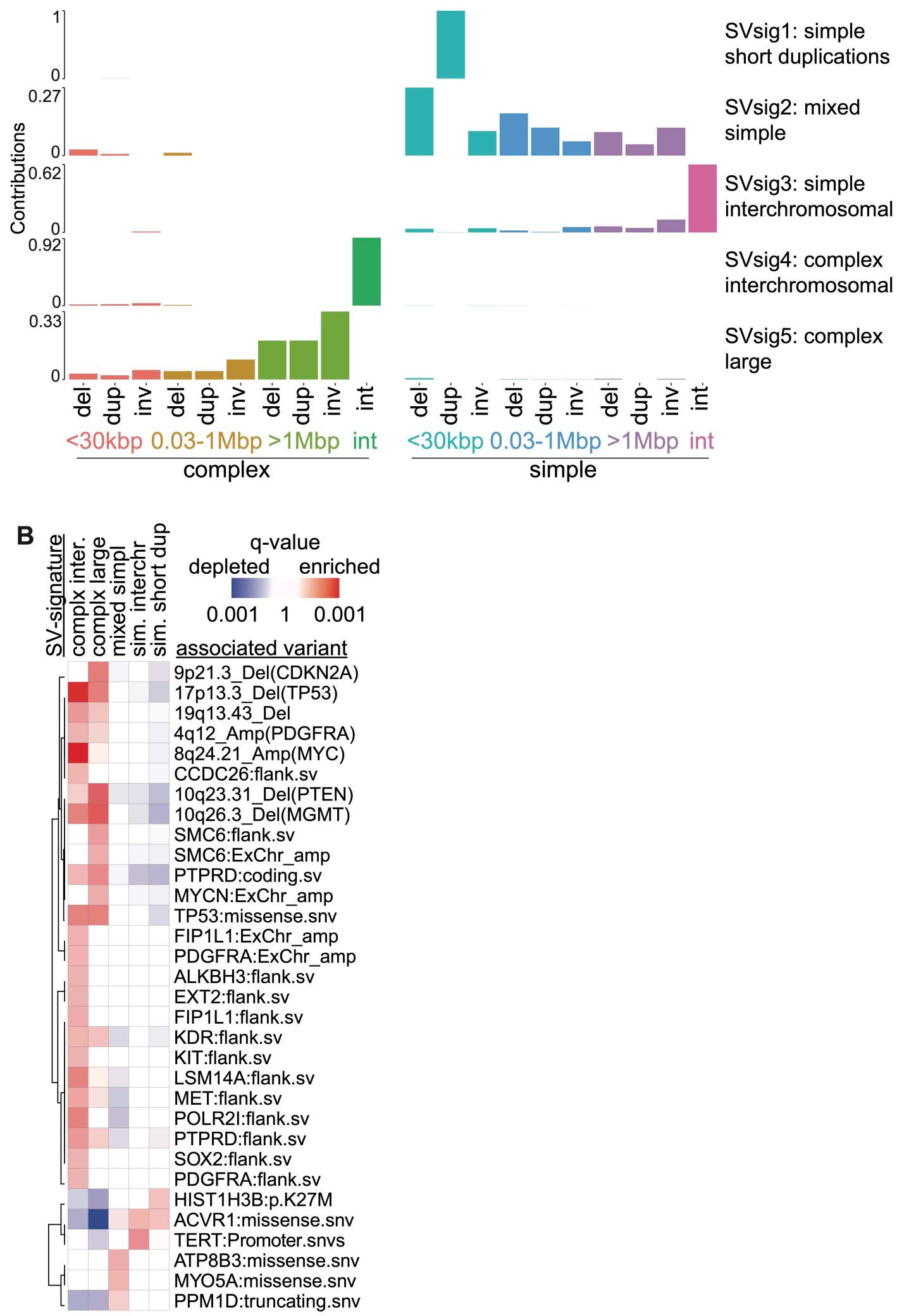

Figure 4

SV signatures in pHGG. (A) The horizontal axis indicates the size and type of SVs. Del stands for deletion, dup for 912 duplication, inv for inversion, and int for interchromosomal rearrangement. The vertical axis indicates the fraction of SVs within each signature that are contributed by each SV type. (B) The statistical significance of 
914 positive (enriched) and negative (depleted) associations between each SV signature and of all recurrently altered somatic genetic alterations that are documented in the Cancer Gene Census ${ }^{53}$. 
A

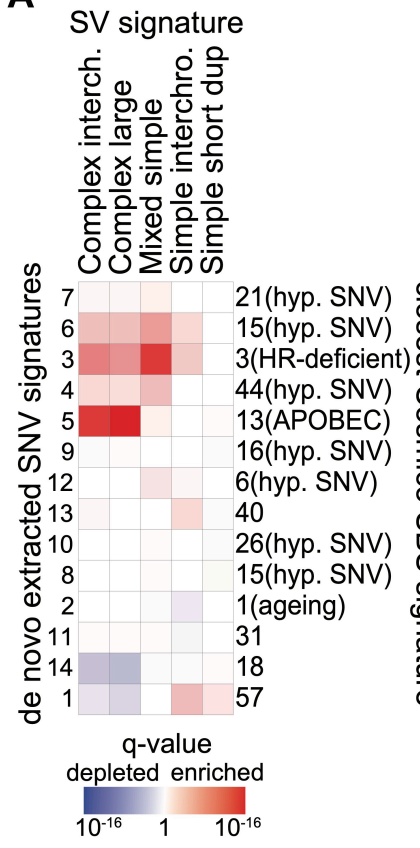

C

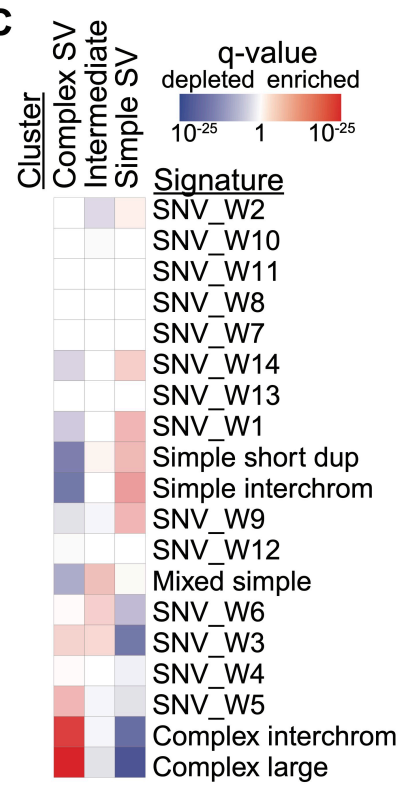

Complex SV

Intermediate

Simple SV

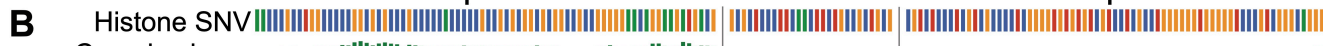
Complex large

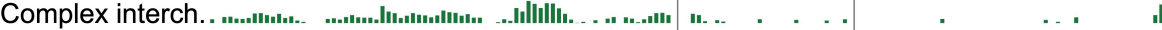

Simple interchro. $*$

Simple short dup

Mixed simple -............

"SBS3 HRdef"

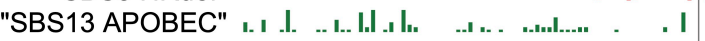
SNV9/"MSI"

ACVR1 1

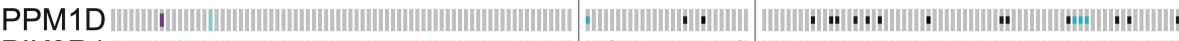

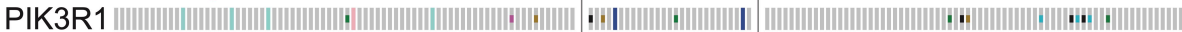
PIK3CA

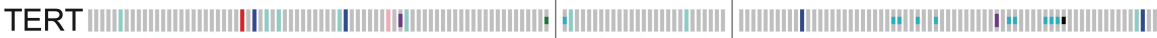

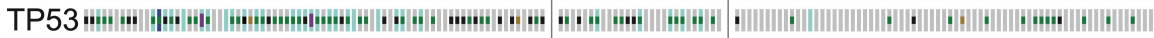
PDGFRA

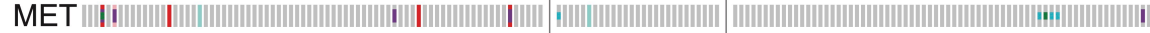

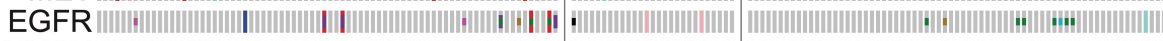
MYCN

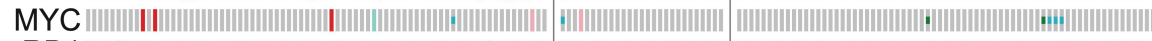

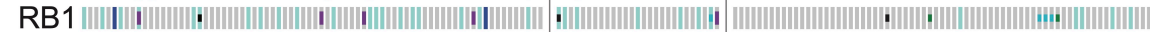

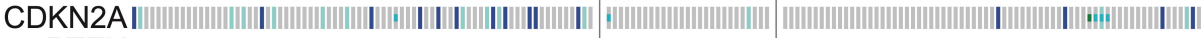

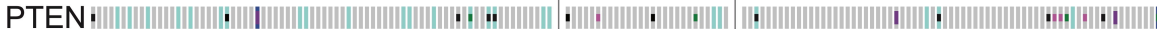

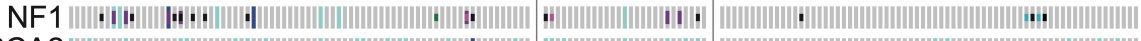
BRCA2

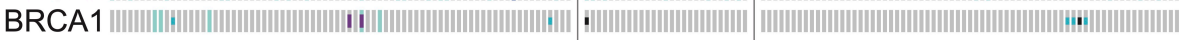
Histone SNV H3.1K27M H3.3K27M |H3.3G34R |WT

Genetic alteration

- Missense mutation - Truncating mutation

- Promoter mutation =Other mutation Inframe mutation (unknown significance)

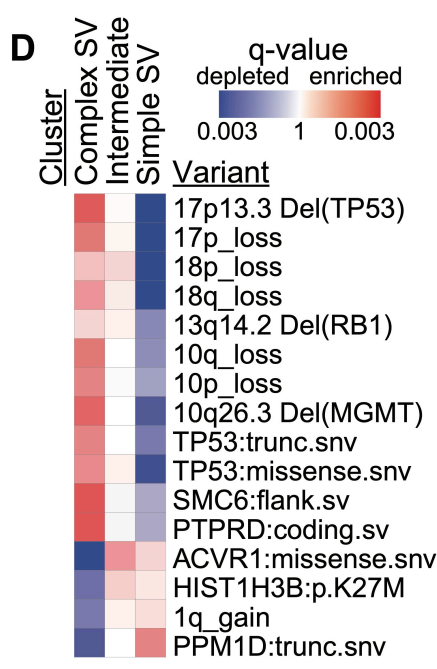

I Coding SV $\mid \mathrm{CN}>10 \quad \mathrm{CN}>4.2$

| $\mathrm{CN}<0.4 \quad \mathrm{CN}<1.2 \quad$ No alterations

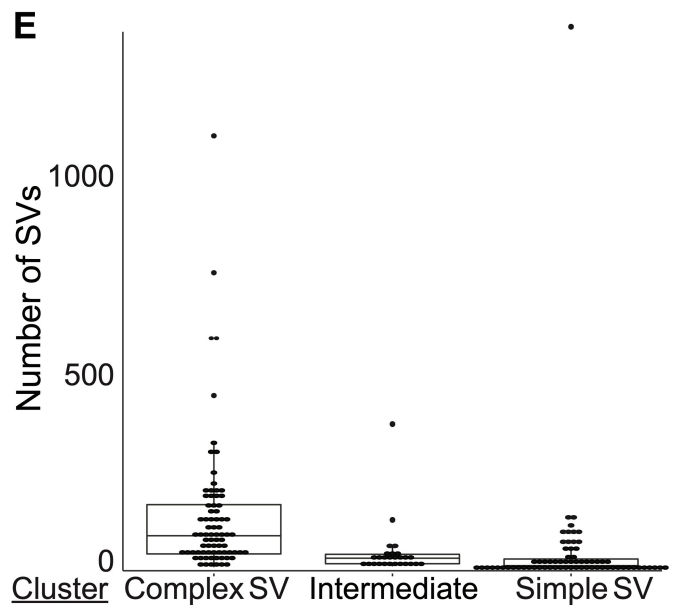

$918 \quad$ Figure 5

Integrated SV and SNV signature analyses reveal three pHGG subgroups with distinct variant generating processes. (A) Correlations between SV and SNV signatures. Signature labels from this analysis are indicated on the left; the nearest COSMICv3 signatures are indicated on the right, with their proposed mechanisms in parentheses. Complex-SV signatures show a close correlation with APOBEC and homologous recombination deficiency SNV signatures (SBS3). q-values are based on Spearman rank correlations. (B) Consensus clustering of the normalized SNV and SV signature activities in each tumor sample (columns). Rows indicate signature activities (top) and potentially oncogenic variants (bottom). (C) Enrichment analysis for signature activities in each cluster from panel B. FDR q-values are based on Wilcoxon tests. (D) Significance of signature cluster associations for all variants with correlations reaching $q<0.1$; q-values are based on Fisher's exact tests. Tumors in the complex-SV clusters are enriched for copy-number changes in cancer genes and SNVs in TP53, whereas simple-SV pHGGs tend 
to exhibit SNVs in different cancer genes. (E) Number of SVs per tumor in each cluster. All differences are 930 significant to $\mathrm{q}<0.003$ by Wilcoxon tests. 
Complex SV sig

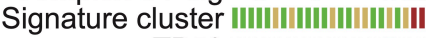

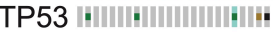

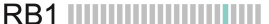

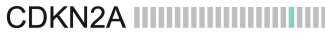

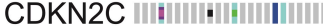

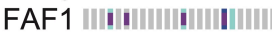

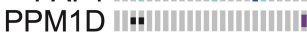

MDM4

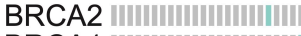

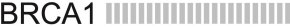

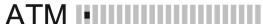

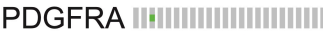

ACVR1

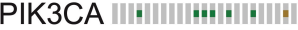

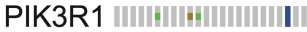

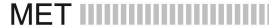

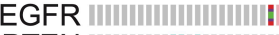

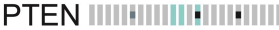

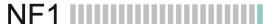

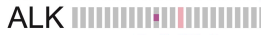

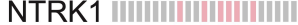

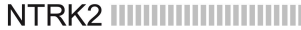

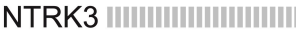

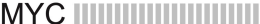

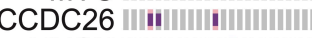

MYCN

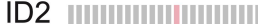

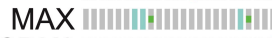

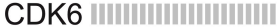

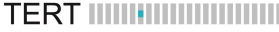

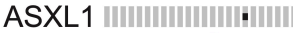

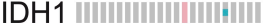

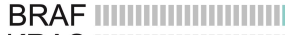

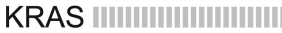

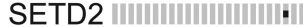

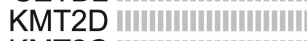

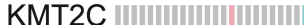

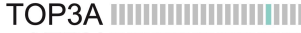

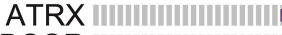

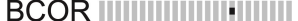

Location "n!"

Signature cluster
| Complex SV
Simple SV Intermediate
NA

B 20 q-value

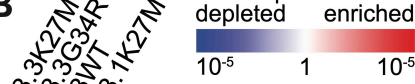

(a) Genetic alteration 2p25.1 Amp

2p25.1 Amp(KIDINS220)

$2 p$ gain

2q gain

2p24.3 Amp(MYCN)

HIST1H3C missense.snv

$7 p$ gain

HIST1H3Bp.K27M

1q gain

FAF1 coding.sv

ACVR1 missense.snv

7p11.2 Amp(EGFR)

7p15.1 Amp

CDKN2B homdel

CDKN2A homdel

7q21.2 Amp(CDK6)

ATRXtruncating.snv

H3F3Amissense.snv

$11 \mathrm{ploss}$

17p13.3 Del(TP53)

$17 \mathrm{p}$ loss

8q24.21 Amp (CCDC26)

ASXL1 truncating.snv

KITflank.sv

FIP1L1 flank.sv

H3F3Ap.K27M

TP53 missense.snv

4q35.1 Del

932

TP53 truncating.snv

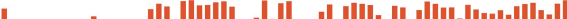

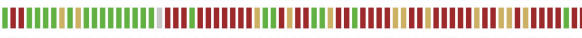
อง

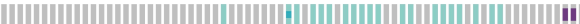

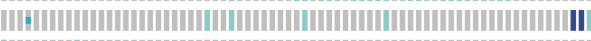
|

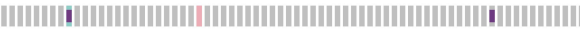

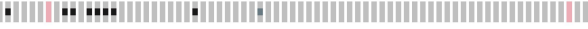

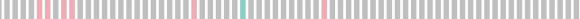
|

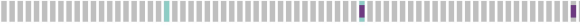

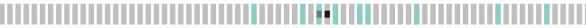

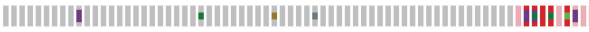
(1)

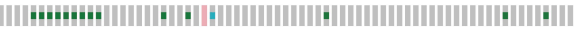

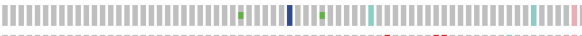
1

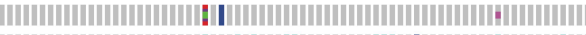

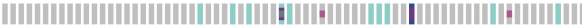

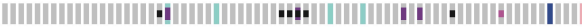

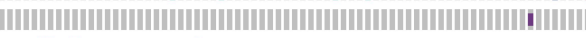
П.

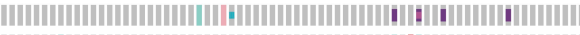

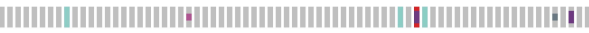

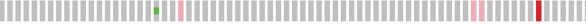

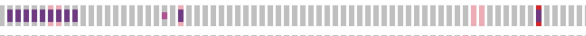

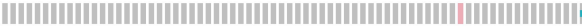

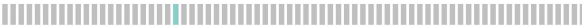
ป .

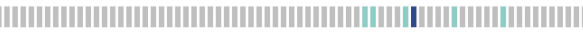
ค. : О) | |กัก

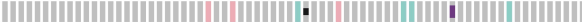

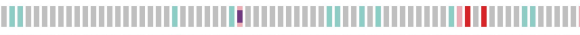
| 4 (ด)

Genetic alteration
- Missense mutation (putative driver)
Missense mutation (putative driver) - Truncating mutation (putative driver) Iruncating mutation (unknown significance)

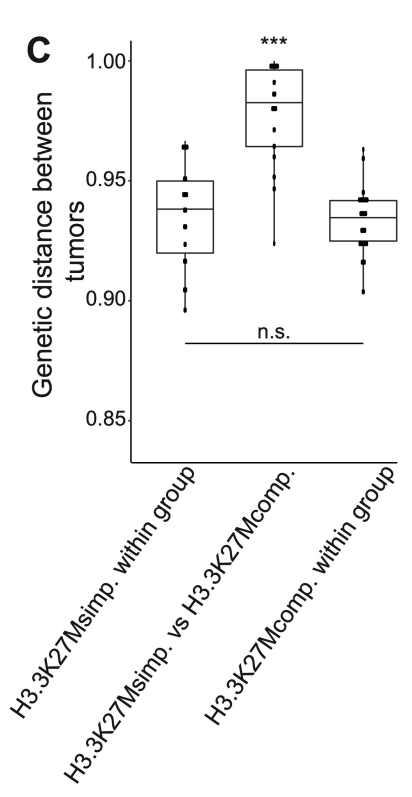

H3.3G34R

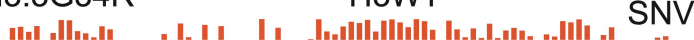

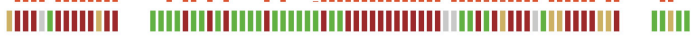

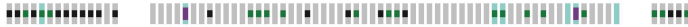
.

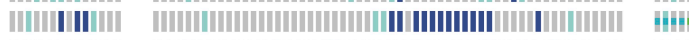

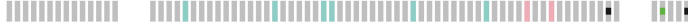

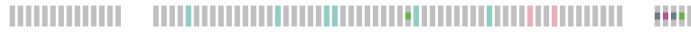
|

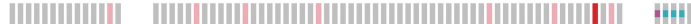

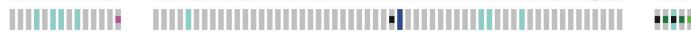

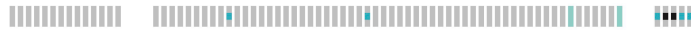

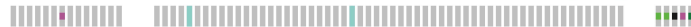
|

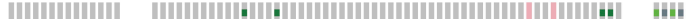

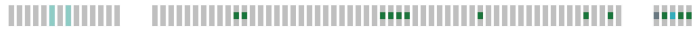

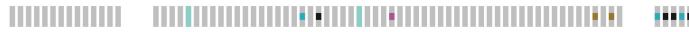

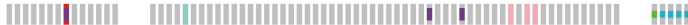

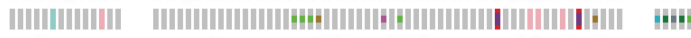

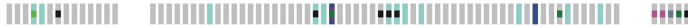

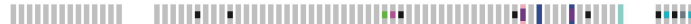
| 1

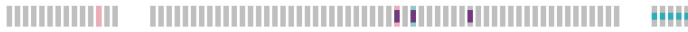

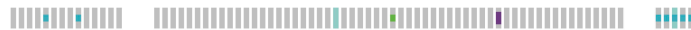
1 I

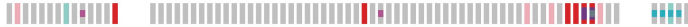

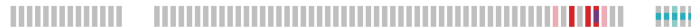
|

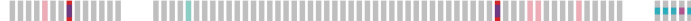

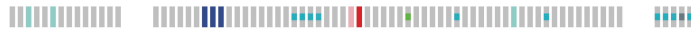

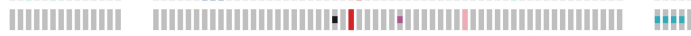

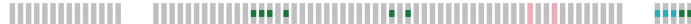

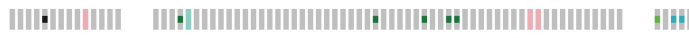
| |

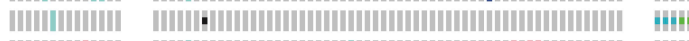

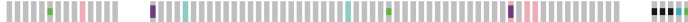
|

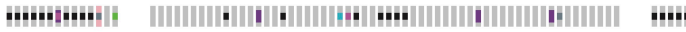

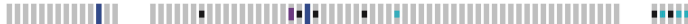

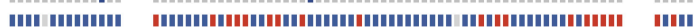
Promoter mutation Other mutation Location

Inframe mutation (unknown significance) IHemispheric

\begin{tabular}{l|l|l|l} 
Coding SV & $\mathrm{CN}>10$ & $\mathrm{CN}>3.4$ & Midline
\end{tabular}

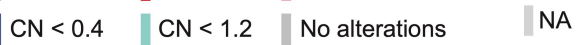

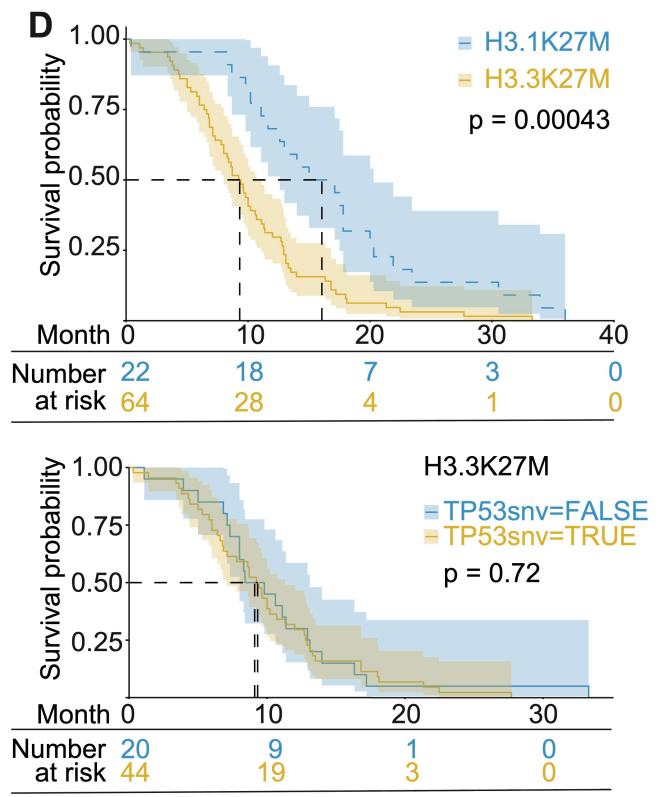

Figure 6 
Overview of somatic variants and associated features within histone mutation-defined subgroups. (A) Co-mut 936 plot of the $176 / 179$ (98.3\%) tumors with somatic variants in at least one well-known oncogene. Columns represent tumors, ordered within histone mutation-defined subgroups by hierarchical clustering of all potential driver 938 variants. The top two rows show signature metadata. (B) Significance of histone subgroup associations for all variants with correlations reaching $q<0.05$ (based on Fisher's exact tests) within any subgroup. (C) Jaccard 940 distances based upon the variants in each tumor (vertical axis), for pairs of tumors within the H3.3 ${ }^{\mathrm{K} 27 \mathrm{M}}$ Simple-SV group (left column), within the H3.3 ${ }^{\mathrm{K} 27 \mathrm{M}}$ Complex-SV group (right column), or paired between these groups (middle 942 column). H3.3 ${ }^{\mathrm{K} 27 \mathrm{M}}$ tumors were considered to be in the Complex-SV group if Complex-SV signatures comprised more than $20 \%$ of its SV signature activity. ${ }^{* * *}$ denotes $\mathrm{q}<6 \times 10^{-8}$ relative to both other columns by a Wilcoxon 944 test. (D) Kaplan-Maier plot indicating overall survival for (top) H3.1 ${ }^{\mathrm{K} 27 \mathrm{M}}$ and H3.3 ${ }^{\mathrm{K} 27 \mathrm{M}}$ DMGs and (bottom) H3.3 ${ }^{\mathrm{K} 27 \mathrm{M}}$ DMG with and without TP53 SNVs. p-values are from log-rank tests. 
A

Focal TAD amplification excluding oncogene

"TAD-Amp"

Histone SNV

Signat. cluster

Complex SV sig

CCDC26

MYC

MYCN

ID2

PDGFRA

EGFR

MET

TP53

RB1

CDKN2A

CDKN2C

FAF1

PPM1D

MDM4

Location

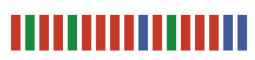

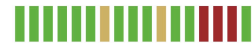

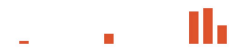

IIIII II III

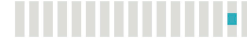

प| ||||||||||||||||
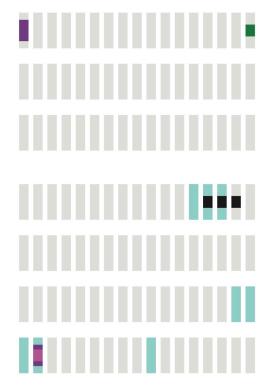

II

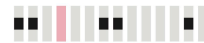

IIII

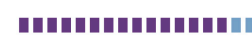

TAD-Amp
High level oncogene amplification (>3.4 copies)

"Onc-Amp"

| ||||||||||||||||||||||||||||||||||||| | | ||||||||||||||||||||||||||||||||| || |

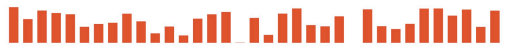

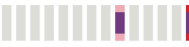

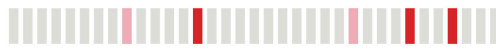
IIIIII III

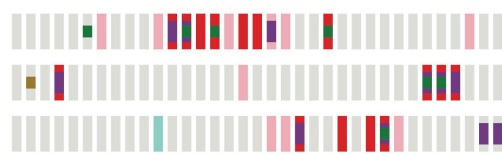

4|||

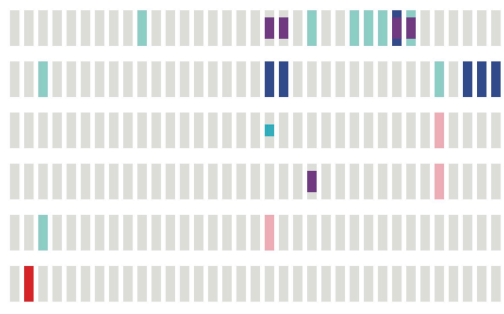

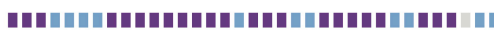

Onc-Amp
Histone SNV

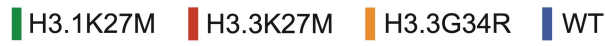

Signature cluster

Icomplex SV intermediate simple SV

Complex SV signature activity

$0 \% \quad 100 \%$

Genetic alteration

- Missense SNV "Truncating SNV Promoter SNV

I Other SNV I Coding SV No alterat.

- Inframe SNV (unkn.sign.)

| $\mathrm{CN}>10|\mathrm{CN}>3.4| \mathrm{CN}<0.4 \mid \mathrm{CN}<1.2$

Location

Hemispheric Midline NA

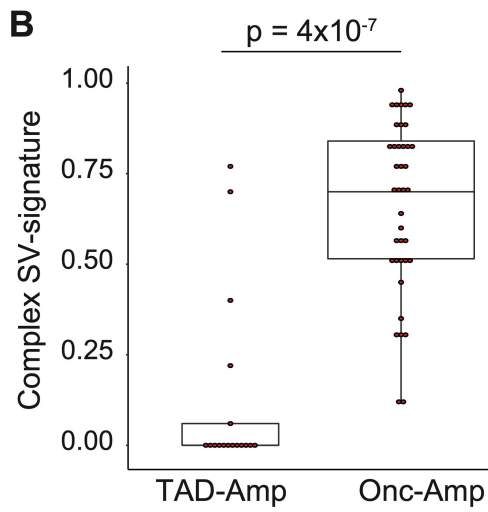

$\begin{array}{rrrr}\text { PPM1D:truncating.snv } & 29 \% & \mathrm{q}=0.03 & 0 \% \\ \text { 1q /MDM4 gain } & 71 \% & \mathrm{q}=0.16 & 34 \% \\ \text { 13q14.2Del(RB1) } & 0 \% & \mathrm{q}=0.16 & 23 \% \\ \text { TP53:SNV } & 18 \% & \mathrm{q}=0.01 & 69 \%\end{array}$

C

8q24.21(CCDC26) Amp

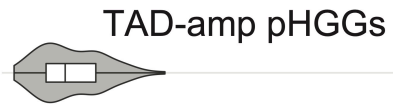

1q gain

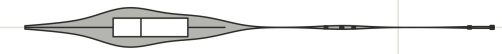

TP53 snv

PPM1D snv

PIK3CA snv

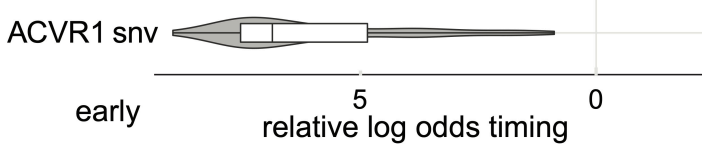

D 8q24.21(CCDC26) Amp 7q31.2(MET) Amp 7q21.2(CDK6) Amp 7p11.2(EGFR) Amp 4q12(PDGFRA) Amp 2p24.3(MYCN) Amp 9p21.3(CDKN2A) Del 17p13.3(TP53) Del 13q14.2(RB1) Del

TP53 snv PIK3CA snv PDGFRA snv EGFR snv early

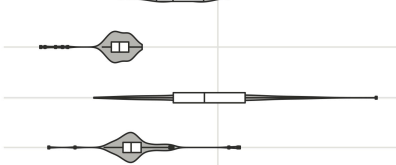

Onc-amp pHGGs
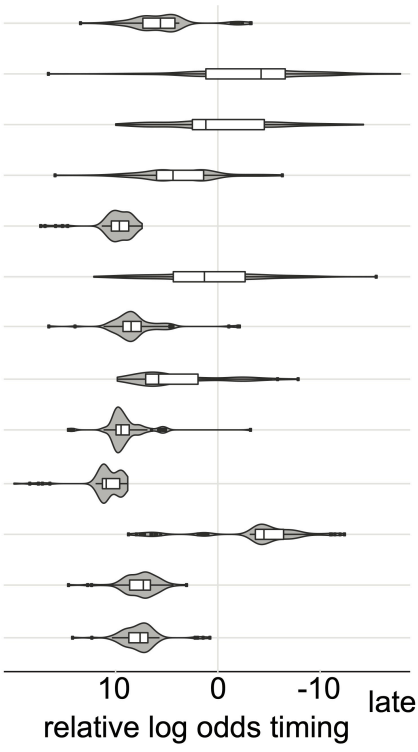

Figure 7

948 Context of the significantly recurrent SVs. (SRSVs) (A) Co-mut plot for TAD-amp and Onc-amp SRSVs. TAD-amp SRSVs generate focal amplifications in the TAD of an oncogene without amplifying the protein coding sequence; 950 Onc-amp SRSVs generate high-level ( $C N>3.4)$ amplifications or fusions of the coding sequence. The top three rows show associated metadata. The next seven rows indicate the genes affected by the SRSVs. The bottom seven rows 952 show genes in DNA damage response pathways. Significant associations with the two groups are illustrated with pie charts below the plot, based on Fisher's exact tests. (B) TAD-amp pHGGs show significantly lower combined 
954 Complex-SV signature activity than Onc-amp pHGGs ( $p=4 \times 10^{-7}$, Wilcoxon). (C-D) Timing analysis of somatic variant acquisition in TAD-amp (C) and Onc-amp (D) pHGGs based on a Bradley-Terry model. The horizontal axis shows 956 the log odds of the variant being an early event. The distributions indicate the results of 100 random subsamples of the data. Only variants in the SRSV-affected genes and pathways (growth factor and MYC signaling) and in DNA 958 damage response genes altered in more than two samples are shown. 


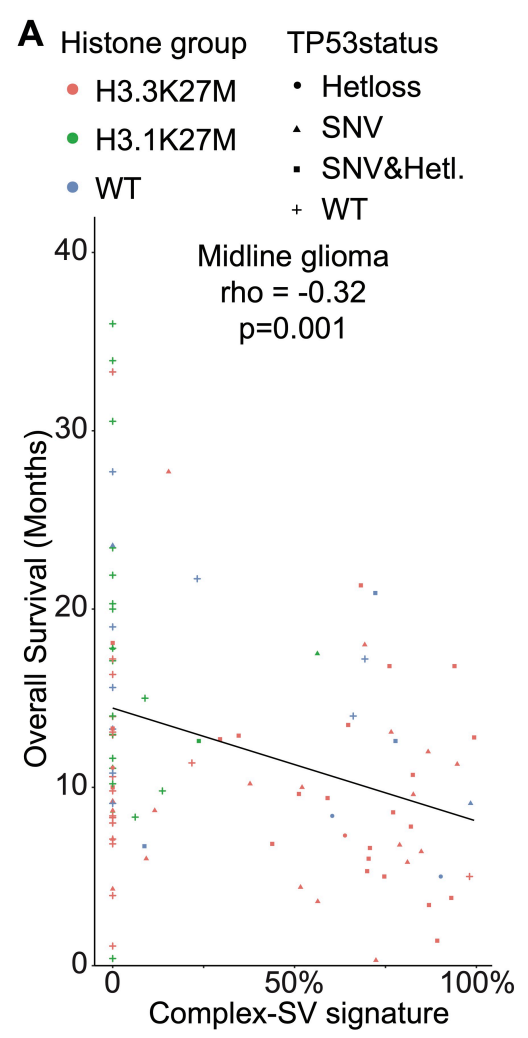

B

Hazard ratio

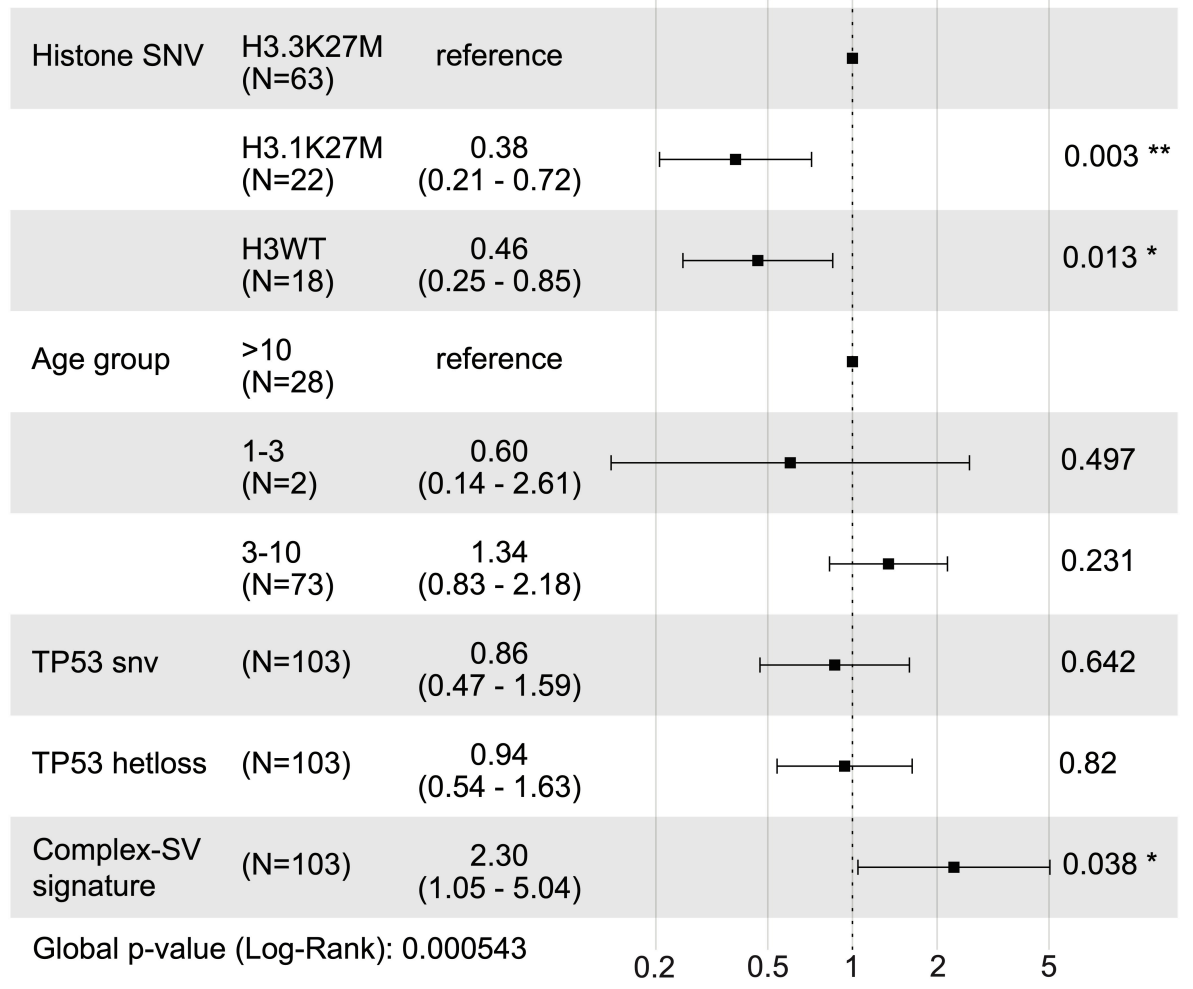

C

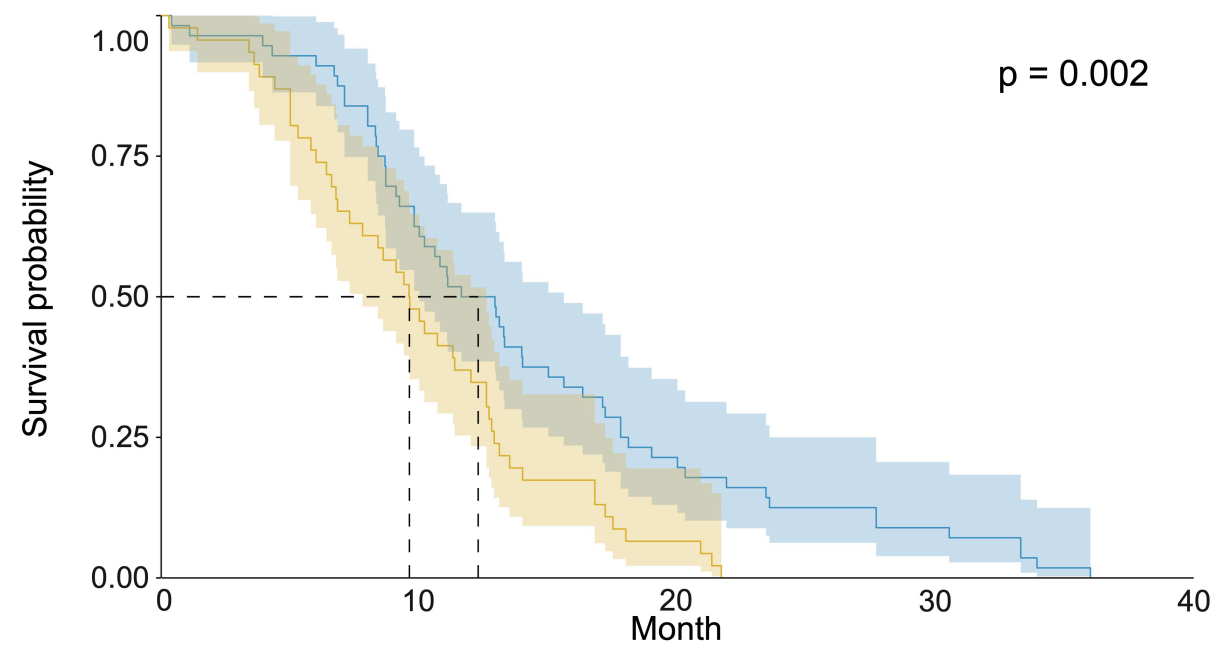

Complex-SV Number at risk

sign. $<20 \% \quad 56$

Complex-SV

sign. $>20 \%$

960

Figure 8

962 Association between the complex-SV signature and overall survival in midline gliomas. (A) Associations between the fraction of SV signature activity attributed to Complex-SV signatures and overall survival for all midline gliomas $(n=103 ;$ rho $=-0.32$ and $p=0.001$, Spearman correlation). (B) Cox proportional hazards analysis incorporating histone group, age, TP53 status and the combined Complex-SV signature. (C) Kaplan-Maier plot of a univariate analysis of the association between the Complex-SV signature and overall survival, in which tumors were classified as Complex-SV if Complex-SV signatures contributed more than $20 \%$ of all SV signature activity. The number of patients in each group is indicated below the plot. The p-value represents a log-rank test. 
Extended Data Figure 1

970 Sample characteristics and significantly recurrent variants. (A) Purity of pre-treatment biopsy and autopsy samples were not significantly different ( $p=0.5$, Wilcoxon). (B) Significantly recurrent SNVs in non-hypermutant 972 tumors. (C) Significantly recurrent SCNAs. All of these SCNAs have been noted ${ }^{3}$ except for a novel non-proteincoding locus in 8q.24.21, near MYC-which is also within a separate recurrently amplified locus. (D) Q-Q plot for

974 the analysis of significantly recurrent SV breakpoints. The most significantly recurrent breakpoints are within the long non-coding RNA CCDC26, within the TAD encompassing MYC.

976

\section{Extended Data Figure 2}

978 Lineage specificity of the enhancer peak in CCDC26. (A) ATAC-seq (top) and H3K27ac ChIP-seq (bottom) enrichment (vertical axis) of samples from different lineages (indicated on right; "27y" indicates the sample was 980 obtained from a 27-year old person) across the TAD encompassing MYC (horizontal axis). The location of the MYC coding sequence is highlighted in red. The CCDC26 amplicon boundaries for the 15 samples with the amplicon are indicated by the paired red arrows at the top. The consensus amplicon is indicated by the green dotted lines and centers on an H3K27ac peak present only in glial samples. (B) Hi-C heatmaps depicting DNA interaction profiles (5 $\mathrm{kb}$ resolution) from iPSC-derived neural progenitor cells (top) and two cell lines harboring $\mathrm{H} 3.3^{\mathrm{K} 27 \mathrm{M}}$ mutations (middle and bottom). Red and white indicate high and low interaction frequencies, respectively. MYC interacts more frequently with the H3K27ac peak within CCDC26 (black oval) relative to neighboring loci. The minimal common region of the CCDC26 amplicon is indicated at the bottom of the heatmaps (SV MCR; blue rectangle).

\section{Extended Data Figure 3}

990 Significantly recurrent juxtaposition between MYCN and ID2 (A) (top) Count matrix showing all possible juxtapositions between pairs of genomic loci. (bottom) Illustration of the principle behind the analysis of recurrent juxtapositions, as exemplified by the MYCN-ID2 loci. First, we count the number of SVs connecting each pair of genomic loci. Using a background model for the probability of juxtapositions generated from an analysis of 2658 cancers $^{22}$, we then determine the probability of observing this number of SVs due to chance alone, corrected for multiple hypothesis testing. This analysis revealed the MYCN-ID2 juxtaposition as the only significantly recurrent juxtaposition in the window shown. (B) Overlay of amplification frequencies on ChIP-seq data in the ID2 and MYCN loci. The top two tracks show, among tumors with $M Y C N$-ID2 rearrangements (top track) or MYCN amplifications without ID2 involvement (second track), the percentage of tumors with amplifications ( $y$-axis) at each genomic locus (x-axis). The bottom eight tracks indicate H3K27ac ChIP-seq profiles across these loci for four H3 ${ }^{\mathrm{K} 27 \mathrm{M}}$ and four $\mathrm{H}^{\mathrm{WT}}$ pHGGs tumors. Coding sequences of ID2 and MYCN are highlighted with yellow and red lines respectively. Significantly enriched H3K27ac peaks (q-value $<0.01$ ) are indicated below each ChIP-seq track. The small region at the ID2 locus that is amplified in all MYCN-ID2 pHGGs shows an H3K27ac peak in the ChIP tracks from all six pHGG tumor samples. Tumors that amplify MYCN without ID2 take in a much larger region of the MYCN TAD into the amplicon (60\% vs. $23 \%, p=0.03$, T-test). (C) G-track plots indicating copy-number profiles and genome topology after consideration of local SVs, for two examples of pHGGs with focal MYCN amplicons without incorporation of ID2. For both tumors the copy number and SV profiles support several possible reconstructions of extrachromosomal circular amplicons. All are limited to the neighborhood of $M Y C N$, presumably incorporating endogenous enhancers from the MYCN TAD.

\section{Extended Data Figure 4}

Structures of recurrent RTK amplicons (A) Average amplicon profile for all pHGGs with amplifications in the 
1014

1016

1018

1020

1022

1024

1026

1028

1030

1032

1034

1036

1038

1040

1042

1044

1046

1048

1050

1052

1054

1056

tumors with amplifications (vertical axis) at each location (horizontal axis). The track below shows the average $\mathrm{CN}$ across all tumors with amplifications in the PDGFRA TAD. The segments included in the PDGFRA amplicon in 80\% of tumors are highlighted in the red box. Most amplicons range over several Mbp, often including KIT. (B) Average amplicon profile for all pHGGs with amplifications in the EGFR TAD reaching at least four copies $(n=7)$, displayed as in (A). The segments included in all the EGFR amplicons are highlighted in the red box. The pHGG EGFR amplicons always include upstream enhancers elements around SEC61G known to activate EGFR in adult $\mathrm{GBM}^{21}$. (C) Structure of a simple EGFR-TAD amplicon that encompasses enhancers that are also amplified in all tumors with EGFR coding sequence amplifications. (D) SVs, CN tracks and reconstructions for all pHGGs with high-level MET amplifications. The observed high-level MET amplicons are a few $100 \mathrm{kbp}$ in size. Three out of four METamplified pHGGs incorporate a downstream region including an enhancer (see bottom H3K27ac track) into the amplicon. For all four MET-amplified pHGGs possible reconstructions of the extrachromosomal amplicon are shown above the $\mathrm{CN}$ and SV track. (A-D) From bottom to top the tracks show: the genes of interest at the location, a q-value H3K27ac track calculated from eight pHGG tumor samples, the CN and SV for the indicated tumor at the location and reconstructions of possible extrachromosomal amplicons if applicable.

\section{Extended Data Figure 5}

Associations between SV-defined groups. (A) Jaccard distances between tumor pairs (vertical axis), calculated from the combination of variants in each tumor, across subgroups of $\mathrm{H}^{\mathrm{K} 27 \mathrm{M}}$ DMGs. Tumor groups were determined by whether mutations were present in H3.1 or H3.3 and whether the combined Complex-SV signatures contributed more or less than $20 \%$ to all SV signature activity. Tumors were paired within or between these groups, as indicated on the horizontal axis. All differences were significant with $q<0.005$ (FDR-corrected Wilcoxon test) unless indicated otherwise. (B) Volcano plot indicating the significance (vertical axis; FDR-corrected Fisher's exact tests) of associations in $\mathrm{H} 3.3^{\mathrm{K} 27 \mathrm{M}}$ Simple-SV relative to $\mathrm{H} 3.3^{\mathrm{K} 27 \mathrm{M}}$ Complex-SV DMGs, for all genetic variants observed in at least $10 \%$ of tumors in one subgroup. Variants enriched or depleted to $\mathrm{q}<0.15$ are highlighted in red on the right or in blue on the left, respectively. $\mathrm{H} 3^{\mathrm{K} 27 \mathrm{M}}$ DMGs were considered to be in the Complex-SV group if Complex-SV signatures comprised more than $20 \%$ of its SV signature activity. (C) Association between MDM4 expression (vertical axis) and copy number (horizontal axis). MDM4 gains universally represent arm-level gains of $1 q . * * *$ indicates adjusted $p=0.004$, ANOVA with Tuckey post-test. (D-E) Volcano plot indicating the significance (vertical axis; FDR-corrected Fisher's exact tests) of associations between genetic variants and pHGG subgroups (horizontal axis), colored as in B. (E) Enrichment or depletion of arm-level SCNAs in TP53disrupted ( $n=97$ ) vs TP53 ${ }^{\text {WT }}(n=77$ ) pHGGs. We considered samples to have TP53 disruption if the gene exhibited SNVs ( $n=88$, often with copy loss as well) or copy loss alone ( $n=9)$. (F) Enrichment or depletion of arm-level SCNAs in TP53-disrupted ( $n=56$ ) vs TP53 ${ }^{\mathrm{WT}}(\mathrm{n}=39) \mathrm{H} 3^{\mathrm{K} 27 \mathrm{M}}$ mutant DMGs. TP53 disruption represented SNVs (often with copy loss) in 53 samples or copy loss alone in 3 samples. Only significantly recurrent arm-level SCNAs are shown.

\section{Extended Data Figure 6}

Timing analysis of somatic variant acquisition in histone mutation-defined pHGG subgroups. For each subgroup the individual (per-sample) timing of recurrent variants is fed into a Bradley-Terry model. This results in a strength parameter for each variant which is indicated on the $\mathrm{x}$-axis in log scale and can be interpreted as the relative log odds of the variant being an early event in this subgroup. Each distribution indicates the results of 100 random subsamples of the respective subgroup. Only potential driver variants recurrent in more than two samples are shown. Subgroups: (A) H3.1 $1^{\mathrm{K} 27 \mathrm{M}}$ (B) H3.3 $3^{\mathrm{K} 2 \mathrm{MM}}$ (C) H3.3G34R (D) H3 ${ }^{\mathrm{WT}}$ (E) hypermutant pHGGs.

\section{Supp. Fig. 1}


1060 Supp. Fig. 2

SV signature activities across all samples in the cohort. (A) SV signature activity in every tumor. Tumors with 1062 higher SV counts on the left show complex-SV signature activity whereas tumors with lower SV counts on the right show a mix of simple-SV signatures.

1064

\section{Supp. Fig. 3}

1066 SNV signature activities across all samples in the cohort (A) Cosine similarity between de novo extracted SNV signatures and the COSMICV3 SBS-signatures. (B) SNV signature activity in every tumor. The hypermutant tumors 1068 on the left show signatures associated with hypermutation in COSMICv3. Signature 3, which is similar to the SBS3 homologous recombination deficiency signature, features prominently in many non-hypermutant tumors.

\section{Supp. Fig. 4}

1072 Comparison between pre-treatment biopsy and autopsy samples. These groups exhibit no significant differences in (A) the number of SVs per sample ( $q=0.6$, Wilcoxon) or $(\mathbf{B})$ the activity of the combined complex-SV signatures 1074 ( $q=0.7$, Wilcoxon). 
References

1076

1078 1. Ostrom QT, Gittleman H, Truitt G, Boscia A, Kruchko C, Barnholtz-Sloan JS. CBTRUS Statistical Report: Primary Brain and Other Central Nervous System Tumors Diagnosed in the United States in 2011-2015.

$1080 \quad$ Neuro Oncol. 2018;20(suppl_4):iv1-iv86. doi:10.1093/neuonc/noy131

2. Puget S, Philippe C, Bax DA, et al. Mesenchymal Transition and PDGFRA Amplification/Mutation Are Key

1082 Distinct Oncogenic Events in Pediatric Diffuse Intrinsic Pontine Gliomas. PLoS One. 2012;7(2):e30313. https://doi.org/10.1371/journal.pone.0030313.

1084 3. Mackay A, Burford A, Carvalho D, et al. Integrated Molecular Meta-Analysis of 1,000 Pediatric High-Grade and Diffuse Intrinsic Pontine Glioma. Cancer Cell. 2017;32(4):520-537.e5.

1086 doi:https://doi.org/10.1016/j.ccell.2017.08.017

4. Project ICGCPT, Bender S, Gronych J, et al. Recurrent MET fusion genes represent a drug target in 1088 pediatric glioblastoma. Nat Med. 2016;22:1314. https://doi.org/10.1038/nm.4204.

5. Buczkowicz P, Hoeman C, Rakopoulos P, et al. Genomic analysis of diffuse intrinsic pontine gliomas 1090 identifies three molecular subgroups and recurrent activating ACVR1 mutations. Nat Genet. 2014;46(5):451-456. doi:10.1038/ng.2936

6. Wu G, Diaz AK, Paugh BS, et al. The genomic landscape of diffuse intrinsic pontine glioma and pediatric non-brainstem high-grade glioma. Nat Genet. 2014;46(5):444-450. doi:10.1038/ng.2938

7. Taylor KR, Mackay A, Truffaux N, et al. Recurrent activating ACVR1 mutations in diffuse intrinsic pontine glioma. Nat Genet. 2014;46(5):457-461. doi:10.1038/ng.2925

1096 8. Project SJCRHUPCG, Wu G, Broniscer A, et al. Somatic histone H3 alterations in pediatric diffuse intrinsic pontine gliomas and non-brainstem glioblastomas. Nat Genet. 2012;44:251. https://doi.org/10.1038/ng.1102.

9. Schwartzentruber J, Korshunov A, Liu X-Y, et al. Driver mutations in histone H3.3 and chromatin remodelling genes in paediatric glioblastoma. Nature. 2012;482:226. https://doi.org/10.1038/nature10833.

10. Bigner SH, Wong AJ, Mark J, et al. Relationship between gene amplification and chromosomal deviations in malignant human gliomas. Cancer Genet Cytogenet. 1987;29(1):165-170. doi:10.1016/01654608(87)90045-8

11. Shoshani O, Brunner SF, Yaeger R, et al. Chromothripsis drives the evolution of gene amplification in cancer. Nature. 2020. doi:10.1038/s41586-020-03064-z

12. Koche RP, Rodriguez-Fos E, Helmsauer K, et al. Extrachromosomal circular DNA drives oncogenic genome remodeling in neuroblastoma. Nat Genet. 2020;52(1):29-34. doi:10.1038/s41588-019-0547-z

13. Kim H, Nguyen N-P, Turner K, et al. Extrachromosomal DNA is associated with oncogene amplification and poor outcome across multiple cancers. Nat Genet. 2020;52(9):891-897. doi:10.1038/s41588-0200678-2

1112 14. Turner KM, Deshpande V, Beyter D, et al. Extrachromosomal oncogene amplification drives tumour evolution and genetic heterogeneity. Nature. 2017;543(7643):122-125. doi:10.1038/nature21356

1114 15. Xu K, Ding L, Chang T-C, et al. Structure and evolution of double minutes in diagnosis and relapse brain tumors. Acta Neuropathol. 2019;137(1):123-137. doi:10.1007/s00401-018-1912-1

16. Northcott PA, Lee C, Zichner T, et al. Enhancer hijacking activates GFI1 family oncogenes in medulloblastoma. Nature. 2014;511(7510):428-434. doi:10.1038/nature13379

1118 17. Bandopadhayay $P$, Ramkissoon LA, Jain P, et al. MYB-QKI rearrangements in angiocentric glioma drive tumorigenicity through a tripartite mechanism. Nat Genet. 2016;48:273. http://dx.doi.org/10.1038/ng.3500.

18. Beroukhim R, Zhang X, Meyerson M. Copy number alterations unmasked as enhancer hijackers. Nat Genet. 2017;49(1):5-6. doi:10.1038/ng.3754

19. Chen CCL, Deshmukh S, Jessa S, et al. Histone H3.3G34-Mutant Interneuron Progenitors Co-opt PDGFRA for Gliomagenesis. Cell. 2020. doi:https://doi.org/10.1016/j.cell.2020.11.012

20. Helmsauer K, Valieva ME, Ali S, et al. Enhancer hijacking determines extrachromosomal circular MYCN 

19452-y

1128 21. Morton AR, Dogan-Artun N, Faber ZJ, et al. Functional Enhancers Shape Extrachromosomal Oncogene Amplifications. Cell. 2019;179(6):1330-1341.e13. doi:https://doi.org/10.1016/j.cell.2019.10.039

1130 22. Rheinbay E, Nielsen MM, Abascal F, et al. Analyses of non-coding somatic drivers in 2,658 cancer whole genomes. Nature. 2020. doi:10.1038/s41586-020-1965-x

1132 23. Li Y, Roberts ND, Wala JA, et al. Patterns of somatic structural variation in human cancer genomes. Nature. 2020;578(7793):112-121. doi:10.1038/s41586-019-1913-9

1134 24. Kim J, Mouw KW, Polak P, et al. Somatic ERCC2 mutations are associated with a distinct genomic signature in urothelial tumors. Nat Genet. 2016;48:600. http://dx.doi.org/10.1038/ng.3557.

1136 25. Alexandrov LB, Kim J, Haradhvala NJ, et al. The repertoire of mutational signatures in human cancer. Nature. 2020;578(7793):94-101. doi:10.1038/s41586-020-1943-3

1138 26. Nik-Zainal S, Davies H, Staaf J, et al. Landscape of somatic mutations in 560 breast cancer whole-genome sequences. Nature. 2016;534:47. https://doi.org/10.1038/nature17676.

27. Hillman RT, Chisholm GB, Lu KH, Futreal PA. Genomic Rearrangement Signatures and Clinical Outcomes in High-Grade Serous Ovarian Cancer. JNCI J Natl Cancer Inst. 2018;110(3):265-272. doi:10.1093/jnci/djx176

1142 28. Zhang AW, McPherson A, Milne K, et al. Interfaces of Malignant and Immunologic Clonal Dynamics in Ovarian Cancer. Cell. 2018;173(7):1755-1769.e22. doi:https://doi.org/10.1016/j.cell.2018.03.073

1144 29. Angus L, Smid M, Wilting SM, et al. The genomic landscape of metastatic breast cancer highlights changes in mutation and signature frequencies. Nat Genet. 2019;51(10):1450-1458. doi:10.1038/s41588-0190507-7

30. Morganella S, Alexandrov LB, Glodzik D, et al. The topography of mutational processes in breast cancer genomes. Nat Commun. 2016;7(1):11383. doi:10.1038/ncomms11383

31. Bayard Q, Meunier L, Peneau C, et al. Cyclin A2/E1 activation defines a hepatocellular carcinoma subclass with a rearrangement signature of replication stress. Nat Commun. 2018;9(1):5235. doi:10.1038/s41467018-07552-9

1152 32. Puget S, Beccaria K, Blauwblomme T, et al. Biopsy in a series of 130 pediatric diffuse intrinsic Pontine gliomas. Child's Nerv Syst. 2015;31(10):1773-1780. doi:10.1007/s00381-015-2832-1

1154 33. Roujeau T, Machado G, Garnett MR, et al. Stereotactic biopsy of diffuse pontine lesions in children. J Neurosurg Pediatr PED. 107(1):1-4. doi:10.3171/PED-07/07/001

1156 34. Cage TA, Samagh SP, Mueller S, et al. Feasibility, safety, and indications for surgical biopsy of intrinsic brainstem tumors in children. Child's Nerv Syst. 2013;29(8):1313-1319. doi:10.1007/s00381-013-2101-0

1158 35. Gupta N, Goumnerova LC, Manley P, et al. Prospective feasibility and safety assessment of surgical biopsy for patients with newly diagnosed diffuse intrinsic pontine glioma. Neuro Oncol. 2018;20(11):1547-1555. doi:10.1093/neuonc/noy070

36. Wala JA, Bandopadhayay P, Greenwald NF, et al. SvABA: genome-wide detection of structural variants and indels by local assembly. Genome Res . 2018;28(4):581-591. doi:10.1101/gr.221028.117

37. Wala JA, Shapira O, Li Y, et al. Selective and mechanistic sources of recurrent rearrangements across the cancer genome. bioRxiv. January 2017. http://biorxiv.org/content/early/2017/09/14/187609.abstract.

38. Bahr C, von Paleske L, Uslu V V, et al. A Myc enhancer cluster regulates normal and leukaemic haematopoietic stem cell hierarchies. Nature. 2018;553:515. https://doi.org/10.1038/nature25193.

39. Fulco CP, Munschauer M, Anyoha R, et al. Systematic mapping of functional enhancer-promoter connections with CRISPR interference. Science (80- ). 2016;354(6313):769 LP - 773. doi:10.1126/science.aag2445

1170 40. Zimmerman MW (Partners HD a S of H-RPN and IA through MIEH and FEA pd., Liu Y, He S, et al. $<$ em $>$ MYC $</$ em $>$ Drives a Subset of High-Risk Pediatric Neuroblastomas and Is Activated through Mechanisms Including Enhancer Hijacking and Focal Enhancer Amplification. Cancer Discov. 2018;8(3):320 LP - 335. doi:10.1158/2159-8290.CD-17-0993

1174 41. Zhang X, Choi PS, Francis JM, et al. Identification of focally amplified lineage-specific super-enhancers in human epithelial cancers. Nat Genet. 2015;48(2):176-182. doi:10.1038/ng.3470

1176 42. Krug B, De Jay N, Harutyunyan AS, et al. Pervasive H3K27 Acetylation Leads to ERV Expression and a Therapeutic Vulnerability in H3K27M Gliomas. Cancer Cell. 2019;35(5):782-797.e8. 
43. Suvà ML, Rheinbay E, Gillespie SM, et al. Reconstructing and Reprogramming the Tumor-Propagating Potential of Glioblastoma Stem-like Cells. Cell. 2014;157(3):580-594.

doi:https://doi.org/10.1016/j.cell.2014.02.030

44. Nagaraja S, Quezada MA, Gillespie SM, et al. Histone Variant and Cell Context Determine H3K27M Reprogramming of the Enhancer Landscape and Oncogenic State. Mol Cell. 2019;76(6):965-980.e12. doi:https://doi.org/10.1016/j.molcel.2019.08.030

45. Dunham I, Kundaje A, Aldred SF, et al. An integrated encyclopedia of DNA elements in the human genome. Nature. 2012;489(7414):57-74. doi:10.1038/nature11247

46. Davis CA, Hitz BC, Sloan CA, et al. The Encyclopedia of DNA elements (ENCODE): data portal update. Nucleic Acids Res. 2017;46(D1):D794-D801. doi:10.1093/nar/gkx1081

47. Lee SB, Frattini V, Bansal M, et al. An ID2-dependent mechanism for VHL inactivation in cancer. Nature. 2016;529(7585):172-177. doi:10.1038/nature16475

48. Paolella BR, Havrda MC, Mantani A, Wray CM, Zhang Z, Israel MA. p53 Directly Represses Id2 to Inhibit the Proliferation of Neural Progenitor Cells. Stem Cells. 2011;29(7):1090-1101. doi:10.1002/stem.660

49. Frankell AM, Jammula S, Li X, et al. The landscape of selection in 551 esophageal adenocarcinomas defines genomic biomarkers for the clinic. Nat Genet. 2019;51(3):506-516. doi:10.1038/s41588-0180331-5

1196 50. Verhaak RGW, Bafna V, Mischel PS. Extrachromosomal oncogene amplification in tumour pathogenesis and evolution. Nat Rev Cancer. 2019;19(5):283-288. doi:10.1038/s41568-019-0128-6

1198 51. Pathania M, De Jay N, Maestro N, et al. H3.3 ${ }^{\mathrm{K} 27 \mathrm{M}}$ Cooperates with <em $>\operatorname{Trp53}</$ em $>$ Loss and PDGFRA Gain in Mouse Embryonic Neural Progenitor Cells to Induce Invasive High-Grade Gliomas. Cancer Cell. 2017;32(5):684-700.e9. doi:10.1016/j.ccell.2017.09.014

52. Flavahan WA, Drier Y, Liau BB, et al. Insulator dysfunction and oncogene activation in IDH mutant

53. Sondka Z, Bamford S, Cole CG, Ward SA, Dunham I, Forbes SA. The COSMIC Cancer Gene Census: gliomas. Nature. 2016;529(7584):110-114. doi:10.1038/nature16490

describing genetic dysfunction across all human cancers. Nat Rev Cancer. 2018;18(11):696-705. doi:10.1038/s41568-018-0060-1

54. Menghi F, Barthel FP, Yadav V, et al. The Tandem Duplicator Phenotype Is a Prevalent Genome-Wide Cancer Configuration Driven by Distinct Gene Mutations. Cancer Cell. 2018;34(2):197-210.e5.

55. Hoopes JI, Cortez LM, Mertz TM, Malc EP, Mieczkowski PA, Roberts SA. APOBEC3A and APOBEC3B doi:https://doi.org/10.1016/j.ccell.2018.06.008 Preferentially Deaminate the Lagging Strand Template during DNA Replication. Cell Rep. 2016;14(6):1273-1282. doi:10.1016/j.celrep.2016.01.021

56. Haradhvala NJ, Polak P, Stojanov P, et al. Mutational Strand Asymmetries in Cancer Genomes Reveal Mechanisms of DNA Damage and Repair. Cell. 2016;164(3):538-549. doi:https://doi.org/10.1016/j.cell.2015.12.050 Characteristics of Long-Term Survivors of Diffuse Intrinsic Pontine Glioma (DIPG): A Collaborative Report From the International and European Society for Pediatric Oncology DIPG Registries. J Clin Oncol. 2018;36(19):1963-1972. doi:10.1200/JCO.2017.75.9308 Genet. 2013;45:1134. http://dx.doi.org/10.1038/ng.2760.

59. Taylor AM, Shih J, Ha G, et al. Genomic and Functional Approaches to Understanding Cancer Aneuploidy.

60. LETT JT, CALDWELLI, DEAN CJ, ALEXANDER P. Rejoining of X-ray Induced Breaks in the DNA of Leukaemia Cells. Nature. 1967;214(5090):790-792. doi:10.1038/214790a0

61. Gerstung M, Jolly C, Leshchiner I, et al. The evolutionary history of 2,658 cancers. Nature. 2020;578(7793):122-128. doi:10.1038/s41586-019-1907-7

62. Larson JD, Kasper LH, Paugh BS, et al. Histone H3.3 K27M Accelerates Spontaneous Brainstem Glioma and Drives Restricted Changes in Bivalent Gene Expression. Cancer Cell. 2019;35(1):140-155.e7. doi:https://doi.org/10.1016/j.ccell.2018.11.015 
63. Cordero FJ, Huang Z, Grenier C, et al. Histone H3.3K27M Represses \&lt;em\&gt;p16\&lt;/em\&gt; to Accelerate Gliomagenesis in a Murine Model of DIPG. Mol Cancer Res. 2017;15(9):1243 LP - 1254.

1232

1234

1236

1238

1240

1242

1244

1246

1248

1250

1252

1254

1256

1258

1260

1262

1264

1266

1268

1270

1272

1274

1276

1278

1280 doi:10.1158/1541-7786.MCR-16-0389

64. Funato K, Major T, Lewis PW, Allis CD, Tabar V. Use of human embryonic stem cells to model pediatric gliomas with H3.3K27M histone mutation. Science (80- ). 2014;346(6216):1529 LP - 1533. doi:10.1126/science.1253799

65. Hoffman LM, DeWire M, Ryall S, et al. Spatial genomic heterogeneity in diffuse intrinsic pontine and midline high-grade glioma: implications for diagnostic biopsy and targeted therapeutics. Acta Neuropathol Commun. 2016;4(1):1. doi:10.1186/s40478-015-0269-0

66. Nikbakht H, Panditharatna E, Mikael LG, et al. Spatial and temporal homogeneity of driver mutations in diffuse intrinsic pontine glioma. Nat Commun. 2016;7(1):11185. doi:10.1038/ncomms11185

67. Salloum R, McConechy MK, Mikael LG, et al. Characterizing temporal genomic heterogeneity in pediatric high-grade gliomas. Acta Neuropathol Commun. 2017;5(1):78. doi:10.1186/s40478-017-0479-8

68. Vinci M, Burford A, Molinari V, et al. Functional diversity and cooperativity between subclonal populations of pediatric glioblastoma and diffuse intrinsic pontine glioma cells. Nat Med. 2018;24(8):1204-1215. doi:10.1038/s41591-018-0086-7

69. Castel D, Philippe C, Calmon R, et al. Histone H3F3A and HIST1H3B K27M mutations define two subgroups of diffuse intrinsic pontine gliomas with different prognosis and phenotypes. Acta Neuropathol. 2015;130(6):815-827. doi:10.1007/s00401-015-1478-0

70. Khuong-Quang D-A, Buczkowicz P, Rakopoulos P, et al. K27M mutation in histone H3.3 defines clinically and biologically distinct subgroups of pediatric diffuse intrinsic pontine gliomas. Acta Neuropathol. 2012;124(3):439-447. doi:10.1007/s00401-012-0998-0

71. Beroukhim R, Mermel C, Porter D, et al. The landscape of somatic copy-number alteration across human cancers. Nature. 2010;463. doi:10.1038/nature08822

72. Wu S, Turner KM, Nguyen N, et al. Circular ecDNA promotes accessible chromatin and high oncogene expression. Nature. 2019;575(7784):699-703. doi:10.1038/s41586-019-1763-5

73. Nathanson DA, Gini B, Mottahedeh J, et al. Targeted Therapy Resistance Mediated by Dynamic Regulation of Extrachromosomal Mutant EGFR DNA. Science (80- ). 2014;343(6166):72 LP - 76. doi:10.1126/science.1241328

74. Miklja Z, Yadav VN, Cartaxo RT, et al. Everolimus improves the efficacy of dasatinib in PDGFR $\alpha$-driven glioma. J Clin Invest. 2020;130(10):5313-5325. doi:10.1172/JCI133310

75. Miklja Z, Pasternak A, Stallard S, et al. Molecular profiling and targeted therapy in pediatric gliomas: review and consensus recommendations. Neuro Oncol. 2019;21(8):968-980. doi:10.1093/neuonc/noz022

76. Gröbner SN, Worst BC, Weischenfeldt J, et al. The landscape of genomic alterations across childhood cancers. Nature. 2018;555(7696):321-327. doi:10.1038/nature25480

77. Zhang Y, Chen F, Donehower LA, Scheurer ME, Creighton CJ. A pediatric brain tumor atlas of genes deregulated by somatic genomic rearrangement. Nat Commun. 2021;12(1):937. doi:10.1038/s41467021-21081-y

78. Rausch T, Jones DTW, Zapatka M, et al. Genome Sequencing of Pediatric Medulloblastoma Links Catastrophic DNA Rearrangements with TP53 Mutations. Cell. 2012;148(1):59-71. doi:https://doi.org/10.1016/j.cell.2011.12.013

79. Li H, Durbin R. Fast and accurate short read alignment with Burrows-Wheeler transform. Bioinformatics. 2009;25(14):1754-1760. http://dx.doi.org/10.1093/bioinformatics/btp324.

80. Benjamin D, Sato T, Cibulskis K, Getz G, Stewart C, Lichtenstein L. Calling Somatic SNVs and Indels with Mutect2. bioRxiv. January 2019:861054. doi:10.1101/861054

81. Lawrence MS, Stojanov P, Polak P, et al. Mutational heterogeneity in cancer and the search for new cancer-associated genes. Nature. 2013;499:214. https://doi.org/10.1038/nature12213.

82. Tabak B, Saksena G, Oh C, et al. The Tangent copy-number inference pipeline for cancer genome analyses. bioRxiv. January 2019:566505. doi:10.1101/566505

83. Carter SL, Cibulskis K, Helman E, et al. Absolute quantification of somatic DNA alterations in human cancer. Nat Biotechnol. 2012;30:413. https://doi.org/10.1038/nbt.2203.

84. Viswanathan SR, Ha G, Hoff AM, et al. Structural Alterations Driving Castration-Resistant Prostate Cancer 
Revealed by Linked-Read Genome Sequencing. Cell. 2018;174(2):433-447.e19. doi:https://doi.org/10.1016/j.cell.2018.05.036

1284

1286

1288

1290

1292

1294

1296

1298

1300

1302

1304

1306

1308

1310

1312

1314

1316

1318

1320

1322

1324

1326

1328

1330

1332

85. Mermel CH, Schumacher SE, Hill B, Meyerson ML, Beroukhim R, Getz G. GISTIC2.0 facilitates sensitive and confident localization of the targets of focal somatic copy-number alteration in human cancers. Genome Biol. 2011;12(4):R41. doi:10.1186/gb-2011-12-4-r41

86. Won $\mathrm{H}$, de la Torre-Ubieta L, Stein JL, et al. Chromosome conformation elucidates regulatory relationships in developing human brain. Nature. 2016;538(7626):523-527. doi:10.1038/nature19847

87. Li H. Toward better understanding of artifacts in variant calling from high-coverage samples. Bioinformatics. 2014;30(20):2843-2851. doi:10.1093/bioinformatics/btu356

88. Imielinski M, Guo G, Meyerson M. Insertions and Deletions Target Lineage-Defining Genes in Human Cancers. Cell. 2017;168(3):460-472.e14. doi:https://doi.org/10.1016/j.cell.2016.12.025

89. Smith DI, Zhu Y, McAvoy S, Kuhn R. Common fragile sites, extremely large genes, neural development and cancer. Cancer Lett. 2006;232(1):48-57. doi:https://doi.org/10.1016/j.canlet.2005.06.049

90. Hadi K, Yao X, Behr JM, et al. Distinct Classes of Complex Structural Variation Uncovered across Thousands of Cancer Genome Graphs. Cell. 2020;183(1):197-210.e32. doi:10.1016/j.cell.2020.08.006

91. Benjamini Y, Hochberg Y. Controlling the false discovery rate: a practical and powerful approach to multiple testing. J R Stat Soc B. 1995;57.

92. Dobin A, Davis CA, Schlesinger F, et al. STAR: ultrafast universal RNA-seq aligner. Bioinformatics. 2012;29(1):15-21. doi:10.1093/bioinformatics/bts635

93. DeLuca DS, Levin JZ, Sivachenko A, et al. RNA-SeQC: RNA-seq metrics for quality control and process optimization. Bioinformatics. 2012;28(11):1530-1532. doi:10.1093/bioinformatics/bts196

94. Aguet F, Brown AA, Castel SE, et al. Genetic effects on gene expression across human tissues. Nature. 2017;550(7675):204-213. doi:10.1038/nature24277

95. Love MI, Huber W, Anders S. Moderated estimation of fold change and dispersion for RNA-seq data with

96. Johnson WE, Li C, Rabinovic A. Adjusting batch effects in microarray expression data using empirical Bayes methods. Biostatistics. 2006;8(1):118-127. doi:10.1093/biostatistics/kxj037

97. Leek JT, Johnson WE, Parker HS, Jaffe AE, Storey JD. The sva package for removing batch effects and other unwanted variation in high-throughput experiments. Bioinformatics. 2012;28(6):882-883.

doi:10.1093/bioinformatics/bts034

98. Zhang Y, Liu T, Meyer CA, et al. Model-based Analysis of ChIP-Seq (MACS). Genome Biol. 2008;9(9):R137. doi:10.1186/gb-2008-9-9-r137

99. Rao SSP, Huntley MH, Durand NC, et al. A 3D Map of the Human Genome at Kilobase Resolution Reveals Principles of Chromatin Looping. Cell. 2014;159(7):1665-1680. doi:10.1016/j.cell.2014.11.021

100. Rajarajan $\mathrm{P}$, Borrman $\mathrm{T}$, Liao W, et al. Neuron-specific signatures in the chromosomal connectome associated with schizophrenia risk. Science (80- ). 2018;362(6420). doi:10.1126/science.aat4311

101. Durand NC, Robinson JT, Shamim MS, et al. Juicebox Provides a Visualization System for Hi-C Contact Maps with Unlimited Zoom. Cell Syst. 2016;3(1):99-101. doi:10.1016/j.cels.2015.07.012

102. Cameron CJF, Dostie J, Blanchette M. HIFI: estimating DNA-DNA interaction frequency from Hi-C data at restriction-fragment resolution. Genome Biol. 2020;21(1):11. doi:10.1186/s13059-019-1913-y

103. Grasso CS, Tang Y, Truffaux N, et al. Functionally defined therapeutic targets in diffuse intrinsic pontine glioma. Nat Med. 2015;21(6):555-559. doi:10.1038/nm.3855

104. Wilkerson MD, Hayes DN. ConsensusClusterPlus: a class discovery tool with confidence assessments and item tracking. Bioinformatics. 2010;26(12):1572-1573. doi:10.1093/bioinformatics/btq170

105. Ramos AH, Lichtenstein L, Gupta M, et al. Oncotator: Cancer Variant Annotation Tool. Hum Mutat. 2015;36(4):E2423-E2429. doi:10.1002/humu.22771

106. Cerami E, Gao J, Dogrusoz U, et al. The cBio Cancer Genomics Portal: An Open Platform for Exploring Multidimensional Cancer Genomics Data. Cancer Discov. 2012;2(5):401 LP - 404. doi:10.1158/21598290.CD-12-0095

107. Gao J, Aksoy BA, Dogrusoz U, et al. Integrative Analysis of Complex Cancer Genomics and Clinical Profiles Using the cBioPortal. Sci Signal. 2013;6(269):pl1 LP-pl1. doi:10.1126/scisignal.2004088

108. Amin SB, Anderson KJ, Boudreau CE, et al. Comparative Molecular Life History of Spontaneous Canine 
1334 and Human Gliomas. Cancer Cell. 2020;37(2):243-257.e7. doi:https://doi.org/10.1016/j.ccell.2020.01.004

109. Shinde J, Bayard Q, Imbeaud S, et al. Palimpsest: an R package for studying mutational and structural 1336 variant signatures along clonal evolution in cancer. Bioinformatics. 2018;34(19):3380-3381. doi:10.1093/bioinformatics/bty388

1338 110. Caravagna G, Heide T, Williams MJ, et al. Subclonal reconstruction of tumors by using machine learning and population genetics. Nat Genet. 2020;52(9):898-907. doi:10.1038/s41588-020-0675-5

1340 111. Storey JD. False Discovery Rate BT - International Encyclopedia of Statistical Science. In: Lovric M, ed. Berlin, Heidelberg: Springer Berlin Heidelberg; 2011:504-508. doi:10.1007/978-3-642-04898-2_248 
A Significantly Recurrent Breakpoints

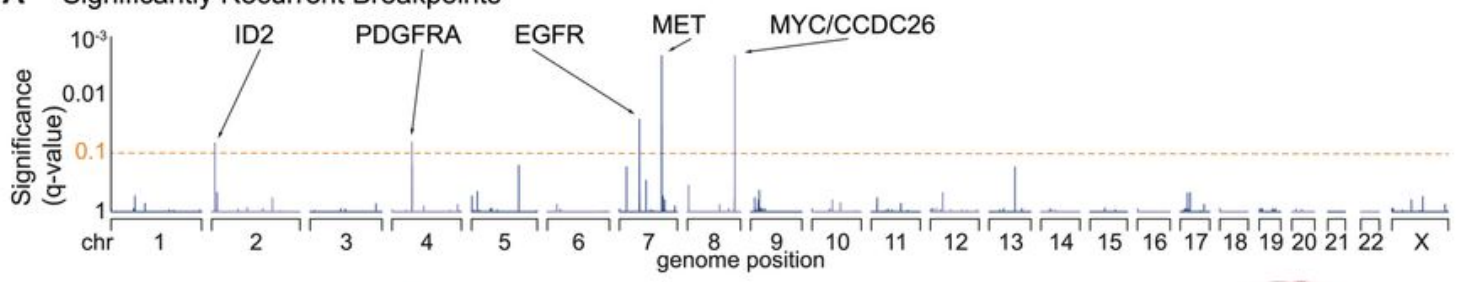

B

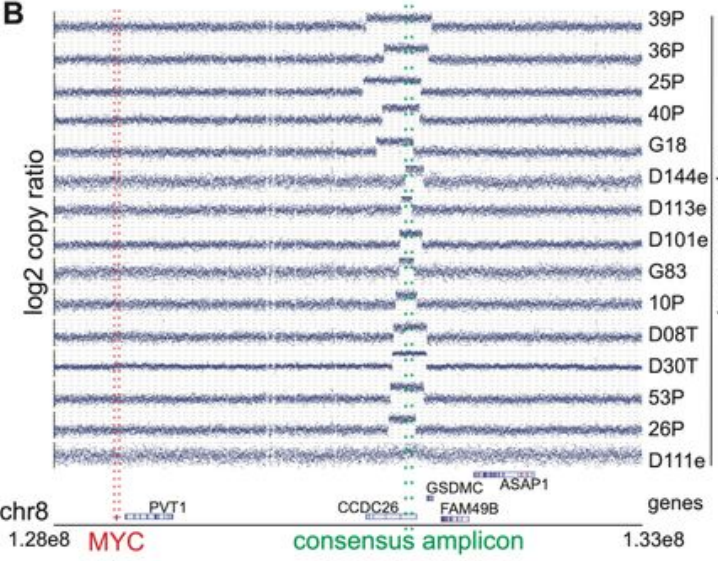

D
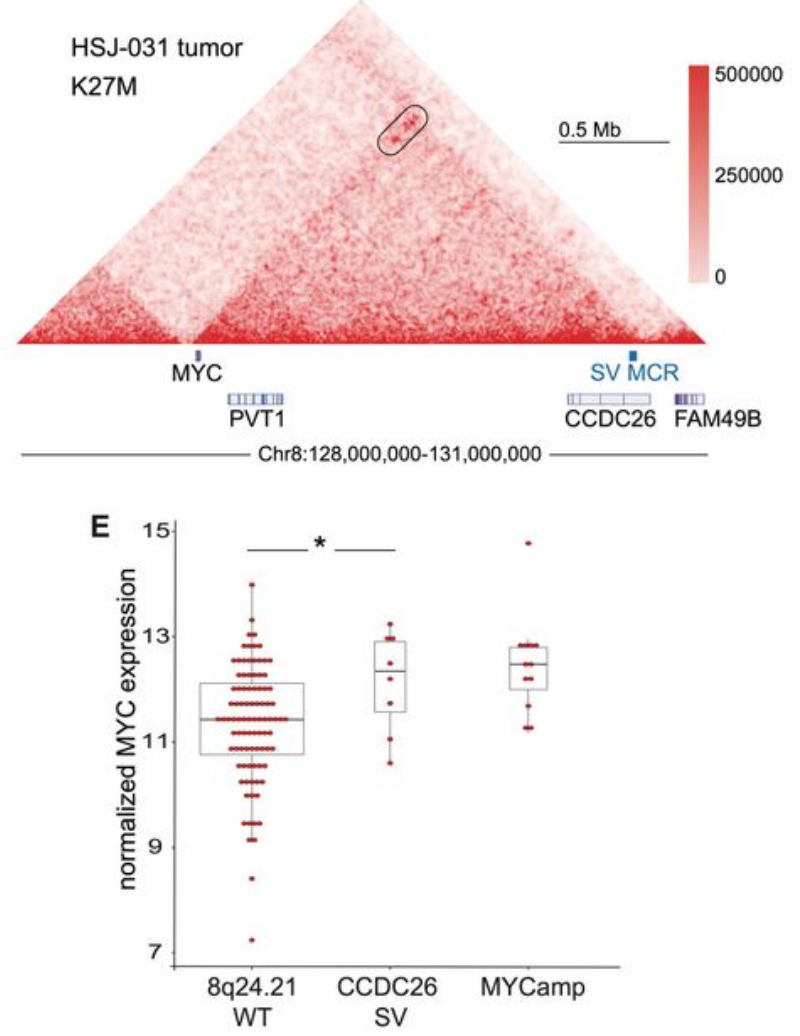

C recurring SVs , men en

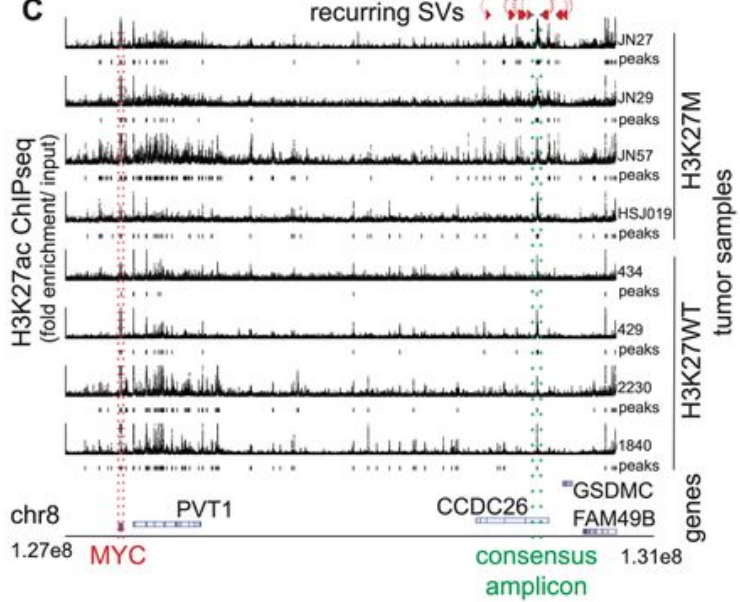

$\mathbf{F}$

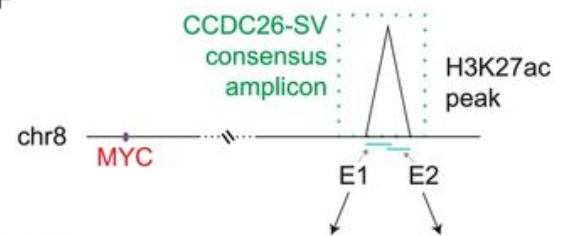

G

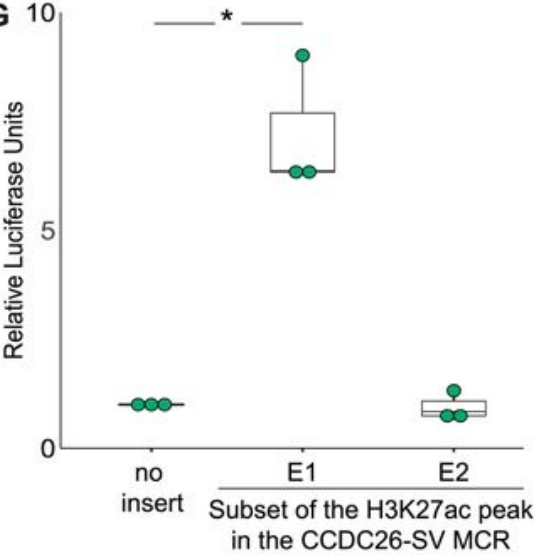

\section{Figure 1}

Significantly recurrent breakpoints within CCDC26. (A) Significance (multiple hypothesis corrected qvalues, vertical axis) of recurrent breakpoints (genomic positions on the horizontal axis) across the 179 pHGG genomes. (B) Copy-number profiles across the MYC TAD for the 15 tumors with the recurrent 
CCDC26 SV. (C) H3K27ac ChIP seq tracks within the TAD containing MYC (red lines) and CCDC26, for four H3K27M and four H3K27WT pHGGs tumors. Significantly enriched peaks (q-value < 0.01 ) are indicated below each H3K27ac ChIP-seq track. The CCDC26 amplicon boundaries for individual samples are indicated by the paired red arrows at the top. The consensus amplicon is indicated by the green dotted lines, and centers on an H3K27ac peak. (D) Hi-C heatmap across the MYC-CCDC26 locus from a midline glioma. Increasing interaction frequencies are indicated by brighter shades of red. The black oval outlines the frequent connections between MYC and the H3K27ac-enriched region within CCDC26. The minimal common region of amplification (SV MCR) is indicated at the bottom. (E) Normalized MYC expression in DMG samples with wild-type copy-number profiles at 8q24.21 $(n=92)$, CCDC26-SVs $(n=8)$ or amplifications of the MYC coding sequence $(n=12)$. *denotes a $p<0.05$ as determined by Wilcoxon rank sum test. (F) Schematic illustrating the luciferase reporter used to validate the enhancer in CCDC26, showing the positions of the E1 and E2 sequences with respect to the enhancer within CCDC26. (G) Normalized luciferase activity in DIPG13 cells following transduction of the E1 and E2 enhancer reporters or empty vector controls. Values represent the average of four technical replicates in each of three independent experiments. * denotes $p=0.019$, T-test (unpaired, two-sided). 
A

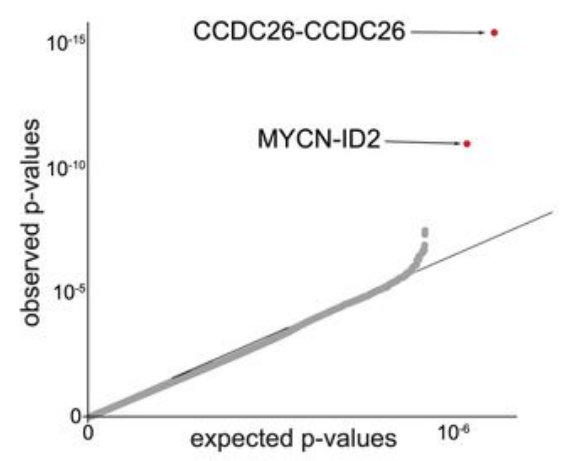

C

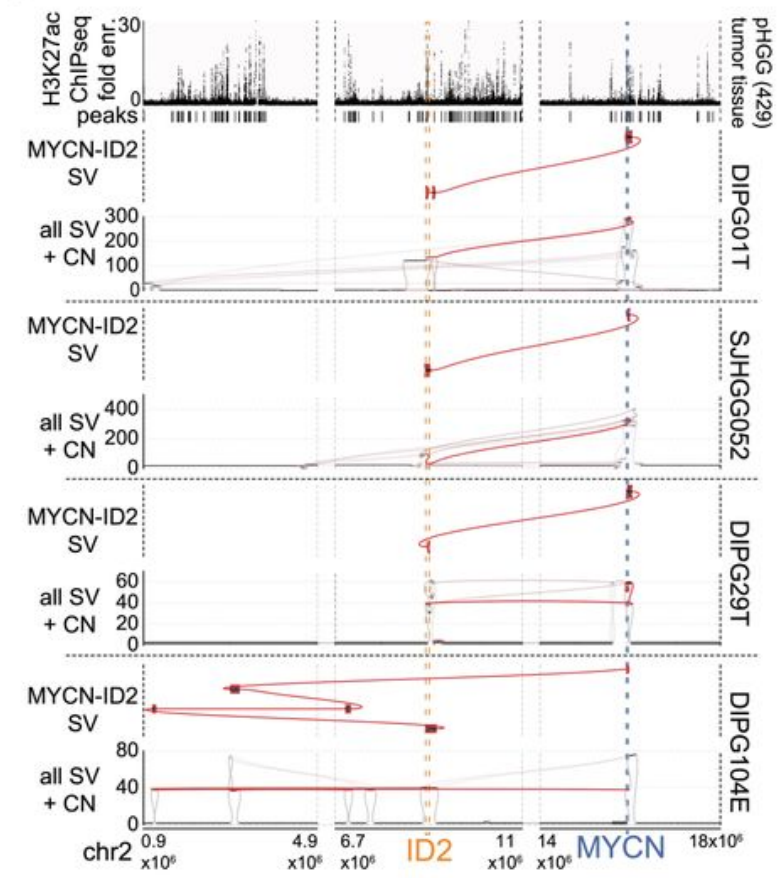

$\mathbf{F}$

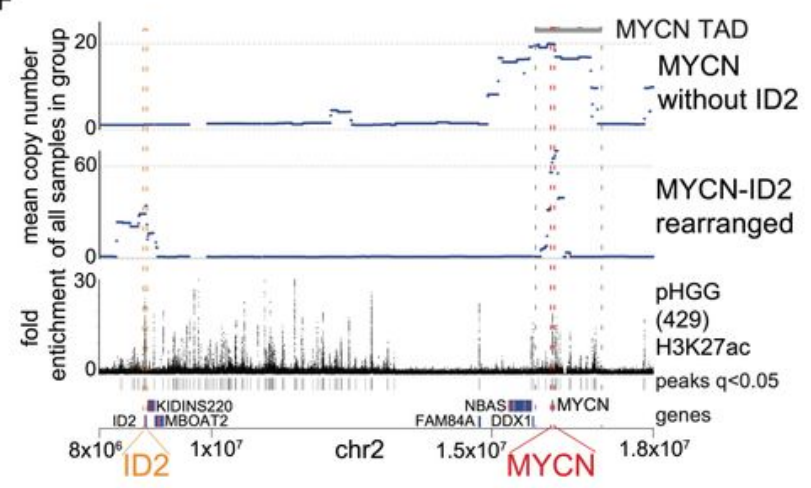

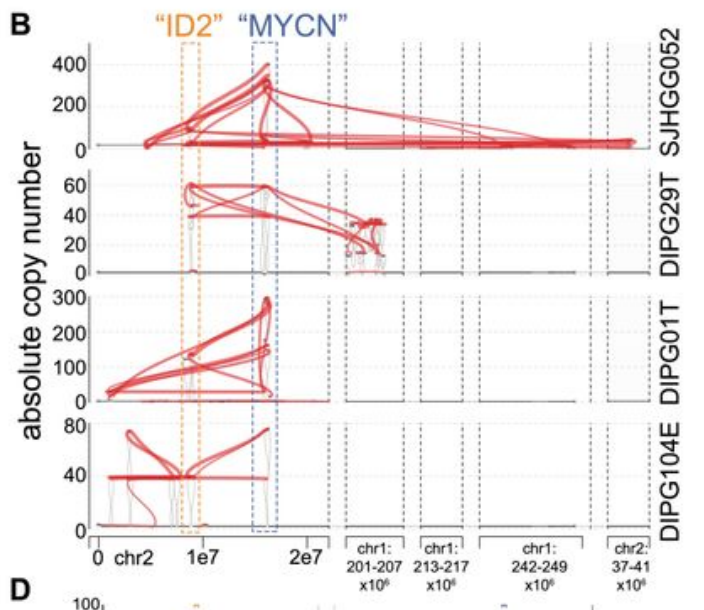
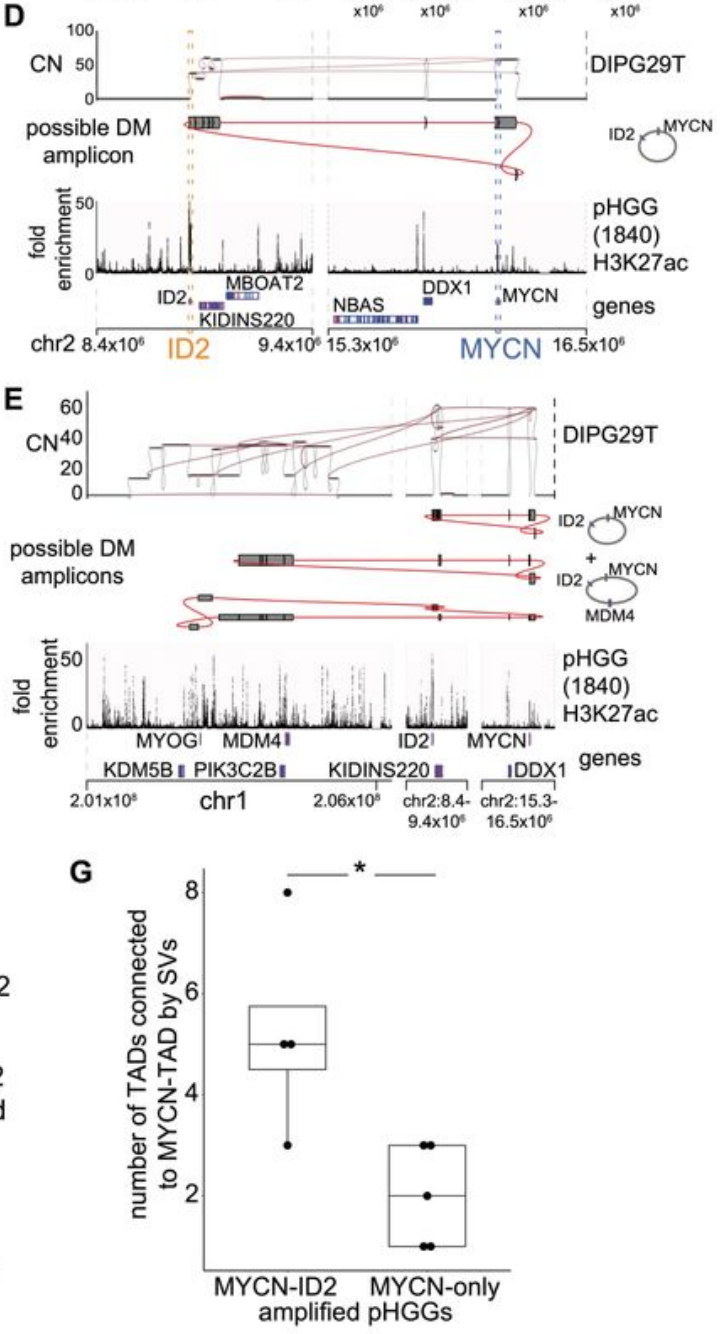

\section{Figure 2}

Significantly recurrent juxtapositions: MYCN-ID2. (A) Quantile-Quantile plot indicating the significance of juxtapositions between pairs of genomic loci. SVs that reached statistical significance are depicted in red. (B) SVs (red lines) involving MYCN and ID2 in samples with MYCN-ID2 rearrangements, and the number of copies at each connected locus (vertical axis). The dashed boxes indicate loci encompassing ID2 and MYCN. (C) SV maps as in panel B, with SVs juxtaposing ID2 and MYCN highlighted in red. The top track 
indicates H3K27ac marks in a pHGG without a known MYCN-ID2 rearrangement, showing a strong enhancer within ID2. In each case, the MYCN-ID2 juxtaposition reduces the somatic distance MYCN and ID2 (each indicated by a dashed line) from $7 \mathrm{Mbp}$ to less than 700kbp. (D-E) Examples of possible reconstructions of circular extrachromosomal amplicons containing MYCN and ID2 from a single DMG. (D) The simplest possible circle connecting MYCN and ID2. The top track shows copy-number and SVs, the middle track indicates the reconstructed topology, and the bottom track shows H3K27ac binding at the indicated loci in a different PHGG. (E) The chr1 loci connected to the MYCN-ID complex in this DMG. Short-read reconstructions allow for several extrachromosomal amplicons incorporating MYCN-ID2 and MDM4. The difference in the copy number could be explained either by a mix of amplicons containing respectively MYCN-ID2 alone and MYCN-ID2-MDM4 or by more complex amplicons incorporating multiple copies of MYCN878 ID2 for each copy of MDM4. (E) MYCN amplicons in tumors without ID2 amplification (top track) incorporate a larger fraction of the MYCN TAD $(60 \%)$ relative to MYCN amplicons in tumors with MYCN-ID2-SV (second track; 23\%, $p=0.03$, T-test). As a result, the former tend to include more loci with H3K27ac enrichment (third track) from the MYCN-TAD; significantly enriched H3K27ac peaks (q-value < 0.01) are indicated with black bars between the H3K27ac fold enrichment and the gene track. (F) MYCN-ID2 amplified pHGGs contain significantly more SVs between the MYCN-TAD and other TADs than pHGGs amplifying only MYCN and its neighborhood. ( $p=0.03$, Wilcoxon rank sum test). 

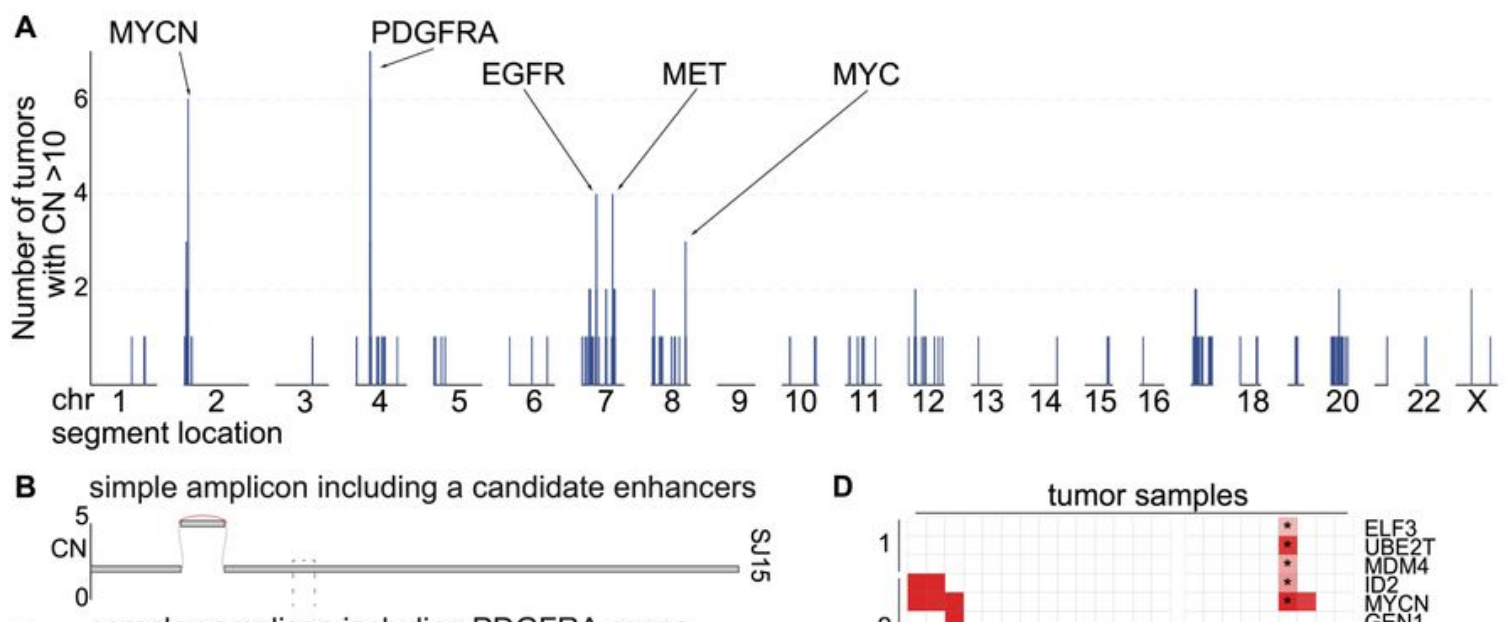

C complex amplicon including PDGFRA exons

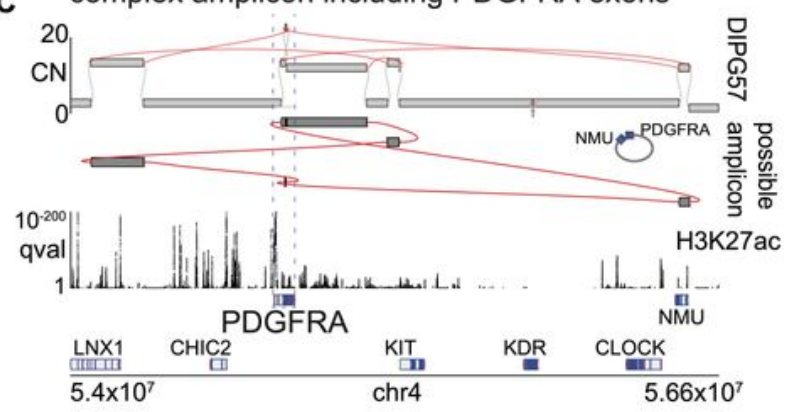

E complex amplicon of EGFR and CCNE1
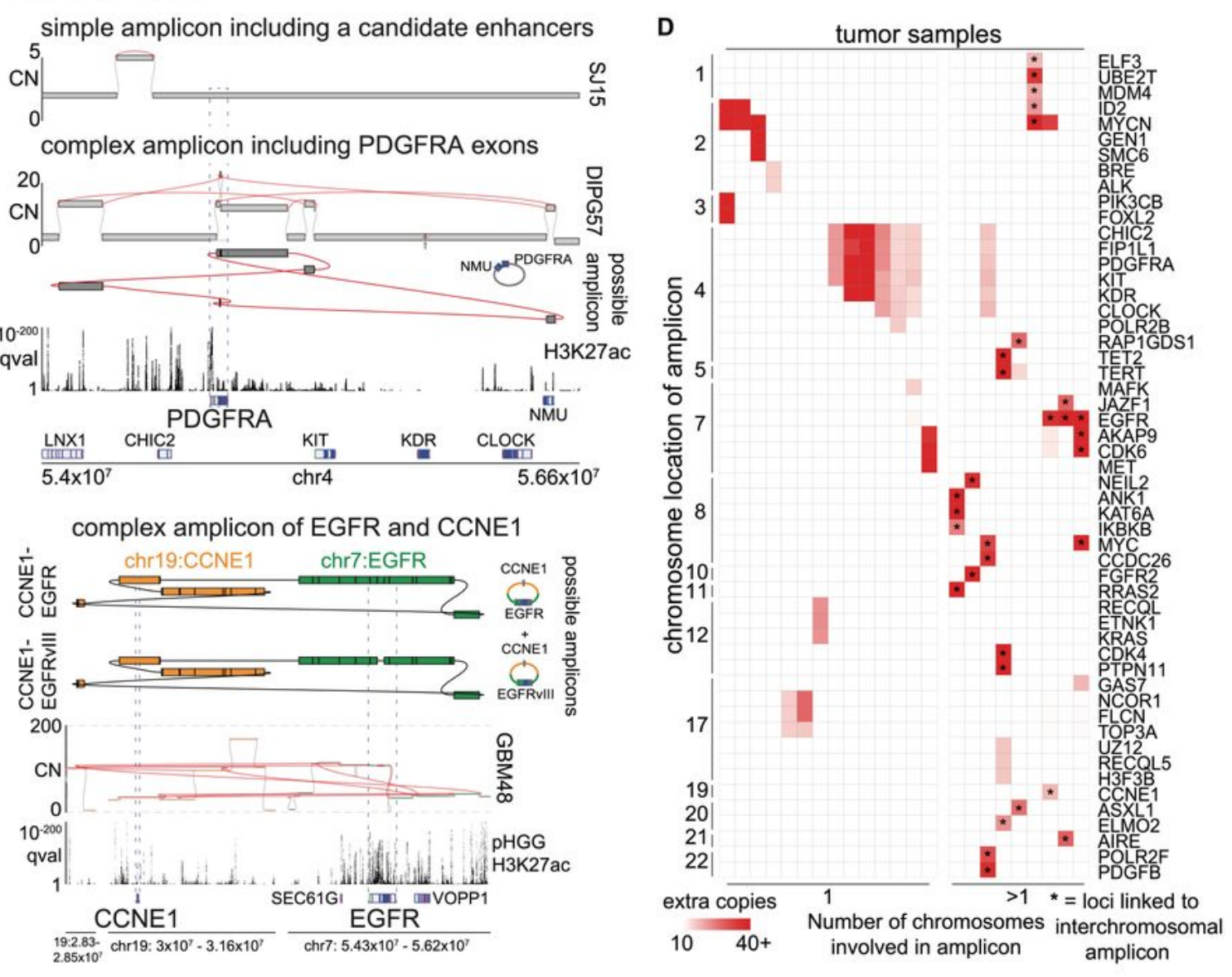

\section{Figure 3}

High-level amplicons. (A) For each genomic locus (horizontal axis), the number of tumors containing a high-level $(\mathrm{CN}>10)$ amplicon is indicated (vertical axis). SRBs are highlighted the top. (B-C) Simple and complex-SVs exhibit distinct mechanisms to active PDGFRA. The top and bottom tracks indicate copynumbers and the significance of H3K27ac enrichment (as calculated from eight pHGGs), respectively. SVs are highlighted in red. Selected gene loci are indicated on the bottom. (B) A simple amplicon of a 
region with known19,52 PDGFRA enhancers. (C) A complex high-level PDGFRA amplicon, displayed as in panel (B) with the addition of a track (second from top) indicating the topology of the amplicon. The complex-SV cluster around PDGFRA connects several segments on chr4, which are amplified to ten absolute copies. The SV calls support the reconstruction of an extrachromosomal amplicon incorporating PDGFRA exons and these segments. (D) Cancer genes involved in high-level amplicons ( $>10$ copies) within the cohort. 9/23 tumors (grouped on right) contain high-level amplicons encompassing 898 loci from two or more chromosomes. These linked loci are marked by *. The color of each cell represents the number of extra copies due to the amplicon. (E) Example of a tumor with an extrachromosomal amplicon including two oncogenes from different chromosomes. This tumor shows a cluster of SVs connecting the EGFR and CCNE1 loci. The regions of both oncogenes are amplified to different CNs but in both cases reach several dozen absolute copies. (top) The complexity of the SVs allows for the reconstruction of several possible extrachromosomal amplicons. The $\mathrm{CN}$ differences in the bulk profile (middle) could be explained by either a mix of different circles or by more complex circles incorporating some segments repeatedly. The SV calls also reveal that a small fraction of the EGFR amplicons in this patient already show the EGFRvIll variant. The bottom two tracks show the genes of interest at the location and q-value H3K27ac track calculated from eight pHGG tumors. 

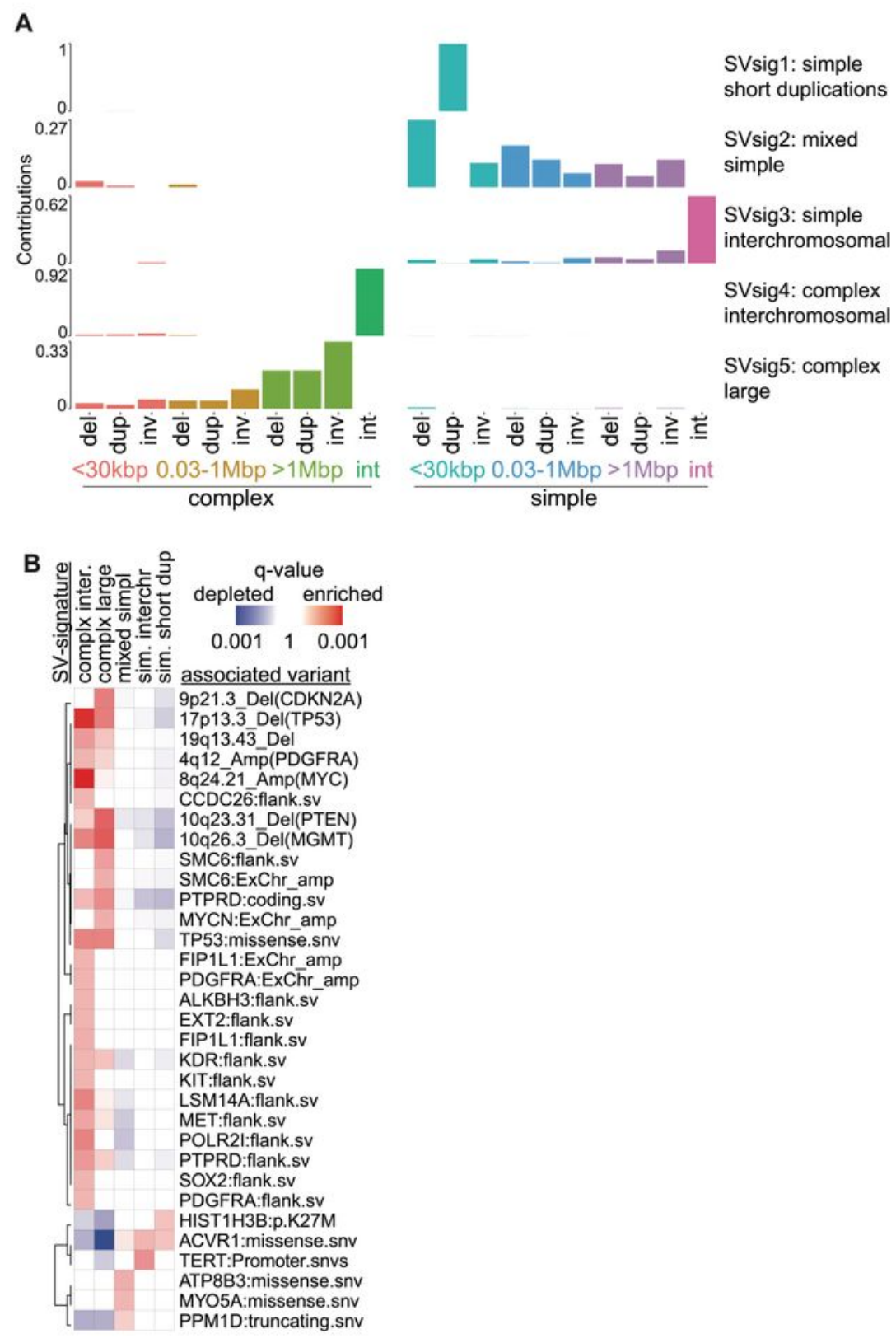

\section{Figure 4}

SV signatures in pHGG. (A) The horizontal axis indicates the size and type of SVs. Del stands for deletion, dup for duplication, inv for inversion, and int for interchromosomal rearrangement. The vertical axis indicates the fraction of SVs within each signature that are contributed by each SV type. (B) The statistical significance of positive (enriched) and negative (depleted) associations between each SV 
signature and of all recurrently altered somatic genetic alterations that are documented in the Cancer Gene Census53.
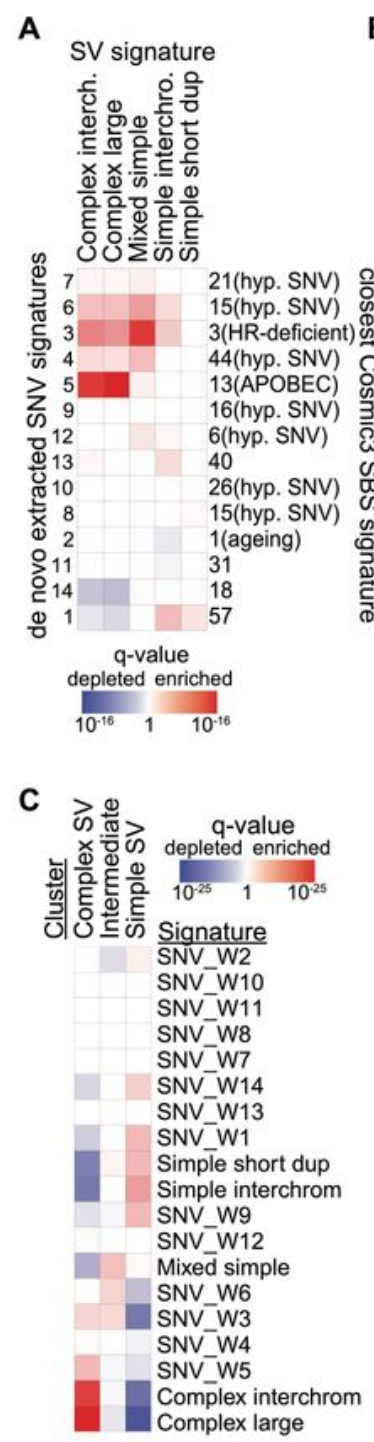

Complex SV Intermediate Simple SV

B Histone SNV

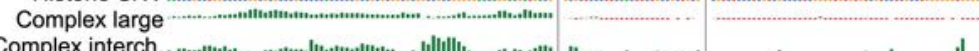

Simple interchro.

Simple short dup

Mixed simple

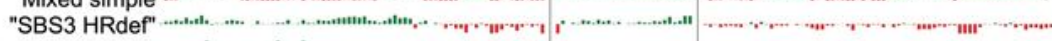

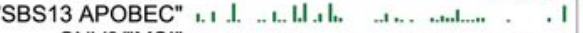

SNV9/"MSI"

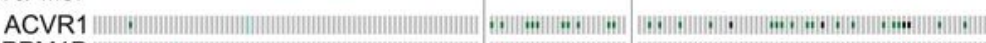

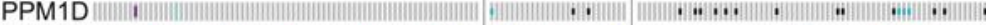

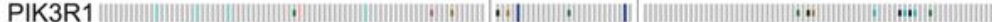

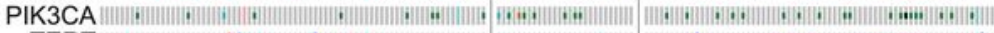

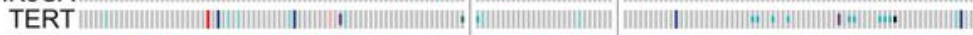

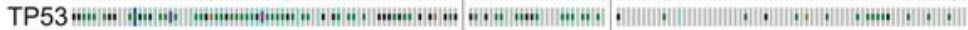

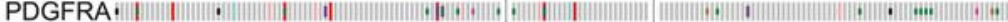

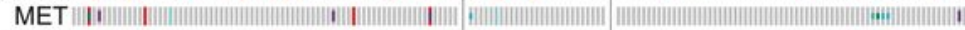
EGFR |

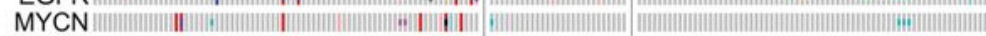
MYC

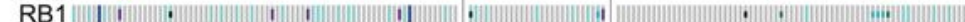
CDKN2A I PTEN :

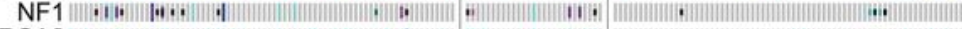

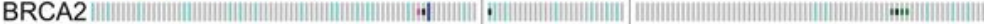

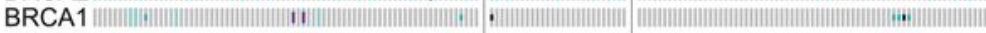
Histone SNV Genetic alteration

|H3.1K27M |H3.3K27M Missense mutation "Truncating mutation |H3.3G34R |WT | $\quad$ Promoter mutation | Other mutation inframe mutation (unknown significance) Coding SV $\mid \mathrm{CN}>10 \quad \| \mathrm{CN}>4.2$
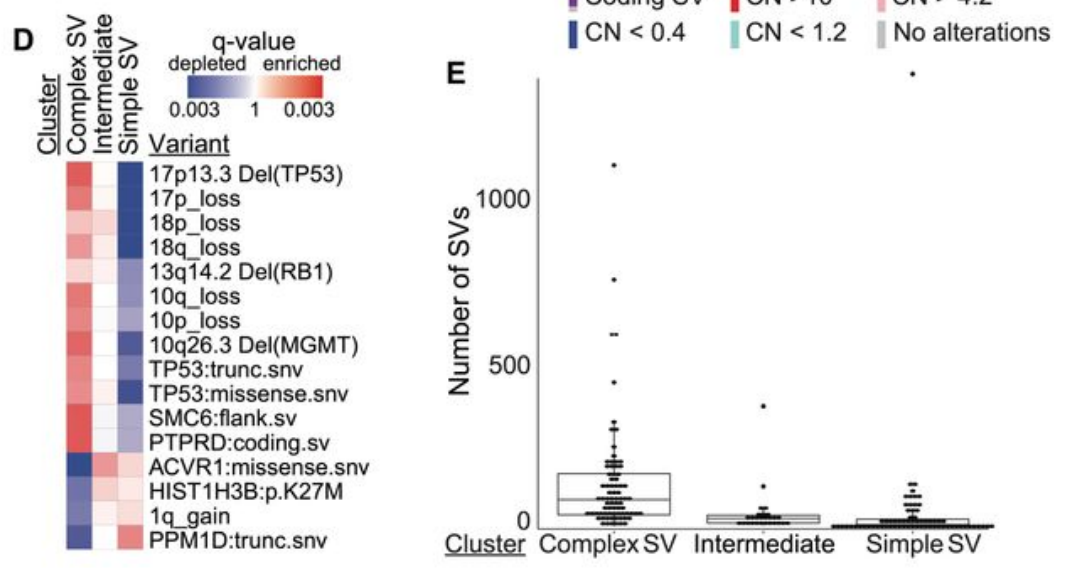

Cluster ComplexSV Intermediate SimplesV

Figure 5

Integrated SV and SNV signature analyses reveal three pHGG subgroups with distinct variant generating processes. (A) Correlations between SV and SNV signatures. Signature labels from this analysis are indicated on the left; the nearest COSMICv3 signatures are indicated on the right, with their proposed 
mechanisms in parentheses. Complex-SV signatures show a close correlation with APOBEC and homologous recombination deficiency SNV signatures (SBS3). q-values are based on Spearman rank correlations. (B) Consensus clustering of the normalized SNV and SV signature activities in each tumor sample (columns). Rows indicate signature activities (top) and potentially oncogenic variants (bottom). (C) Enrichment analysis for signature activities in each cluster from panel B. FDR q-values are based on Wilcoxon tests. (D) Significance of signature cluster associations for all variants with correlations reaching $\mathrm{q}<0.1$; q-values are based on Fisher's exact tests. Tumors in the complex-SV clusters are enriched for copy-number changes in cancer genes and SNVs in TP53, whereas simple-SV pHGGs tend to exhibit SNVs in different cancer genes. (E) Number of SVs per tumor in each cluster. All differences are significant to $\mathrm{q}<0.003$ by Wilcoxon tests. 
Complex SV sig Signature cluster ниіниніниниіні

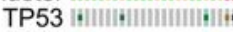

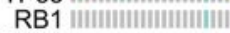
CDKN2A |||||||||||||||||||||||

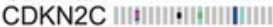
FAF1 แแแแแแแแแแ PPM1D I* *

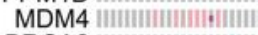

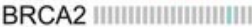

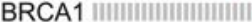

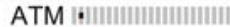

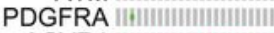

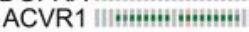

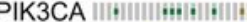

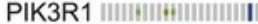

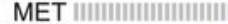

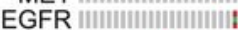

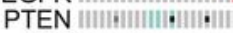

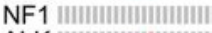

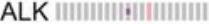

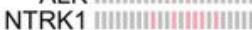

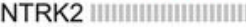

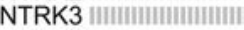

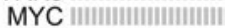
CCDC26 || แ||||||เ||||||||||| MYCN

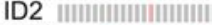

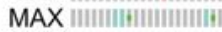

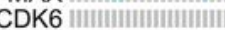

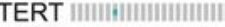

ASXL1 I

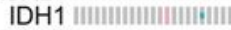

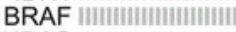

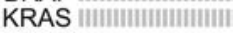

SETD2

KMT2D |||||||||||||||||||||||

KMT2C

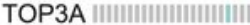

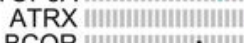

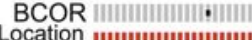
Signature cluster |Complex SV || Intem
| Simple SV |NA

B

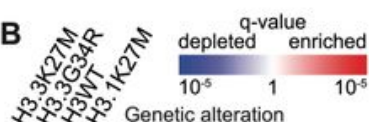

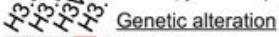
2p25.1 Amp 2p25.1 Amp(KIDINS220)

$2 p$ gain

2p gain

2p24.3 Amp(MYCN)

HIST1H3C missense.sn

$7 p$ gain

HIST1H3Bp.K27M

1q gain

FAF1 coding.sv

ACVR1 missense.snv

7p11.2 Amp(EGFR)

7p15.1 Amp

CDKN2B homde

CDKN2Ahomdel

7q21.2 Amp(CDK6)

ATRX truncating.snv

H3F3Amissense.snv

11 ploss

17p13.3 Del(TP53)

$17 p$ loss

8q24.21 Amp(CCDC26)

ASXL1 truncating.snv

KIT flank.sv

FIP1L1 flank.sv

H3F3Ap.K27M

TP53missense.snv

4q35.1 Del

TP53 truncating.snv |

H3.3K27M

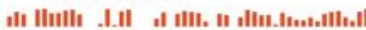
I" || 6 | . -

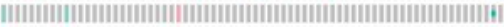

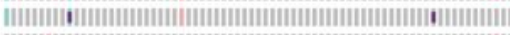

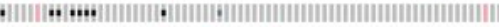

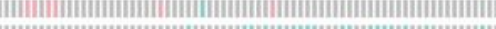

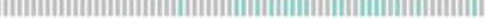

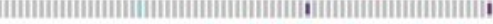

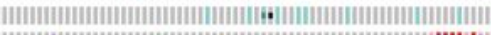

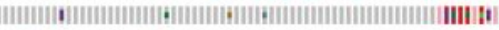

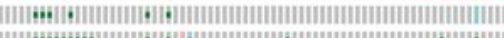

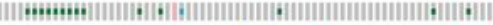

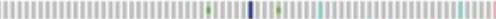

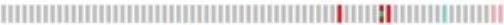

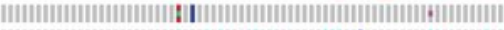

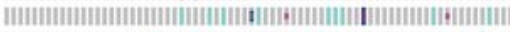

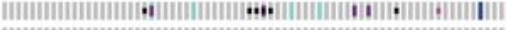

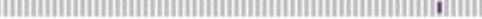

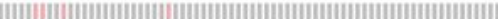

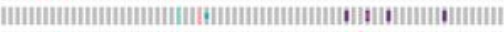

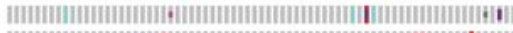

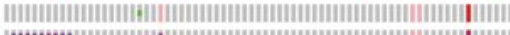
-

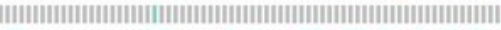
| (1).

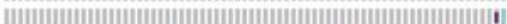

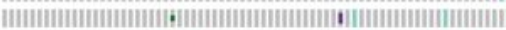

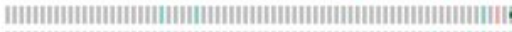

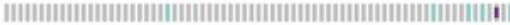

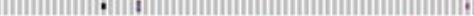

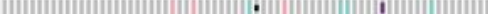
.

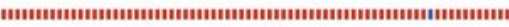

termediate Genetic alteration

- Missense mutation (putative driver)

| Missense mutation (unknown significance)

- Truncating mutation (putative driver)

| Truncating mutation (unknown significance)

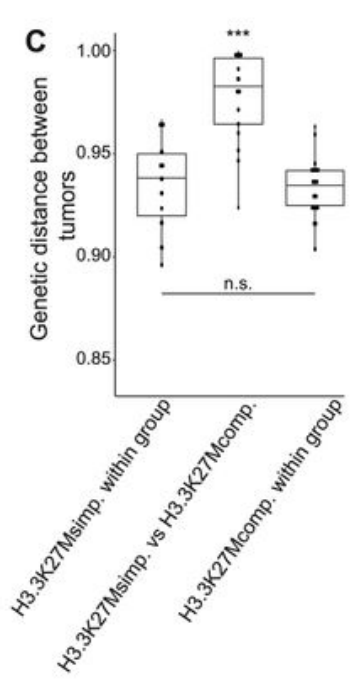

H3.3G34R

H3WT

Hyper

SNV

III IIIIIIII |

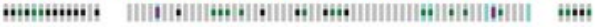

. IIIIIIIIII - ||

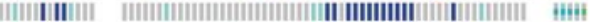

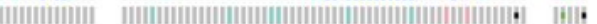

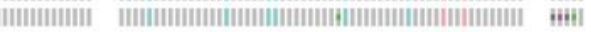

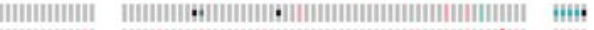

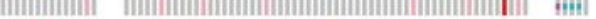

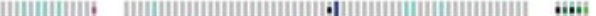

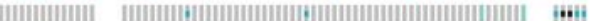

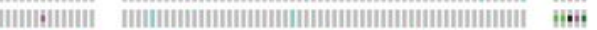

||

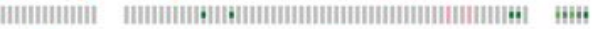

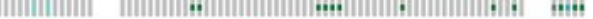

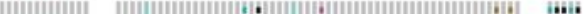

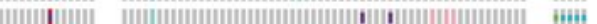

|І-

| IIII-

IIIIIIIIIII TIII,

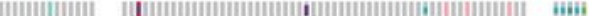

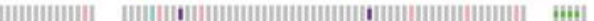

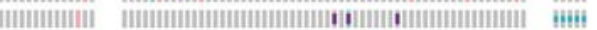

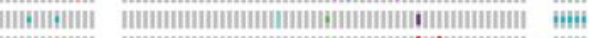

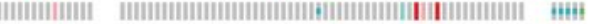

D.

แ

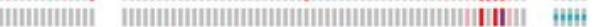

"

|

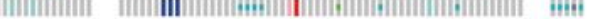

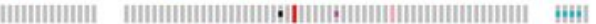

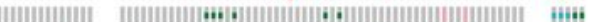

(IIเ,

| IIIIIIIIIII |

GH

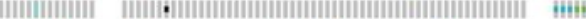

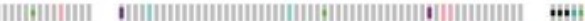

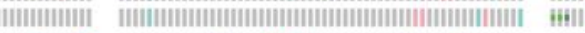

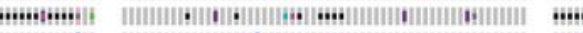

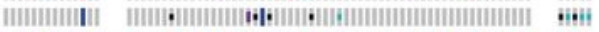

(1)

II Promoter mutation Other mutation Location

II Inframe mutation (unknown significance) \Hemispheric

II Coding SV | $\mathrm{CN}>10 \quad \| \mathrm{CN}>3.4 \quad$ |Midline

$\mathrm{CN}<0.4 \quad \mathrm{CN}<1.2 \quad$ No alterations

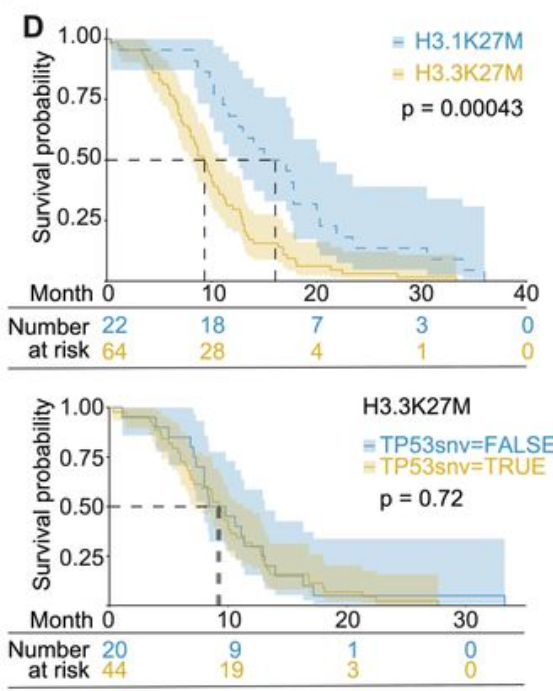

Figure 6

Overview of somatic variants and associated features within histone mutation-defined subgroups. (A) Comut plot of the 176/179 (98.3\%) tumors with somatic variants in at least one well-known oncogene.

Columns represent tumors, ordered within histone mutation-defined subgroups by hierarchical clustering of all potential driver variants. The top two rows show signature metadata. (B) Significance of histone subgroup associations for all variants with correlations reaching $\mathrm{q}<0.05$ (based on Fisher's exact tests) 
within any subgroup. (C) Jaccard distances based upon the variants in each tumor (vertical axis), for pairs of tumors within the H3.3K27M Simple-SV group (left column), within the H3.3K27M Complex-SV group (right column), or paired between these groups (middle column). H3.3K27M tumors were considered to be in the Complex-SV group if Complex-SV signatures comprised more than $20 \%$ of its SV signature activity. ${ }^{* \star *}$ denotes $\mathrm{q}<6 \times 10-8$ relative to both other columns by a Wilcoxon test. (D) KaplanMaier plot indicating overall survival for (top) H3.1K27M and H3.3K27M DMGs and (bottom) H3.3K27M DMG with and without TP53 SNVs. p-values are from log-rank tests.

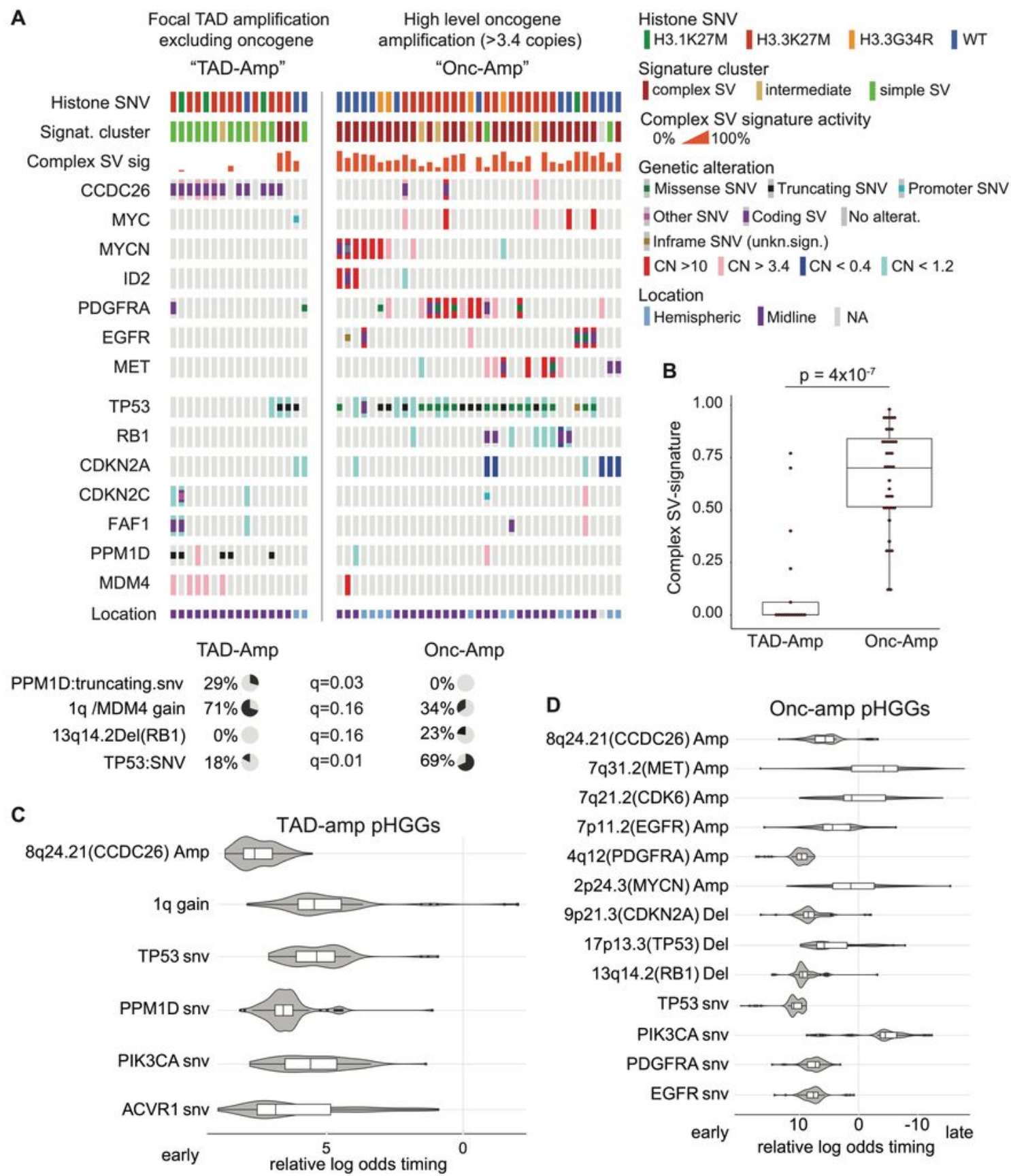




\section{Figure 7}

Context of the significantly recurrent SVs. (SRSVs) (A) Co-mut plot for TAD-amp and Onc-amp SRSVs. TAD-amp SRSVs generate focal amplifications in the TAD of an oncogene without amplifying the protein coding sequence; Onc-amp SRSVs generate high-level ( $C N>3.4)$ amplifications or fusions of the coding sequence. The top three rows show associated metadata. The next seven rows indicate the genes affected by the SRSVs. The bottom seven rows show genes in DNA damage response pathways. Significant associations with the two groups are illustrated with pie charts below the plot, based on Fisher's exact tests. (B) TAD-amp pHGGs show significantly lower combined Complex-SV signature activity than Onc-amp pHGGs ( $p=4 \times 10-7$, Wilcoxon). (C-D) Timing analysis of somatic variant acquisition in TAD-amp (C) and Onc-amp (D) pHGGs based on a Bradley-Terry model. The horizontal axis shows the log odds of the variant being an early event. The distributions indicate the results of 100 random subsamples of the data. Only variants in the SRSV-affected genes and pathways (growth factor and MYC signaling) and in DNA damage response genes altered in more than two samples are shown. 

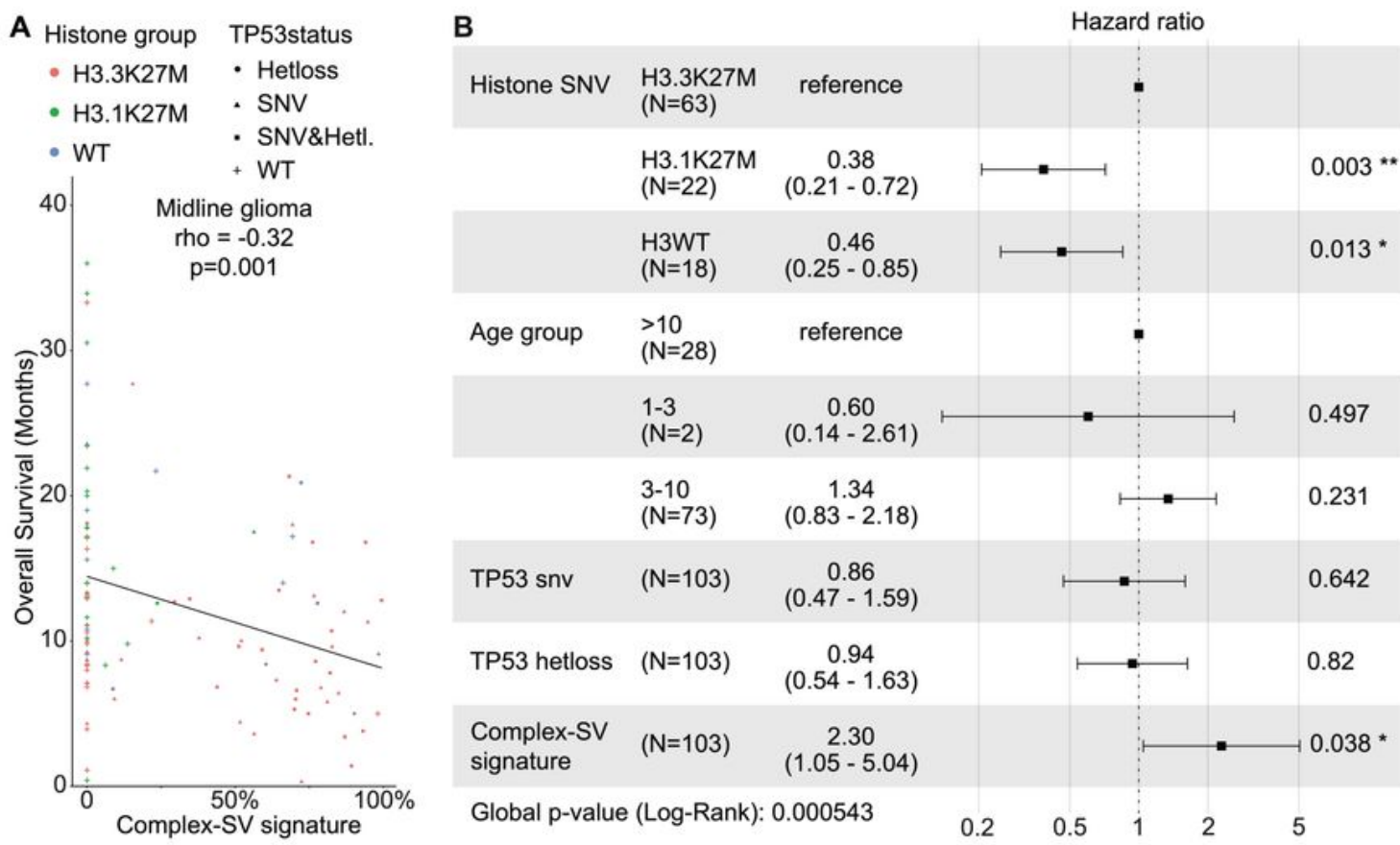

C

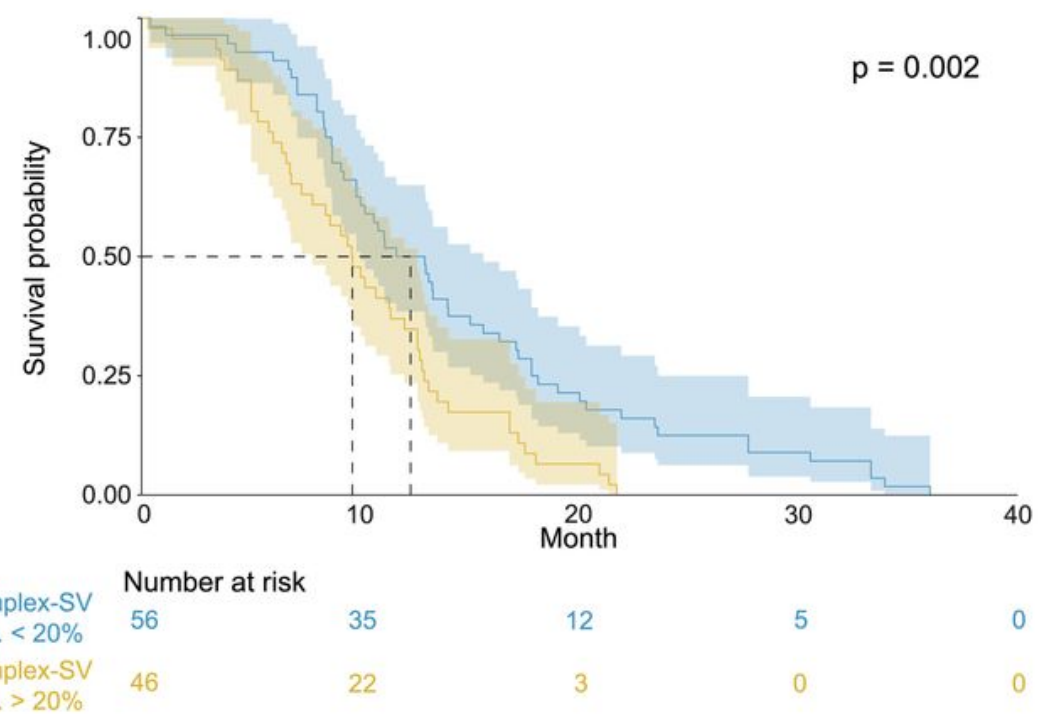

\section{Figure 8}

Association between the complex-SV signature and overall survival in midline gliomas. (A) Associations between the fraction of SV signature activity attributed to Complex-SV signatures and overall survival for all midline gliomas ( $n=103$; rho $=-0.32$ and $p=0.001$, Spearman correlation). (B) Cox proportional hazards analysis incorporating histone group, age, TP53 status and the combined Complex-SV signature. (C) Kaplan-Maier plot of a univariate analysis of the association between the Complex-SV signature and 
overall survival, in which tumors were classified as Complex-SV if Complex-SV signatures contributed more than $20 \%$ of all SV signature activity. The number of patients in each group is indicated below the plot. The p-value represents a log-rank test.

\section{Supplementary Files}

This is a list of supplementary files associated with this preprint. Click to download.

- supp.table1metaDatabySample.txt

- supp.table2SVmafs.txt

- supp.table3RSBs.txt

- supp.table4SVsigsVarCorr.xlsx

- supp.table5VariantCombinationMatrix.txt

- supp.table6LuciferaseReporterPrimers.xIsx

- 20210403pHGGsuppfigures.pdf

- 20210403bExt.DataFigures.pdf 